\title{
The Pulse of Asia 2018 Kyoto
}

May 19-20, 2018, Kyoto, Japan

Guest Editor

Kazuomi Kario, Japan

\section{Contents}

41 Abstracts SL1-SL2

42 Abstracts JS1-01-JS1-06

45 Abstracts SY1-01-SY4-03

53 Abstracts SS-1-01-SS-1-03

54 Abstracts LS1-01-LS2-03

57 Abstracts PL01-01-PL-04

60 Abstracts YIA-01-YIA-04

63 Abstracts O-01-O-05

67 Abstracts MP-01- MP-30

92 Abstracts P-01-P-08

KARGER ER 


\section{Welcome Letter}

\section{Pulse of Asia 2018 in Kyoto}

Systemic hemodynamic atherothrombotic syndrome

-Vicious cycle of hemodynamic variability and vascular disease-

We cordially welcome all of you to attend the scientific meeting of the Pulse of Asia 2018, to be held on 19-20 May at the Miyako Messe Center, Kyoto, Japan.

The main theme of the POA2018 in Kyoto is "systemic hemodynamic atherothrombotic syndrome (SHATS)". This is a concept of vicious cycle of hemodynamic variability and vascular property on organ damage and cardiovascular events. The program will consist of sessions of hot topics of pathophysiology-based clinical evaluations of vascular properties and blood pressure variability. As the facilitator and/or the modulator of the SHATS, aging kidney and inflammation, regional differences in healthcare environmental factors, and renal denervation will be focused. In this meeting, we clarify the concept of HATS (Hemodynamic ArTeriosclerotic Syndrome) in a narrower sense of previous SHATS concept, by separating the well-known atherothrombotic mechanism of cardiovascular disease from the increasingly important arteriosclerotic mechanism.

In addition, we will have the joint sessions with the Hypertension Forum, spring meeting of Japanese Society of Hypertension (PresidentMasaaki Ito), and the HOPE Asia Network meeting, an Asian activity of World Hypertension League. The HOPE Asia network is aiming to improve the management of hypertension and organ protection for "zero" cardiovascular event in Asia, in consideration of Asian characteristics.

The Pulse of Asia 2018 promises to provide the up-date information of the cardiovascular research on the artery and blood pressure, and the warm "agora" for the fruitful discussion on the Asian characteristics of vascular health.

Welcome to the Pulse of Asia 2018 in Kyoto!

Kazuomi Kario

Chairman, POA Annual Meeting 2018

Jiguang Wang

President, The Pulse of Asia 
Abstracts: The Pulse of Asia 2018 Kyoto

\section{Hemodynamic ArTeriosclerotic Syndrome (HATS) - A Vicious Cycle of Hemodynamic Stress and Vascular Disease}

\section{Kazuomi Kario, MD, $P h D^{1,2}$}

${ }^{1}$ Division of Cardiovascular Medicine, Department of Medicine, Jichi Medical University School of Medicine, and Jichi Medical University Center of Excellence, Cardiovascular Research and Development (JCARD), Tochigi, Japan; ${ }^{2}$ Hypertension Cardiovascular Outcome Prevention and Evidence in Asia (HOPE Asia) Network, Tochigi, Japan

There are two common types of arterial disease: endothelial damage-initiated atherosclerosis resulting in advanced atherosclerotic plaque, and arteriosclerosis with advanced arterial stiffness of large arteries, which is linked to small artery remodeling. The former is predominantly promoted by metabolic risk factors, whereas the latter is most closely linked to aging and hypertension. The definitions of SHATS (Systemic Hemodynamic ATherothrombotic Syndrome), a new concept of the synergistic vicious cycle of blood pressure (BP) variability and vascular disease, have included both types of vascular disease. However, here, we clarify the concept of HATS (Hemodynamic ArTeriosclerotic Syndrome) in a narrower sense of previous SHATS concept. HATS refers to an accelerated vicious cycle of hemodynamic stress (BP variability/surge) and arteriosclerosis (arterial stiffness), resulting in target organ damage and cardiovascular events.

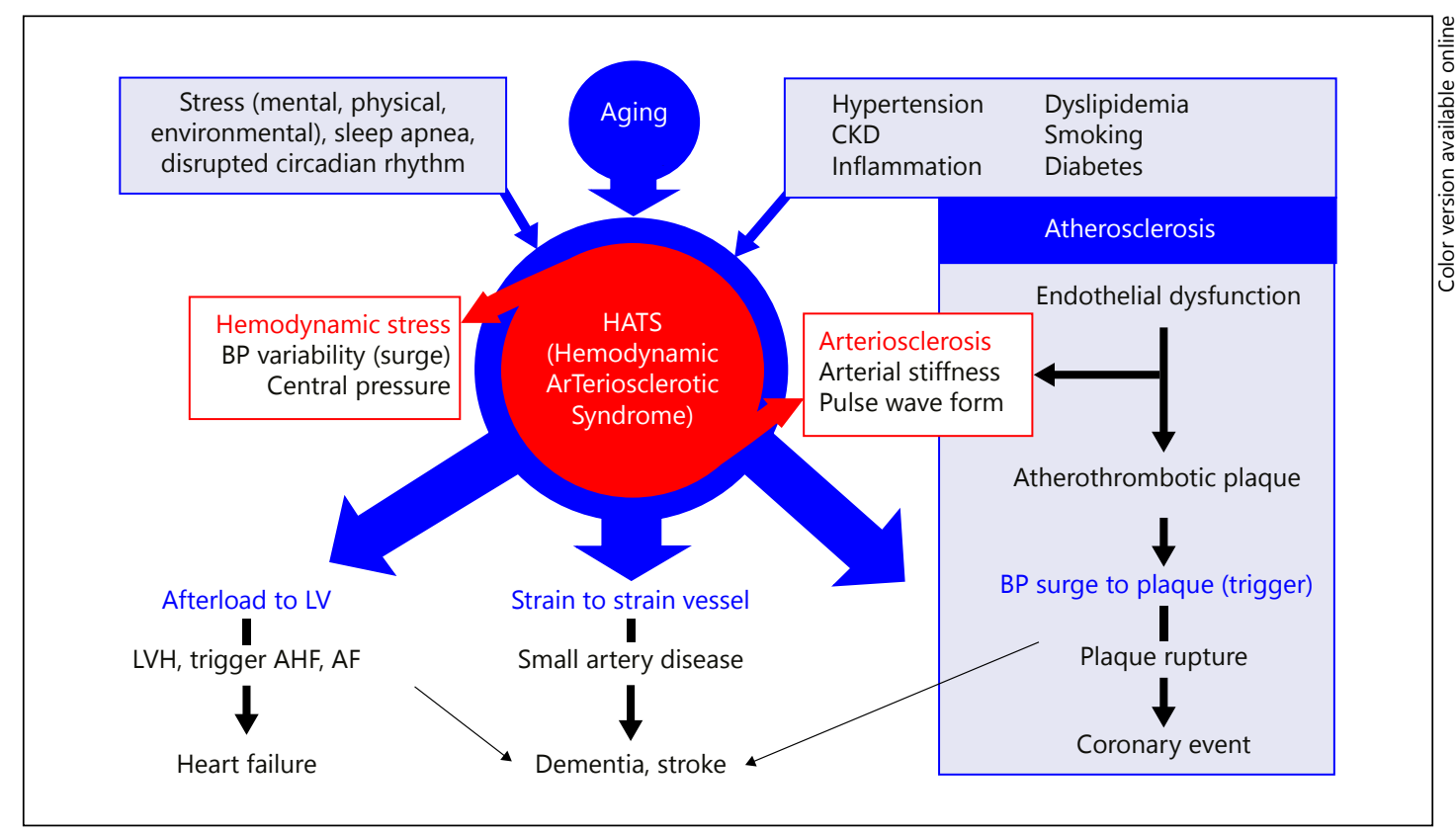

HATS and Cardiovascular Disease

Source: Kario K. Hemodynamic ArTeriosclerotic Syndrome (HATS) - A vicious cycle of hemodynamic stress and vascular disease. J Clin Hypertens (Greenwich). 2018, in press. 


\section{Program}

\section{POA 2018 Program}

\section{May (Sat), 2018}

\begin{tabular}{|c|c|c|c|}
\hline \multicolumn{4}{|c|}{$\begin{array}{l}\text { Oral Session } \\
\text { Exhibition Hall 1-B, B1, Miyakomesse }\end{array}$} \\
\hline $8: 20-8: 30$ & \multicolumn{2}{|c|}{ Opening Remarks } & Kazuomi Kario (Japan) \\
\hline 8:30-10:40 & \multicolumn{2}{|c|}{ Conncept and New Evidence of Vascular Biomarker } & $\begin{array}{l}\text { Chair: } \\
\text { Michael O’Rourke (Australia) } \\
\text { Yusuke Ohya (Japan) } \\
\text { Hirofumi Tomiyama (Japan) }\end{array}$ \\
\hline $8: 30$ & SY1-01 & Basic Concepts and New Evidence of Vascular Biomarkers & Alberto Avolio (Australia) \\
\hline $8: 50$ & SY1-02 & Novel Arterial Biomarkers & Julio A Chirinos (USA) \\
\hline 9:10 & SY1-03 & $\begin{array}{l}\text { Central Blood Pressure and Pressure Wave Reflection in Early } \\
\text { Stages of Cardiovascular Abnormalities }\end{array}$ & Hirofumi Tomiyama (Japan) \\
\hline $9: 25$ & SY1-04 & Aortic Stiffness and Hemodynamics in Chronic Kidney Disease & Junichiro Hashimoto (Japan) \\
\hline $9: 40$ & SY1-05 & FMD & Yukihito Higashi (Japan) \\
\hline 9:55 & SY1-06 & The Methodology, Clinical Use and Evidence of CAVI & Tomoyuki Kabutoya (Japan) \\
\hline $10: 10$ & SY1-07 & $\begin{array}{l}\text { Recent Evidence on Brachial-Ankle PWV-Why it is Better Than } \\
\text { Initially Expected? }\end{array}$ & Masanori Munakata (Japan) \\
\hline $10: 25$ & SY1-08 & $\begin{array}{l}\text { Ankle-Brachial Index (ABI) is a Marker for Both } \\
\text { Atherosclerosis and Arteriosclerosis }\end{array}$ & Akio Ishida (Japan) \\
\hline $10: 40-12: 10$ & $\begin{array}{l}\text { JSH-POA } \\
\text { Systemic }\end{array}$ & $\begin{array}{l}\text { Joint Session: } \\
\text { Hemodynamic Atherothrombotic Syndrome }\end{array}$ & $\begin{array}{l}\text { Chair: } \\
\text { Alberto Avolio (Australia), } \\
\text { Hiromi Rakugi (Japan) } \\
\text { Julio A. Chirinos (USA) } \\
\text { Commentator: } \\
\text { James Sharman (Australia), } \\
\text { Michael O'Rourke (Australia) } \\
\text { Jeong Bae Park (Korea) }\end{array}$ \\
\hline $10: 40$ & JS1-01 & WHAT IS SHATS? & Kazuomi Kario (Japan) \\
\hline 10:55 & JS1-02 & Strain Vessel Theory: An Evolutional Point of View & Sadayoshi Ito (Japan) \\
\hline $11: 10$ & JS1-03 & Blood Pressure Variability and Vascular Disease & Ji-Guang Wang (China) \\
\hline $11: 25$ & JS1-04 & Blood Pressure Variability and Organ Damage & Mitsuru Ohishi (Japan) \\
\hline $11: 40$ & JS1-05 & Exercise $=$ Severe Hemodynamic Stress = Increased Risk $?$ & Bo Fernhall (USA) \\
\hline 11:55 & JS1-06 & $\begin{array}{l}\text { Hypertension is a System Disruption of Dynamic Blood } \\
\text { Pressure Regulation Mediated by Brain }\end{array}$ & Takuya Kishi (Japan) \\
\hline 12:10-13:00 & Luncheo & n Seminar (Sponsored by OMRON HEALTHCARE Co., Ltd) & $\begin{array}{l}\text { Chair: } \\
\text { Masaaki Ito (Japan) } \\
\text { Naoyuki Hasebe (Japan) }\end{array}$ \\
\hline $12: 10$ & LS1-01 & New Approaches for Evaluating and Managing Hypertension & Michael Weber (USA) \\
\hline $12: 35$ & LS1-02 & $\begin{array}{l}\text { The Steno-Stiffness Chart Deriving from Simultaneous } \\
\text { Measurement of Ankle Brachial Pressure Index (ABI) and } \\
\text { Brachial-Ankle Pulse Wave Velocity (baPWV) for the Wide } \\
\text { Range Risk Stratification }\end{array}$ & Akira Yamashina (Japan) \\
\hline
\end{tabular}


Abstracts: The Pulse of Asia 2018 Kyoto

\begin{tabular}{|c|c|c|c|}
\hline $13: 10-14: 00$ & \multicolumn{2}{|c|}{ BP Variability I } & $\begin{array}{l}\text { Chair: } \\
\text { James Sharman (Australia) } \\
\text { Satoshi Hoshide (Japan) }\end{array}$ \\
\hline $13: 25$ & SY2-02 & $\begin{array}{l}\text { Home Blood Pressure Variability Based on the Routine } \\
\text { Self-Measurement }\end{array}$ & Kei Asayama (Japan) \\
\hline $13: 40$ & SY2-03 & $\begin{array}{l}\text { Prognostic Significance of Home BP Variability on Diabetic } \\
\text { Nephropathy: KAMOGAWA-HBP Study }\end{array}$ & Emi Ushigome (Japan) \\
\hline $14: 00-14: 50$ & $\mathrm{ABPM}(\mathrm{S}$ & ponsored by A\&D Company Limited) & $\begin{array}{l}\text { Chair: } \\
\text { Jeong Bae Park (Korea) } \\
\text { Takayoshi Ohkubo (Japan) }\end{array}$ \\
\hline $14: 15$ & SS-1-02 & ABPM in China & Ji-Guang Wang (China) \\
\hline $14: 30$ & SS-1-03 & $\begin{array}{l}\text { Multicenter Ambulatory Blood Pressure Monitoring Registry } \\
\text { in Korea }\end{array}$ & Jinho Shin (Korea) \\
\hline \multirow[t]{2}{*}{$15: 00-15: 30$} & \multicolumn{2}{|c|}{ Special Lecture I } & $\begin{array}{l}\text { Chair: } \\
\text { Der-Cherng Tarng (Taiwan) } \\
\text { Kouichi Tamura (Japan) }\end{array}$ \\
\hline & SL1 & Ageing Kidney and Inflammation & Makoto Kuro-o (Japan) \\
\hline $15: 40-16: 10$ & \multicolumn{2}{|c|}{ Special Lecture II } & $\begin{array}{l}\text { Chair: } \\
\text { Hiroshi Itoh (Japan) } \\
\text { Hirofumi Tomiyama (Japan) }\end{array}$ \\
\hline $16: 20$ & SY3-01 & Seasonal Variation in Blood Pressure & Ji-Guang Wang (China) \\
\hline $16: 35$ & SY3-02 & $\begin{array}{l}\text { Prognostic Significance of Morning Hypertension Associated } \\
\text { with Sustained Nocturnal Hypertension }\end{array}$ & Sung-Ha Park (Korea) \\
\hline $16: 50$ & SY3-03 & $\begin{array}{l}\text { What Is the Contribution of Blood Pressure Measurement } \\
\text { Accuracy in Systemic Hemodynamic Atherothrombotic } \\
\text { Syndrome? }\end{array}$ & James Sharman (Australia) \\
\hline $17: 30-18: 30$ & Renal De & nervation Up-to-Date (TBD) & $\begin{array}{l}\text { Chair: } \\
\text { Yoshitaka Hirooka (Japan) } \\
\text { Michael Weber (USA) } \\
\text { Commentator: } \\
\text { Akira Nishiyama (Japan) } \\
\text { Sung-Ha Park (Korea) }\end{array}$ \\
\hline $17: 30$ & SY4-01 & New Evidence and Perspective in RDN & Raymond R. Townsend (USA) \\
\hline $18: 00$ & SY4-02 & The Procedure of Renal Denervation for Resistant Hypertension & Yukako Ogoyama (Japan) \\
\hline $18: 10$ & SY4-03 & Perspectives in Renal Denervation in Asia & Kazuomi Kario (Japan) \\
\hline $18: 30-19: 00$ & & Board Meeting & \\
\hline \multicolumn{4}{|c|}{$\begin{array}{l}\text { Poster Session } \\
\text { Exhibition Hall 2-CD, 1F, Miyakomesse }\end{array}$} \\
\hline \multirow[t]{2}{*}{$15: 00-16: 00$} & \multicolumn{2}{|c|}{ P1 Pathology \& Experimental } & $\begin{array}{l}\text { Chair: } \\
\text { Michael O’Rourke (Australia) } \\
\text { Ken Kono (Japan) }\end{array}$ \\
\hline & MP-01 & Adhesion Molecules as a Novel Biomarker for Vascular Ageing & Sukrat Sinha (India) \\
\hline
\end{tabular}


Abstracts: The Pulse of Asia 2018 Kyoto

\begin{tabular}{|c|c|c|}
\hline MP-02 & $\begin{array}{l}\text { A High Normal Ankle-Branchial Index Is Associated with Renal } \\
\text { Small Artery Intimal Thickness in Chronic Kidney Disease }\end{array}$ & Ryo Zamami (Japan) \\
\hline MP-03 & $\begin{array}{l}\text { The Effect of Cardiac Valve Calcification on Long term Clinical } \\
\text { Prognosis in Patients with Peripheral Vascular Disease } \\
\text { Undergoing Percutaneous Transluminal Angioplasty }\end{array}$ & Ji Young Park (Korea) \\
\hline MP-04 & $\begin{array}{l}\text { Association of Pulse Wave Velocity with Single Nucleotide } \\
\text { Polymorphisms Related to Parathyroid Hormone }\end{array}$ & Yi-Bang Cheng (China) \\
\hline MP-05 & $\begin{array}{l}\text { The Differential Impacts of Dialysis Vintage and Age on } \\
\text { Hemodynamics and Autonomic Nerve Activity in Patients } \\
\text { Under Hemodialysis }\end{array}$ & Han-Kuei Wu (Taiwan) \\
\hline MP-06 & $\begin{array}{l}\text { Lower Urinary Tract Symptoms in Men as an Early Phenotype } \\
\text { of Endothelial Dysfunction }\end{array}$ & Shogo Matsui (Japan) \\
\hline MP-07 & $\begin{array}{l}\text { Carotid Artery Atherosclerosis Is Closely Associated with } \\
\text { Reduced Membrane Fluidity of Red Blood Cells in } \\
\text { Normotensive and Hypertensive Subjects: An Electron Spin } \\
\text { Resonance (ESR) Study }\end{array}$ & Kazushi Tsuda (Japan) \\
\hline P2 Epid & miology \& Clinical Studies & $\begin{array}{l}\text { Chair: } \\
\text { Tomoyuki Kabutoya (Japan) } \\
\text { Tatsuya Maruhashi (Japan) }\end{array}$ \\
\hline SP & $\begin{array}{l}\text { Best Presenter of Korean Vascular Research Working Group } \\
\text { Special Presentation: Prevention of Vascular Stenosis Using an } \\
\text { External Wall Wrapping Device }\end{array}$ & Jeong-Kee Yoon (Korea) \\
\hline MP-08 & $\begin{array}{l}\text { Different Impact of Nondipping Blood Pressure Pattern for } \\
\text { Cardiovascular Outcome Between the Population with and } \\
\text { Without Carotid Atherosclerosis: The Japan Morning } \\
\text { Surge-Home Blood Pressure Study }\end{array}$ & Praew Kotruchin (Japan) \\
\hline MP-09 & $\begin{array}{l}\text { Nocturnal Pulse Pressure Was Associated with Adverse } \\
\text { Outcomes in Heart Failure with Preserved Ejection Fraction of } \\
\text { Clinical Scenario } 1\end{array}$ & Takahiro Komori (Japan) \\
\hline MP-10 & $\begin{array}{l}\text { Developing and Validating a New Precise Risk-Prediction } \\
\text { Model for New-OnsetHypertension: The Jichi Genki } \\
\text { Hypertension Prediction Model (JG Model) }\end{array}$ & Hiroshi Kanegae (Japan) \\
\hline MP-11 & $\begin{array}{l}\text { Prevalence and Characteristics of Exaggerated Morning Surge } \\
\text { and Morning Hypertension in Chinese patients: China } \\
\text { Ambulatory and Home Blood Pressure Registry (ABPR) }\end{array}$ & Qian-Hui Guo (China) \\
\hline MP-12 & $\begin{array}{l}\text { Metabolic Syndrome Status Over } 2 \text { Years Predicts Incident } \\
\text { Chronic Kidney Disease: A 10-Year Prospective Cohort Study }\end{array}$ & Jang Young Kim (Korea) \\
\hline MP-13 & $\begin{array}{l}\text { Gender Differences in Five years Long term Clinical Outcomes } \\
\text { of Coronary Artery Spasm in Korean Population using } \\
\text { Propensity Matching Analysis }\end{array}$ & Ji Young Park (Korea) \\
\hline MP-14 & $\begin{array}{l}\text { Relationship Between an Increased Urinary Albumin Excretion } \\
\text { and Abnormal Electrocardiogram in the General Population }\end{array}$ & Satoshi Konno (Japan) \\
\hline MP-15 & $\begin{array}{l}\text { A Comparison of Brachial Artery Diameter with Flow-Mediated } \\
\text { Vasodilation as Vascular Marker for Cardiovascular Risk } \\
\text { Assessment: FMD-J Study }\end{array}$ & Tatsuya Maruhashi (Japan) \\
\hline \multicolumn{3}{|c|}{ Poster (non-chaired) } \\
\hline P-01 & $\begin{array}{l}\text { Determinants of Inaccuracy of Validated Blood Pressure } \\
\text { Monitors on Standardized Conditions }\end{array}$ & Lei Lei (China) \\
\hline P-02 & $\begin{array}{l}\text { Effects of the Valsartan/Amlodipine Combination and the } \\
\text { Nifedipine GITS Monotherapy on Ambulatory Blood Pressure, } \\
\text { Arterial Stiffness and Wave Reflection in Patients Inadequately } \\
\text { Controlled with Monotherapy }\end{array}$ & Shaokun Xu (China) \\
\hline
\end{tabular}


P-03 Role of Bisphenol a Exposure During Gestation and Lactation Arun Gandhi (India) on the Emotional Behavior and the Content of Brain

Neurotransmitters in Rat Offspring

19:00 Welcome Party

(with the 7th Annual Scientific Forum of Clinical Hypertension of the JSH)

20 May (Sun), 2018

Oral Session

Exhibition Hall 1-B, B1, Miyakomesse

7:30-9:00 HOPE Asia Network Business Meeting

9:00-9:30 HOPE Asia / WHL - Publication Achievement Chair:

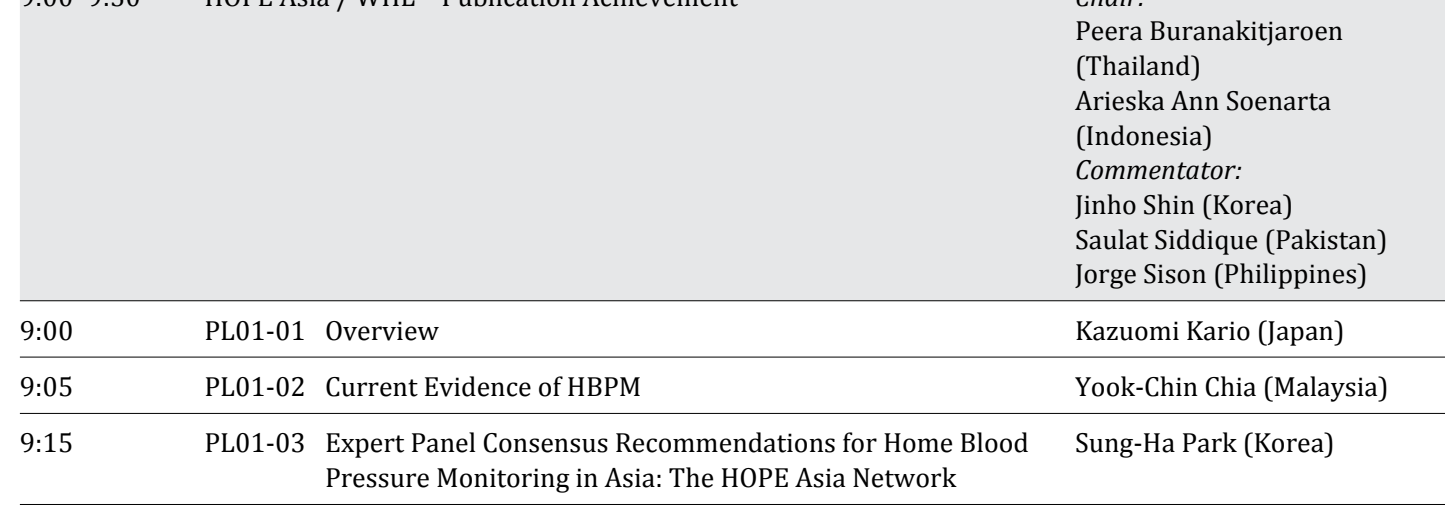

9:25 PL01-04 Morning Hypertension Ji-Guang Wang (China)

9:30-10:00 HOPE Asia / WHL - Round Table Discussion on BP Variability and Morning Chair:

Surge Paper Ji-Guang Wang (China)

Jam Chin Tay (Singapore)

Commentator:

Michael Weber (USA)

PL-02 Blood Pressure Variability and Morning BP Surge Guru Prasad Sogunuru (India)

10:00-10:30 WHL - Management of Hypertension in Globe Chair:

Kazuomi Kario (Japan)

Yuda Turana (Indonesia)

PL-03 Worldwide Cardiovascular Risk and Hypertension

Michael Weber (USA)

A Partnership of HOPE Asia and the World Hypertension

League (WHL)

\begin{tabular}{cl} 
10:35-10:55 AsiaBP@Home Study & \\
\hline PL-04 & $\begin{array}{l}\text { The First Report from a Study to Define the Distribution of } \\
\text { Type of Hypertension in Asia by Blood Pressure Monitoring at } \\
\text { Home -Asia BP@Home- }\end{array}$
\end{tabular}

\begin{tabular}{|c|c|c|c|}
\hline $11: 00-11: 40$ & YIA Ora & Session & Chair: \\
\hline $11: 00$ & YIA-01 & $\begin{array}{l}\text { Impact of Indoor Temperature on Morning Home Blood } \\
\text { Pressure in Winter: Baseline Data from a Non-Randomized } \\
\text { Controlled Trial in Japan }\end{array}$ & Wataru Umishio (Japan) \\
\hline $11: 10$ & YIA-02 & $\begin{array}{l}\text { Association of Pulsatile Hemodynamics and Cognitive } \\
\text { Dysfunction in Middle-Aged and Older Community Subjects: } \\
\text { Results from the Longitudinal Aging Study of Taipei (LAST) }\end{array}$ & Chen-Hua Lin (Taiwan) \\
\hline
\end{tabular}

Type of Hypertension in Asia by Blood Pressure Monitoring at 
Abstracts: The Pulse of Asia 2018 Kyoto

\begin{tabular}{|c|c|c|c|}
\hline $11: 20$ & YIA-03 & $\begin{array}{l}\text { The Non-Dipper Blood Pressure Pattern Is an Independent } \\
\text { Predictor of Cardiovascular Outcomes in Patients with } \\
\text { Sleep-Disordered Breathing: The J-HOP Study }\end{array}$ & Hiroyuki Mizuno (Japan) \\
\hline $11: 30$ & YIA-04 & $\begin{array}{l}\text { The Association of Intracranial Arterial Stenosis with Home } \\
\text { Blood Pressure Level and Variability }\end{array}$ & Dongyan Zhang (China) \\
\hline $11: 40-12: 20$ & \multicolumn{2}{|c|}{ Special Oral Presentations } & $\begin{array}{l}\text { Chair: } \\
\text { Masataka Sata (Japan) } \\
\text { Michael O’Rourke (Australia) }\end{array}$ \\
\hline $11: 40$ & $0-01$ & $\begin{array}{l}\text { Hyperuricemia and Inflammation in the Increase in Arterial } \\
\text { Stiffness and Development of Hypertension }\end{array}$ & Masatsune Fujii (Japan) \\
\hline $11: 48$ & $0-02$ & $\begin{array}{l}\text { Ascending to Descending Aortic Pulse Pressure Amplification } \\
\text { in Children - Normal Aorta and Surgically Corrected Aorta }\end{array}$ & Tomoaki Murakami (Japan) \\
\hline $11: 56$ & $0-03$ & $\begin{array}{l}\text { Which Is More Correlated with Hypertensive Organ Damage, } \\
\text { Sleep Blood Pressure Assessed by Self-Measured at Home or } \\
\text { Ambulatory Blood Pressure Monitoring?: The Japan Morning } \\
\text { Surge-Home Blood Pressure (J-HOP) Study }\end{array}$ & Sirisawat Wanthong (Japan) \\
\hline $12: 04$ & $0-04$ & $\begin{array}{l}\text { Effects of a SGLT2 Inhibitor on Blood Pressure and } \\
\text { Sympathetic Nervous Activity in Salt-Treated CKD Rats }\end{array}$ & Ningning Wan (Japan) \\
\hline $12: 12$ & $0-05$ & $\begin{array}{l}\text { Effect of Mean and Pulse Pressure on Fracture of } \\
\text { Atherosclerotic Plaques }\end{array}$ & Alberto Avolio (Australia) \\
\hline $12: 30-13: 30$ & LS: CAVI & (Sponsored by Fukuda Denshi Co., Ltd) & $\begin{array}{l}\text { Chair: } \\
\text { Koichi Node (Japan) } \\
\text { Tohru Minamino (Japan) }\end{array}$ \\
\hline $12: 30$ & LS2-01 & $\begin{array}{l}\text { The Clinical Evidence of CAVI and the Comparison of } \\
\text { Reference Value in CAVI and baPWV in Coupling Registry }\end{array}$ & Tomoyuki Kabutoya (Japan) \\
\hline $12: 45$ & LS2-02 & $\begin{array}{l}\text { Evaluating the Benefits of Using CAVI as a Predictor of } \\
\text { Cardiovascular Events in High-Risk Patients in Japan (CAVI-J) }\end{array}$ & Toru Miyoshi (Japan) \\
\hline $13: 00$ & LS2-03 & Arterial Stiffness, CKD, CVD & Raymond R. Townsend (USA) \\
\hline \multirow[t]{5}{*}{$13: 30-14: 00$} & \multicolumn{2}{|c|}{ POA 2018 Awards and Closing Remarks } & $\begin{array}{l}\text { Chair: } \\
\text { Kazuomi Kario (Japan) } \\
\text { Ji-Guang Wang (China) }\end{array}$ \\
\hline & \multicolumn{2}{|r|}{ POA 2018 Award } & $\begin{array}{l}\text { Winner: } \\
\text { Kazuyuki Shimada (Japan) }\end{array}$ \\
\hline & \multicolumn{2}{|r|}{ Excellent Presentation Award } & \\
\hline & \multicolumn{2}{|r|}{ Introduction of POA 2019} & \\
\hline & \multicolumn{2}{|r|}{ Closing Remarks } & Kazuomi Kario (Japan) \\
\hline $14: 00-15: 30$ & \multicolumn{2}{|r|}{ Hope Asia Network } & \\
\hline \multicolumn{4}{|c|}{$\begin{array}{l}\text { Poster Session } \\
\text { Exhibition Hall 2-CD, 1F, Miyakomesse }\end{array}$} \\
\hline \multirow[t]{5}{*}{$10: 00-11: 00$} & \multicolumn{2}{|c|}{ Poster Session II } & \\
\hline & \multicolumn{2}{|c|}{ P3 Measurement \& Assessment of Vessels/Blood Pressure } & $\begin{array}{l}\text { Chair: } \\
\text { Julio A. Chirinos (USA) } \\
\text { Takafumi Okura (Japan) }\end{array}$ \\
\hline & MP-16 & $\begin{array}{l}\text { Comparison of Indices of Central and Peripheral Blood } \\
\text { Pressure for Prediction of Cardiovascular Events in Short } \\
\text { Term ( } 25 \text { Months) Follow-Up }\end{array}$ & Junli Zuo (China) \\
\hline & MP-17 & $\begin{array}{l}\text { Usefulness of Echocardiographic-Based Left Ventricular } \\
\text { Hypertrophy in New Onset Atrial Fibrillation }\end{array}$ & Jiyeon Hong (Korea) \\
\hline & MP-18 & $\begin{array}{l}\text { Renal Resistive Index is an Early Predictor of Future } \\
\text { Development of Anemia in Patients with Hypertension and } \\
\text { Chronic Kidney Disease }\end{array}$ & Muneyoshi Tanimura (Japan) \\
\hline
\end{tabular}


Abstracts: The Pulse of Asia 2018 Kyoto

\begin{tabular}{|c|c|c|}
\hline MP-19 & $\begin{array}{l}\text { Novel Measurement of Endothelial Function Assessed by } \\
\text { Short Time Flow-Mediated Vasodilation: Comparison with } \\
\text { Conventional Flow-Mediated Vasodilation }\end{array}$ & Shogo Matsui (Japan) \\
\hline MP-20 & $\begin{array}{l}\text { Cuff Based Oscillometric Measurements of the Augmentation } \\
\text { Index in Normotensive Japanese Individuals: Influence of Cuff } \\
\text { Suitability on the Augmentation Index }\end{array}$ & Masakazu Obayashi (Japan) \\
\hline MP-21 & $\begin{array}{l}\text { High Discordancy Between Unobserved Automated Office } \\
\text { Blood Pressure and Ambulatory Blood Pressure in a Tertiary } \\
\text { Hospital Cohort }\end{array}$ & Jiwon Seo (Korea) \\
\hline MP-22 & $\begin{array}{l}\text { The Predicting Value of WHtR to Atherosclerotic } \\
\text { Cardiovascular Disease }\end{array}$ & Liang Zheng (China) \\
\hline MP-23 & $\begin{array}{l}\text { Comparison Between 24-Hour Brachial and Central Blood } \\
\text { Pressures and Variability in their Associations with Target } \\
\text { Organ Damage in Untreated Chinese }\end{array}$ & Ying Wang (China) \\
\hline MP-24 & $\begin{array}{l}\text { A Case Report: Usefulness of Cibenzorin in Reducing } \\
\text { Obstruction at Left Ventricular Outflow Was Demonstrated by } \\
\text { Pulse Wave Analysis }\end{array}$ & Masafumi Satoh (Japan) \\
\hline P4 treat & nent, medication \& intervention & $\begin{array}{l}\text { Chair: } \\
\text { Der-Cherng Tarng (Taiwan) } \\
\text { Masahisa Shimpo (Japan) }\end{array}$ \\
\hline MP-25 & $\begin{array}{l}\text { Comparative Effects of Valsartan Plus Either Cilnidipine or } \\
\text { Hydrochlorothiazide on Nocturnal Home Blood Pressure in } \\
\text { Patients with Morning Hypertension }\end{array}$ & Takeshi Fujiwara (Japan) \\
\hline MP-26 & $\begin{array}{l}\text { Omega-3 Fatty Acid Improves Peripheral Artery Disease by } \\
\text { Lowering Remnant-Like Lipoprotein Cholesterol in } \\
\text { Hemodialysis Patients with Dyslipidemia: A Randomized } \\
\text { Controlled Trial }\end{array}$ & Yusuke Kobayashi (Japan) \\
\hline MP-27 & $\begin{array}{l}\text { Severity of Obstructive Sleep Apnea Is Not Associated with } \\
\text { Nocturnal BP Fluctuation in Elderly Hypertensive Patients } \\
\text { Receiving Amlodipine }\end{array}$ & Satoko Nozato (Japan) \\
\hline MP-28 & $\begin{array}{l}\text { Fimasartan, an Angiotensin II Receptor Blocker, Is Better } \\
\text { Effective in Reducing Pulse Pressure with Similar Safety in } \\
\text { Elderly Patients with Hypertension: K-MetS Study }\end{array}$ & Jeong Bae Park (Korea) \\
\hline MP-29 & $\begin{array}{l}\text { The Gender Difference of the Effect of Cocoa-Derived } \\
\text { Procynidin on Blood Pressure and Glucose Metabolism in } \\
\text { Pre-Diabetic Subjects }\end{array}$ & Kazuki Shiina (Japan) \\
\hline MP-30 & $\begin{array}{l}\text { Case Report: Increasing Ambulatory Blood Pressure } \\
\text { Variability and Pulse Pressure with Reverse Remodeling in } \\
\text { Heart Failure }\end{array}$ & Keisuke Narita (Japan) \\
\hline MP-31 & $\begin{array}{l}\text { Novel Hypertension Management Model of Care Resulting in } \\
\text { Decreased Blood Pressure in a West African Population }\end{array}$ & Isaac Kofi Owusu (Ghana) \\
\hline \multicolumn{3}{|c|}{ Poster (non-chaired) } \\
\hline $\mathrm{P}-04$ & $\begin{array}{l}\text { Ethnic Difference in Carotid-Femoral Pulse Wave Velocity and } \\
\text { Its Risk Profiles - Results from Two Community-Based Elderly } \\
\text { Cohort Studies }\end{array}$ & Qian-Hui Guo (China) \\
\hline P-05 & $\begin{array}{l}\text { Systolic Blood Pressure Difference Between Arms and Chronic } \\
\text { Kidney Disease in the Community Based } 10 \text {-Year Cohort Study }\end{array}$ & JongKwon Seo (Korea) \\
\hline P-06 & $\begin{array}{l}\text { Higher Cardioankle Vascular Index in 2nd Trimester of } \\
\text { Pregnancy Predicts Maternal Complications }\end{array}$ & Jeong Bae Park (Korea) \\
\hline P-07 & $\begin{array}{l}\text { The Relationship Between Nocturnal Dipping Pattern and } \\
\text { Morning Blood Pressure Surge in Hypertensive Patients }\end{array}$ & Bae Keun Kim (Korea) \\
\hline P-08 & $\begin{array}{l}\text { Supplementation of Vitamin D in Hypertensive Patients with } \\
\text { CKD Stage } 3 \text { and Vitamin D-Deficiency Does Not Improve } \\
\text { Arterial Stiffness }\end{array}$ & Enrique Rodilla (Spain) \\
\hline
\end{tabular}




\section{Abstracts}

SL1

Aging Kidney and Inflammation

Makoto Kuro-o

Division of Anti-aging Medicine, Center for Molecular Medicine, Jichi Medical University, Japan

Age-related histological changes in the kidney are associated with renal tubular damage and interstitial fibrosis, which are presumed as a result of non-infectious chronic inflammation. However, "pathogens" that induce such inflammation remain elusive. Here we propose that phosphate can be a pathogen that causes aging kidney.

Phosphate homeostasis is maintained primarily by the FGF23-Klotho endocrine axis. In response to phosphate intake, FGF23 (fibroblast growth factor-23) is secreted from the bone. The receptor for FGF23 is a heterodimer of FGF receptor (FGFR) and Klotho protein expressed in the kidney. FGF23 binding to FGFR-Klotho complexes induces suppression of phosphate resorption in proximal tubular cells and increase in phosphate excretion per nephron, thereby promoting urinary phosphate excretion. Although the FGF23-Klotho endocrine axis is indispensable for maintaining the phosphate balance, increase in FGF23 indicates increase in phosphate concentration in the proximal tubular fluid, which can trigger precipitation of calcium-phosphate crystals. Like pathogens, calcium-phosphate crystals have the ability to induce innate immune responses and cellular damages. Thus, high FGF23 can be regarded as a risk for renal tubular damage and inflammation, potentially leading to histological changes characteristic to aging kidney. In fact, increase in FGF23 and phosphate excretion per nephron caused by dietary phosphate load are associated with renal tubular damage and interstitial fibrosis in mice.

In mice, those histological changes resembling aging kidney occur when phosphate excretion per nephron exceeds $\sim 1.0 \mu \mathrm{g} /$ day. In humans, phosphate excretion per nephron in healthy young adults on regular diet is $\sim 0.5 \mu \mathrm{g} /$ day. Because the nephron number decreases with age, phosphate excretion per nephron is supposed to exceed $1.0 \mu \mathrm{g} /$ day in the aged, which can be a cause of aging kidney. The mechanism by which increase in phosphate excretion per nephron induces tubular damage will be discussed.

\section{SL2 \\ Housing Insulation and Health Intervention Study in Japan \\ Toshiharu Ikaga \\ Faculty of Science and Technology, Keio University, Yokohama, Japan}

In Healthy Japan 21, "a reduction in average systolic blood pressure by $4 \mathrm{~mm} \mathrm{Hg}$ " prevents an estimated 15,000 deaths from cardiovascular disease over a year. So, prevention of hypertension is prime task for Japan. Therefore, the purpose of this research is to clarify the relation between indoor thermal environment and blood pressure. Field surveys on home blood pressure, ambulatory blood pressure and indoor temperature are conducted in summer and winter from 2014 to 2018. An epidemiological survey of approximately 1,800 houses and 
3,600 residents living in warm and cold climate prefectures in Japan is conducted. This study is measuring indoor temperature and humidity, and residents' blood pressure and physical activity in winter. Controlling for personal factors, systolic blood pressure increased by 7.3 $\mathrm{mm} \mathrm{Hg}$ per $10^{\circ} \mathrm{C}$ decrease in indoor air temperature. Physical activity was measured by using a physical activity monitor. Participants wore the activity monitors daily. The indoor temperature and humidity were measured with thermo-hygrometers installed in the living room, bedroom, lavatory and corridor of each participant's house. The analysis resulted that although after adjusting biological factors, socioeconomic status and living-style etc., the higher the minimum temperature of lavatory and corridor are, the more physical activity inhabitants perform.

JS1-01

What Is SHATS?

Kazuomi Kario

Division of Crardiovascular Medicine, Jichi Medical University School of Medicine, Japan

A new concept of the synergistic vicious cycle of blood pressure (BP) variability and vascular disease (Systemic Hemodynamic ATherothrombotic Syndrome; SHATS) has recently been proposed. Broadly speaking, there are 2 common types of arterial disease: atherosclerosis with advanced atherosclerotic plaque, and arteriosclerosis with advanced arterial stiffness of large arteries, which is linked to small artery remodeling. The former is predominantly promoted by metabolic risk factors (e.g. diabetes, dyslipidemia), whereas the latter is most closely linked to aging and hypertension. Previous definitions of SHATS have included both types of vascular disease. Here, we clarify the concept of SHATS (Systemic Hemodynamic ArTeriosclerotic Syndrome) in a narrower sense, by separating the wellknown atherothrombotic mechanism of cardiovascular disease from the increasingly important arteriosclerotic mechanism.

\author{
JS1-02 \\ Strain Vessel Theory: An Evolutional Point of View \\ Sadayoshi lto \\ Tohoku University, Japan
}

In modern societies, lifestyle-related diseases such as hypertension, diabetes, and obesity are major causes of cardiovascular disease (CVD) and renal failure. One of the features of lifestyle-related diseases is that an apparently minor insult to the kidney, as manifested by microalbuminuria, is associated with a heightened incidence of CVD. Why? We may be able to find an answer in the process of the evolution of life. It is plausible that the structures and functions of vital organs and molecules essential for survival in the natural environment have now become villains that jeopardize our health in a modern society. The representative examples may include the circulatory systems that deliver blood supply to the sites crucial for survival such as brain stem, the renin-angiotensin-aldosterone system (RAAS), and renal metabolism of such substances as glucose and amino acids. I will discuss the relationship between the kidney, heart, and brain in lifestyle-related diseases from an evolutionary point of view. 


\section{JS1-03 \\ Blood Pressure Variability and Vascular Disease \\ Ji-Guang Wang \\ Department of Hypertension, Ruijin Hospital, The Shanghai Institute of Hypertension, Shanghai Jiaotong University School of Medicine, Shanghai, China}

Blood pressure variability is a complicated issue. It usually refers to blood pressure changes over a relatively short period of time, such as beat-to-beat or reading-to-reading variability, nighttime dipping and morning surge. Seasonal, visit-to-visit and day-by-day blood pressure variability have quite different mechanisms and probably clinical relevance as well. Non-invasive beat-to-beat blood pressure variability is used for the measurement of baroreflex sensitivity. The most relevant measurements of blood pressure variability for cardiovascular prevention should be reading-to-reading variability, nighttime dipping and morning surge, all of which can be obtained by ambulatory blood pressure monitoring, and have been shown to be predictive of cardiovascular disease. An even more complicated issue is that while blood pressure variability is a risk factor of vascular disease, the latter can also increase blood pressure variability. Arterial stiffness has been shown to precede the development of hypertension. In fact, it may also increase blood pressure variability both before and after hypertension is established. We may have to measure and treat both blood pressure variability and arterial stiffness. Randomized controlled therapeutic trials are required in this regard.

\section{JS1-04 \\ Blood Pressure Variability and Organ Damage \\ Mitsuru Ohishi \\ Kagoshima University, Japan}

From 2010 reported from prof. Rothwell, BP variability is focused on BP control. They reported the visit-to-visit variability of BP is associated with the occurrence of stroke and cardiovascular diseases. After the Lancet reports, many reports about the relationship between visit-to-visit variability of BP and other organ damages. Our group reported visit-tovisit variability of BP influenced on the occurrence of stroke and cardiovascular events in patients with hypertension, and also renal function and renal arterial resistance. Other group suggested visit-to-visit variability influenced on white matter lesions in the brain and reduction of cognitive function with atrophy of the hippocampus. According to these evidences, visit-to-visit variability is related to vascular remodeling. Other many studies about day-byday variability using home BP is also related to the occurrence of cardiovascular events. In this session, I would like to the evidences about BP variability with vascular remodeling in mind. 


\author{
JS1-05 \\ Exercise $=$ Severe Hemodynamic Stress $=$ Increased Risk? \\ Bo Fernhall, Ph.D. \\ University of Illinois at Chicago, Chicago, IL, USA
}

Acute aerobic maximal exercise produces substantial (severe) hemodynamic stress. Heart rate increases 2-3 fold from rest to maximal exercise and stroke volume may double. This translates to an increase in cardiac output from $4-5 \mathrm{l} / \mathrm{min}$ to $15-25 \mathrm{~L} / \mathrm{min}$, or even higher, depending on the fitness level of the individual. The changes in central blood flow are also coupled with an increase in blood pressure, often to levels around 200/80-90 or higher. Resistance exercise produces less of an increase in cardiac output but a much higher increase in blood pressure. This leads to the question: "Do these high levels of hemodynamic stress cause an increase in CV risk?" Data on the acute risk of cardiac events during exercise, suggest that risk is increased 3-7 times or even higher, during high intensity exercise such as running or cycling compared to non-exercise times. Furthermore, recent data suggest that acute severe exercise, such as running a marathon running, transiently decreases cardiac function. Arterial stiffness may increase following aerobic exercise, and definitely increases acutely following resistance exercise. Furthermore, acute firefighting decreases both cardiac and arterial function even in young firefighters. These findings are coupled with changes in coagulation and fibrinolysis that may increase the risk of acute events in vulnerable individuals. Very well trained older athletes also exhibit signs of greater amounts of atherosclerotic plaque and calcification compered to sedentary peers. Despite these findings, the data are very clear that both exercise training and chronic physical activity serve a protective role against CV disease, and the vast majority of epidemiological studies show the protective effect of chronic exercise/physical activity to be approximately $20-40 \%$. This presentation will attempt to reconcile these disparate findings and provide an overview of the current knowledge.

\title{
JS1-06 \\ Hypertension is a System Disruption of Dynamic Blood Pressure Regulation Mediated by Brain
}

Takuya Kishi

Department of Advanced Risk Stratification for Cardiovascular Diseases, Center for Disruptive Cardiovascular Medicine, Kyushu University, Japan

Long-term survival of hypertension still remains to be wrong, and we do not still overcome hypertension despite of various pharmacological and device therapies. We should now focus on the root of the evil in hypertension. As Dr. Guyton indicated, hypertension is a disruption of normal blood pressure homeostasis associated with rapid regulation of sympathetic nerve activity (SNA) and long-term renal pressure-diuresis relationship. SNA is determined by brain referring the afferent inputs from baroreceptor, chemoreceptor, and vagal nerve. I have demonstrated that; 1) renal afferent nerve stimulation worsen and vagal afferent stimulation attenuate baroreflex central component (transfer of blood pressure-brain-SNA), 2) baroreflex buffers fluid-volume changes through equal capabilities of improving the pressurediuresis relationship by sympathoinhibition and reducing urine volume by depressor response, 3) baroreflex failure disrupts blood pressure stability against volume infusion in spontaneously hypertensive rat (SHR) to a greater extent than in normotensive rat,, 4) excess 
salt intake worsen the disruption of blood pressure stability caused by baroreflex failure in SHR, and 5) our novel artificial baroreflex system could normalize blood pressure homeostasis. Moreover, 6) angiotensin II type 1 receptor-induced oxidative stress with inflammation in rostral ventrolateral medulla, which is known as a vasomotor center, causes prominent hypertension with sympathoexcitation in SHR, and 7) Direct pharmacological blockade of central angiotensin II type 1 receptor or novel optogenetics against astrocytes improves blood pressure homeostasis in various hypertensive model rats. These results strongly suggest that brain works as a central processing unit integrating afferent inputs, and that hypertension is a system disruption of dynamic blood pressure homeostasis mediated by brain. In these aspects, we would like to propose the novel and future unmet brain-targeting autonomic modulation therapies for hypertension.

\section{SY1-01 \\ Basic Concepts and New Evidence of Vascular Biomarkers \\ Alberto Avolio \\ Department of Biomedical Sciences, Faculty of Medicine and Health Sciences, Macquarie University, Sydney, Australia}

Arterial blood pressure is accepted as a significant modifiable factor for reduction of cardiovascular risk. However, due to the inherent variability of blood pressure, there is continuing controversy on the thresholds of blood pressure in relation to diagnosis of levels of hypertension and subsequent treatment and management, resulting in modification of guidelines as data from population studies and clinical trials become progressively available. Data availability will become increasingly widespread due the rapid growth of wearable devices and access to cloud storage, providing large amounts of information on the variability of blood pressure in normal daily living. Although not yet established, blood pressure variability may become an important metric which will inform blood pressure measurement, analysis and treatment and management decisions. Identification of patterns of blood pressure variability with different time constants will contribute to advancement of the novel concept of Systemic Hemodynamic Atherothrombotic Syndrome (SHATS) where the effects of blood pressure variability interact with vascular function to affect end-organ damage.

This presentation will address underlying hemodynamic concepts involved in relationships of pulsatile pressure and flow and association of blood pressure variability and vascular stiffness. Pulse propagation phenomena will be addressed in the context of effect of pulsatile stress on the microcirculation and effects in organs of high flow. Vascular biomarkers will be assessed in terms of relationships of pulsatile pressure in the central aorta and peripheral arteries and pressure dependent and pressure independent indices. In relation to pulsatile stress on the vasculature affecting organ function, novel experimental evidence will be presented that suggests that cyclic mechanical stretch on cerebral vascular endothelial cells can potentiate expression of the amyloid precursor protein, thus establishing a possible link between large artery function, vascular stiffness, pulse pressure and blood pressure variability with potential vascular causes of cognitive impairment and dementia, leading to Alzheimer's disease. 


\section{SY1-02 \\ Novel Arterial Biomarkers}

Julio A. Chirinos, $M D, P h D$

University of Pennsylvania Perelman School of Medicine and Ghent University, USA

The clinical importance of pulsatile hemodynamics is increasingly recognized. Brachial blood pressure, the most widely hemodynamic metric used by clinicians, is only partially informative. Over the last several decades, multiple approaches for non-invasive hemodynamic assessment have been developed. Many of these approaches have undergone rigorous assessments regarding biophysical determinants, physiologic implications. However, more studies assessing their clinical utility remain to be done.

Multiple approaches to hemodynamics can be considered complementary and when interpreted appropriately, can provide a comprehensive characterization of the hemodynamic profile. "Deep" assessments of central pressures, pressure-flow relations and ventricular-arterial coupling is now possible with unprecedented clinical feasibility. Analytical techniques are varied and informative. Measurements of input impedance, wave reflection magnitude and timing, wave separation, lumped parameter models, wave intensity analysis and wave power analysis, can jointly provide an "arterial hemodynamic phenome". Novel analytical techniques can in turn facilitate the interpretation of the arterial phenome in individuals. These measurements may in turn facilitate the development of new therapeutic targets, or precision-medicine approaches with existing pharmacologic agents. Key objectives to move the field forward include: (1) More standardization of methods and analytic approaches; (2) Wider application of these techniques in clinical trials and human studies in general; (3) The identification of "clinically actionable" findings on arterial hemodynamic assessments, in which the arterial phenome aids in clinical decision-making, after this proves unequivocally useful in carefully designed clinical studies.

\section{SY1-03 \\ Central Blood Pressure and Pressure Wave Reflection in Early Stages of Cardiovascular Abnormalities}

Hirofumi Tomiyama

Department of Cardiology, Tokyo Medical University, Tokyo, Japan

The elevated central blood pressure and abnormal pressure wave reflection have been demonstrated risks for future cardiovascular events. Even so, their roles in the early stages of abnormalities in cardiovascular systems have not been fully clarified.

1. We determined whether any significant association exists between change in late systolic cardiac load with time, estimated by radial pressure waveform analysis, and development of cardiac hemodynamic stress in individuals with preserved cardiac function. In healthy Japanese men $(n=1,851)$. A stepwise multivariate linear regression analysis demonstrated that among the parameters of radial pressure waveform analysis and markers of arterial stiffness analyzed, only PP2 was significantly associated with serum NT-proBNP levels in study participants at both the first and second examinations ( 3 years' interval).

2. We conducted a mixed-model linear regression analysis of repeated-measures data to examine whether, in the pathogenesis of hypertension, wave reflection acts additively/synergistically with arterial stiffness to increase the blood pressure. The mixed model analysis demonstrated that SBP/DBP and baPWV have significant longitudinal mutual associations, 
and that the annual increases in SBP/DBP and baPWV were accelerated by each other. Nevertheless, rAI had a significant longitudinal association with baPWV (estimate $=2.487, p<0.01$ ).

Thus, abnormal pressure wave reflection may be associated with development of cardiac hemodynamic stress and also the development of hypertension via the augmentation of arterial stiffening.

\section{SY1-04}

Aortic Stiffness and Hemodynamics in Chronic Kidney Disease

Junichiro Hashimoto

Miyagi University of Education Medical Center, Tokyo, Japan

Noninvasive measurements of aortic stiffness, pressure and flow have been applied for clinical risk assessments in chronic kidney disease (CKD). Renal hemodynamics is under strong influence of aortic stiffness and hemodynamics. Recent studies suggest that aortic stiffening not only widens aortic pulse pressure and renal flow pulsatility but also increases diastolic reversal of intra-aortic flow contributing to renal flow. These pressure and flow changes can cause renal microvascular (glomerular) damage or ischemia, which is often seen in the elderly with stiffened aorta. In fact, significant associations have been demonstrated between aortic stiffness/hemodynamic abnormalities and albuminuria or glomerular filtration rate deterioration. It is also reported that 1 ) aortic stiffness and pulse pressure are generally elevated in CKD patients, 2) they can predict cardiovascular disease event risk, and 3) de-stiffening therapy with antihypertensive medication may improve long-term prognosis of end-stage CKD. These reports indicate clinical usefulness of aortic stiffness/hemodynamic monitoring in CKD patients. In this session, recent evidence will also be discussed.

\section{SY1-05 \\ FMD \\ Yukihito Higashi \\ Hiroshima University, Japan}

In 1986, endothelial function was measured for the first time in patients with atherosclerotic coronary arteries. Since then, several methods for assessment of endothelial function, such as endothelium-dependent vasodilation induced by intra-arterial infusion of vasoactive agents using coronary angiography, a Doppler flow guide wire, and a mercury-filled Silastic strain-gauge plethysmography, and flow-mediated vasodilation (FMD), reactive hyperemiaperipheral arterial tonometry, and vascular response using an oscillometric method have been performed in humans. Although vascular responses to intra-arterial infusion of vasoactive agents, such as agonists to stimulate nitric oxide (NO) release and antagonists of NO, should be considered as the gold standard for assessment of endothelial function, this technique is invasive and burdensome for the subjects. FMD elicited by shear stress-induced NO production from endothelial cells is now widely used worldwide. FMD has advantages and disadvantages in methodological aspects and clinical perspectives. 


\author{
SY1-06 \\ The Methodology, Clinical Use and Evidence of CAVI \\ Tomoyuki Kabutoya \\ Division of Cardiovascular Medicine, Jichi Medical University School of Medicine, Japan
}

The cardio-ankle vascular index (CAVI) is consistent with stiffness parameter $\beta$, is an index that evaluates artery stiffness theoretically not affected by blood pressure, and it is calculated using the heart-ankle pulse wave velocity (PWV) from the origin of the aortic valve to the ankle region with blood pressure measured at the upper arm.

CAVI is calculated automatically using the VaSera System (Fukuda Denshi, Tokyo, Japan). Measurement of CAVI requires placement of electrocardiogram electrodes on both wrists and a microphone for phonocardiography. There have been shown that the usefulness of CAVI as a predictor of cardiovascular events in studies including patients with atherosclerotic risk factors or a history of cardiovascular disease. However, population-based longitudinal studies have not yet reported for cardiovascular events.

In this session, I would like to overview the methodology, clinical use and evidence of CAVI.

\title{
SY1-07 \\ Recent Evidence on Brachial-Ankle PWV-Why It Is Better Than Initially Expected?
}

Masanori Munakata

Research Center for Lifestyle-Related Disease and Division of Hypertension, Japan

Organization of Occupational Health and Safety, Tohoku Rosai Hospital, Sendai, Japan

Pulse wave velocity is a most popular measure for arterial stiffness. Carotid-femoral PWV has been used as a gold-standard PWV measure. However, this measure has not been taken root in the general practice in Japan, possibly because of methodological difficulties. Instead, brachial-ankle PWV has been long used and the evidence has been accumulated. Measurement of this parameter is easy, and its reproducibility is good. The generality and validity of the methodology is guaranteed. Brachial-ankle PWV has been reported to consistently increase with most traditional cardiovascular risk factors. Prognostic significance has been reported not only in Japanese but also in Korean or Chinese population. We recently reported the results of an individual participant data meta-analysis including 14,673 Japanese participants. The results clearly established brachial-ankle PWV as an independent predictor of the risk of development of CVD in Japanese subjects. Thus the prognostic significance of the brachialankle PWV is far better than the images of the initial expectation. Why? Recent longitudinal study conducted in multiethnic middle-aged men has shown that greater progression of aortic calcification is associated with larger increase in brachia-ankle PWV even adjusted for multiple covariates. In young obese women, dynamic leg exercises on a vertical vibration platform significantly increased leg muscle strength and reduced carotid-femoral PWV, femoral-ankle PWV and brachial-ankle PWV compared with non-exercise controls. Moreover, the reduction in the brachial-ankle PWV was correlated with decreases in the femoral-ankle PWV, aortic systolic blood pressure, augmented pressure, and augmentation index. Interestingly decreases in the aortic systolic blood pressure was related to the decrease in brachial-ankle PWV more than carotid-femoral PWV. These data suggest that leg arterial stiffness could be involved in the regulation of central hemodynamics trough the control of reflex wave from the periphery. 
Abstracts: The Pulse of Asia 2018 Kyoto

\title{
SY1-08 \\ Ankle-Brachial Index (ABI) Is a Marker for Both Atherosclerosis and Arteriosclerosis
}

Akio Ishida

Department of Cardiovascular Medicine, Nephrology and Neurology, Graduate School of Medicine, University of the Ryukyus, Japan

In clinical practice, $\mathrm{ABI}$ is measured exclusively for the assessment of the existence of lower-extremity peripheral arterial disease (PAD) in patients with high risk of cardiovascular disease. A low ABI $(\leq 0.9)$ has been used for the diagnosis of PAD. An abnormally high ABI $(\geq 1.4)$, which is related to medial artery calcification, is often associated with advanced atherosclerosis in patient with diabetes and end-stage renal disease. Both a low ABI and an abnormally high $\mathrm{ABI}$ are indicators of systemic atherosclerosis and independent predictor of cardiovascular events and mortality. Normal ABI value is defined as 1.00-1.39. We reported that the ABI was lowest at $20 \mathrm{~s}$, increased with age until $60 \mathrm{~s}$, and decreased thereafter in screened Japanese cohort. ABI significantly increased over the mean observation period of 4.9 years in subjects aged $<50$ years, but not in subjects aged $\geq 50$ years. Compared with normal ABI (1.00-1.19), indices of arterial stiffness, including SBP, pulse pressure (PP) and baPWV, were higher in subjects with high normal ABI (1.20-1.39). A high normal ABI was associated with left ventricular hypertrophy assessed by ECG, prevalence of proteinuria and also asymptomatic cerebral microbleeds. Furthermore, we recently found that a high normal $\mathrm{ABI}$ was associated with renal small artery intimal thickening in patients with CKD. These results are compatible to our hypothesis that the increase in ABI with age occurs as a result of increasing arterial stiffness and/or wave reflection, and is thus associated with target organ damage, including heart, kidney and brain. By contrast, a low ABI or an abnormally high $\mathrm{ABI}$ in older adults or high-risk subjects represents advanced atherosclerotic PAD.

\author{
SY2-01 \\ Effects of Antihypertensive Drug Treatment on Blood Pressure Variability \\ Jeong Bae Park \\ JB Lab and Clinic, Seoul, Korea
}

Blood pressure (BP) is characterized by marked fluctuations over different time windows showing beat-to-beat variability, 24-hour variability, day-by-day variability, and visit-to-visit variability. Clinically, BPV is a well-known risk factor for cardiovascular events and is associated with target organ damage and all-cause mortality independent of office BP levels. Dayby-day BPV is independent of beat-to-beat BPV and may have much different effects on the prognosis of hypertension. Increased day-by-day home BPV is associated with cardiovascular risk, the severity of target organ damage in patients with hypertension or diabetes mellitus and cardiovascular mortality in a community-dwelling population. Reducing both BPV and mean BP has been recognized as a potential target for improved management of hypertension to prevent cardiovascular events, particularly stroke. Here will be discussed on the effects of antihypertensive drug treatment on BP variability in diverse conditions. 
Abstracts: The Pulse of Asia 2018 Kyoto

\author{
SY2-02 \\ Home Blood Pressure Variability Based on the Routine Self-Measurement \\ Kei Asayama \\ Department of Hygiene and Public Health, Teikyo University School of Medicine, Japan
}

Self-measurement of blood pressure at home has been widely recommended for the management of hypertension. Home blood pressure is a strong predictor of cardiovascular complications, and it allows for a greater number of readings. Such multiple home blood pressure readings can provide information on blood pressure variability over varying time periods, from day-to-day to seasonal changes, in the relatively controlled home environment. However, to identify blood pressure variability as a clinically meaningful cardiovascular risk factor is unexpectedly difficult because of the methodological issues that may affect the risk estimation, e.g., the application of variability indexes that are dependent on blood pressure level, and the limitation of end points to mortality. In this presentation, I will provide an overview of the current literature on home blood pressure variability and introduce our recent findings that support the benefit of long-term continual home blood pressure measurement.

\title{
SY2-03 \\ Prognostic Significance of Home BP Variability on Diabetic Nephropathy: KAMOGAWA-HBP Study
}

Emi Ushigome

Kyoto Prefectural University of Medicine, Japan

The purpose of long-term care for patients with diabetes is to prevent the development of diabetic complications. It is important to control blood pressure (BP) as well as blood glucose for the prevention of renal and cardiovascular events or death in patients with diabetes. In recent years, the usefulness of home BP (HBP) self-measurement has been documented. HBP has been shown to have better predictive power for target organ damage relative to that of clinic BP. Moreover, there has been accumulating evidence showing that HBP variability independent of HBP level has a prognostic significance for the progression of cardiovascular diseases. We have reported on a cross-sectional study of day-by-day HBP variability in correlation to macroalbuminuria in patients with type 2 diabetes from KAMOGAWA-HBP cohort (Hypertens Res. 2011). Moreover, we have examined the prognostic significance of HBP variability in the prospective 2-year study using the same cohort. This prospective study revealed that day-by-day HBP variability is an independent predictor of progression to macroalbuminuria (J Hypertens. in press).

On the other hand, the possibility of the drug-class differences on HBP variability has been shown. In our cohort study, HBP variability in the morning is lower in patients with type 2 diabetes treated with calcium channel blockers (CCB) than that in those treated with angiotensin II receptor blockers (ARB) and/or angiotensin angiotensin-converting enzyme inhibitors (ACE-I), and that treatment with CCB was significantly correlated with HBP variability independent of other potential cofactors (J Diabetes Investig. 2013). We have also shown that combination of ARB with CCB is superior to that with diuretics in reducing HBP variability in an open-label cross-over pilot study (J Am Soc Hypertens. 2017).

The presentation will describe and discuss the HBP control, especially focusing on dayby-day HBP variability, to prevent the development of diabetic complications in patients with type 2 diabetes. 


\section{SY3-02 \\ Prognostic Significance of Morning Hypertension Associated with Sustained Nocturnal Hypertension \\ Sung-Ha Park \\ Division of Cardiology Severance Cardiovascular Hospital, Yonsei University College of Medicine, Seoul, Korea}

Previous studies have shown that morning hypertension (defined as average of 4 measurements after awakening of more than 135/85 mm Hg by ABPM or morning home BP of more than $135 / 85 \mathrm{~mm} \mathrm{Hg}$ ) is associated with increased risk of cardiovascular events. However, it is unclear whether morning hypertension associated with sustained nocturnal hypertension and that associated with morning blood pressure surge differs in terms of their effects on cardiovascular target organ damage and clinical outcomes. In this talk, we will discuss in depth the effect of nocturnal hypertension on cardiovascular target organ damage and cardiovascular risk and discuss whether or not subtypes of morning hypertension differ in terms of their effect on organ damage and outcomes.

\section{SY3-03 \\ What Is the Contribution of Blood Pressure Measurement Accuracy in Systemic Hemodynamic Atherothrombotic Syndrome?}

James E. Sharman

Menzies Institute for Medical Research, College of Health and Medicine, University of Tasmania, Hobart, Australia

Systemic Hemodynamic Atherothrombotic Syndrome is the concept of residual risk from surges in blood pressure that cause cardiovascular events among people with apparently controlled (mean) blood pressure and cardiovascular risk factors. In essence, proposing that dynamic blood pressure responses will be more informative than mean blood pressure values for risk prediction and potentially for therapeutic intervention. Recent evidence suggests that measurement error in blood pressure under resting conditions could underlie the reason why dynamic blood pressure responses more effectively capture risk related to blood pressure. This evidence comes from individual participant level meta-analyses of cuff blood pressure compared with invasive blood pressure (at the aortic and brachial artery levels) among several thousand participants from the 1950s to the present day. These data show that there is a pressing need to improve blood pressure device accuracy and, in doing so, should help refine the identification of people with blood pressure abnormalities, including those with Systemic Hemodynamic Atherothrombotic Syndrome.

\section{SY4-01 \\ New Evidence and Perspectives in RDN \\ Raymond R. Townsend \\ University of Pennsylvania, Philadelphia, USA}

In this segment we plan to review new evidence on the efficacy and safety of RDN in patients with hypertension, specifically patients treated with medication, and patients not on 
antihypertensives. After the publication of the Symplicity HTN-3 results, significant concern about the efficacy of RDN in people with high blood pressure arose. We evaluated the existing data of all Symplicity studies at that time, and designed the ON-MEDS and OFF-MEDS RDN trials to determine whether RDN had a clear signal in untreated hypertension, and whether RDN had a clear signal in treated but not controlled hypertension. In the Summer of 2017 we showed that the OFF-MEDS trial of RDN had a clear ABPM signal of efficacy. In the Spring of 2018 we showed that RDN had a clear signal in ON-MEDS hypertension. In the time allotted, we will review the results of these two trials and thereby place RDN in the toolbox of technologies which may be useful in the future to address the issue of uncontrolled hypertension.

\section{SY4-02 \\ The Procedure of Renal Denervation for Resistant Hypertension \\ Yukako Ogoyama \\ Division of Cardiovascular Medicine, Jichi Medical University School of Medicine, Japan}

The procedure of renal denervation is basically similar to percutaneous renal angioplasty, and the device to be used is a guiding catheter (currently 6fr or more) and a guide wire used for usual renal angioplasty. Other generators for cauterization and devices to be inserted into the renal artery are needed.

Even after the SYMPLICITY HTN - 3 trial was announced, renal denervation was performed at the clinical site, and various types of devices are currently being developed. Now in Japan, available catheters are balloon type (energy at ultrasound) or spiral type (energy at radiowave).

The primary problem with this treatment technique is that no endpoint has been established to determine whether cauterization was definitely achieved. Recently, it was reported that the distribution of the sympathetic nerve is around the renal artery, and the distal side becomes closer to the renal artery lumen, suggesting that it is more effective to cauterize the distal side of the renal artery. Therefore, depending on the effective depth of the device, the procedure is changing in the direction of cauterizing more distal, while in the device with high depth of reach, it is regarded as 4 sites on one side by cauterization of only the trunk.

A clinical trial of a new device was just started in Japan last summer. In the future, it is considered that a procedure ensuring safety will be necessary while evaluating the effectiveness of RDN.

\section{SY4-03 \\ Perspectives in Renal Denervation in Asia \\ Kazuomi Kario \\ Division of Crardiovascular Medicine, Jichi Medical University School of Medicine, Japan}

The SPYRAL global trial clearly demonstrated renal denervation (RDN) contributes to reduce both clinic and 24-hr ambulatory blood pressure (BP) (Townsent, et al. Lancet 2017). This BP lowering effect of RDN was greater during sleep and early morning periods (Kario, et al. ACC2018). Especially the patients with the sympathetic hypertension such as obstructive sleep apnea (Kario,et al.Circ J. 2016;80:1404-1412; Kario,et al.J Clin Hypertens 2016) might be the responder of RDN, potentially who have benefit to reduce cardiovascular events (Kario,et al.Hypertension 2015; Kario.Prog Cardiovascu Dis 2016). After the HTN-Japan was stopped (Kario,et al. Circ J.2015), 3 clinical catheter-based RDN trials are now ongoing; the 
first, SPYRAL (Symplicity Spyral multielectrode catheter, Medtronic, CA); the second REQUIRE (ultrasound-based system, PARADISETM, ReCor Medical, Ronkonkoma, NY), the third IBERIS (TCD-16164 multielectrode catheter, Terumo Corporation, Tokyo) in Japan. Asians may be a responder of RDN (Kario,et al. Hypertensikon 2018). Considering ethnic differences would be important to achieve consensus on the positioning of RDN in Asian.

\section{SS-1-01 \\ Evidence and Perspective of ABPM in Japan}

Satoshi Hoshide

Division of Cardiovascular Medicine, Jichi Medical University School of Medicine, Japan

Ambulatory blood pressure (BP) monitoring has been accepted for diagnosis and treatment of hypertension. In addition, ambulatory BP monitoring can evaluate various BP variability. Jichi Medical School ambulatory blood pressure monitoring (JMS-ABPM) study initially demonstrated that increased morning BP surge was associated with stroke incidence in elderly hypertensive patients independently of 24 hour BP (Kario et al. Circulation 2003). Further research showed that morning BP surge was significantly higher in Japanese hypertensive patients than in Western hypertensive patients (Hoshide et al. Hypertension 2015). Therefore, the evaluation of morning BP surge using ABPM may be more important in Japanese than any other races.

Nondipping BP pattern was a risk of cardiovascular events in Japanese population without target organ damage assessed by carotid intima media thickness, while this association was not found in the population without (Kotruchin, Hoshide et al. J Clin Hypertens 2018). Increased short-term BP variability (weighted SD of 24 hour) was associated with cognitive dysfunction in elderly Japanese population with good BP control (Cho, Hoshide et al. Am J Hypertens 2018). Although home BP measurement has been widely used in Japan, ambulatory BP monitoring would provide clinical implication in selected population.

We have performed two nationwide prospective observational study using ABPM. First study is the Japan Ambulatory Blood Pressure Prospective (JAMP) study using conventional ABPM, which enrolled over 7,000 patients with one or more cardiovascular risk factors and followed. Changes in temperature and activity affect BP level. Second study is the HomeAcitivity ICT based JAMP (HI-JAMP) using new ABPM device equipped with measurement of temperature and activity. These studies would provide new evidence and clinical implication for the management of hypertension using ambulatory BP monitoring in Japanese population.

\section{SS-1-02}

\section{ABPM in China}

Ji-Guang Wang

Department of Hypertension, Ruijin Hospital, The Shanghai Institute of Hypertension, Shanghai Jiaotong University School of Medicine, Shanghai, China

$A B P M$ is reimbursed by China health insurance. The use of ABPM in China is increased substantially in the past decade, but remains extremely low. Hypertension is still diagnosed mainly by clinic blood pressure measurement, which is performed often in a non-standardized way. ABPM has been performed only in very few hypertensive patients. The use of ABPM in 
China apparently has to be improved. We are conducting a nationwide prospective registry of ABPM and home blood pressure monitoring in 61 hospitals. In the meantime, we are promoting the use of a web based system for the analysis and interpretation of ABPM. With the research project and the industry approach, it is hoped that the use of ABPM would be increasing to a high level in the next decade, and that the diagnosis of hypertension would be based on ABPM in most hypertensive patients.

\title{
SS-1-03 \\ Multicenter Ambulatory Blood Pressure Monitoring Registry in Korea \\ Jinho Shin \\ Division of cardiology, Department of Internal Medicine, College of Medicine, Hanyang University, Seoul, Korea
}

Multicenter Ambulatory Blood Pressure Monitoring (ABPM) Registry in Korea was launched in 2009 in 29 centers. Among them, 21 centers enrolled more than 50 cases and 7,546 subjects were enrolled. Various aspects of ABPM were studied and published. Discordance between clinic blood pressure and ambulatory blood pressure (ABP) such as whitecoat hypertension and masked hypertension according to the treatment status, the relationship between $\mathrm{ABP}$ and target organ damage such as left ventricular hypertrophy, and the relation of obesity to the diurnal blood pressure change were reported. Recently, blood pressure variability (BPV) and the related factors were analyzed. The BPV parameter such as standard deviation, coefficient of variance, and average real variability were reported in relation to white-coat effect. Relationship between physical activity and BPV was also analyzed. In the presentation, various aspects of ABPM in Korea will be presented and discussed.

\author{
LS1-01 \\ New Approaches for Evaluating and Managing Hypertension \\ Michael Weber \\ Professor of Medicine, Downstate College of Medicine, State University of New York, USA
}

Although hypertension by itself is a major risk factor for cardiovascular events, its risk increases even further in patients who have additional risk factors for these outcomes. The most common risk factors to be taken into account in evaluating patients with hypertension are diabetes, dyslipidemia, obesity and tobacco use. All these factors contribute to a lifelong transition that starts with early arterial disease, leads to target organ injury and ultimately major events and mortality. These factors account for almost $90 \%$ of myocardial infarctions worldwide. Renal abnormalities also are strongly associated with fatal and non-fatal cardiovascular events; most patients with advanced kidney disease in fact die from CV causes. There are many CV biomarkers that can be assayed in the blood, but clinical awareness of the major atherosclerotic factors can adequately guide practice. Major surrogate endpoints include left ventricular hypertrophy and measures of arterial stiffness, which become strong indicators of cardiovascular events. The new United States ACC/AHA Hypertension Guidelines, recognizing the importance of atherosclerotic risk, have now designated hypertension at the new lower BP of 130/80 mm Hg or higher, which means that a very large proportion of adults now have this diagnosis. For patients where high BP is the only risk factor, 140/90 mm Hg remains 
as the threshold for starting treatment. But for patients with increased concomitant risk, including those with diabetes or known CV disease, as well as all patients aged 65 or older, the threshold is now $130 / 80 \mathrm{~mm} \mathrm{Hg}$. There is strong evidence that treating patients to below $130 \mathrm{~mm} \mathrm{Hg}$ is associated with significant reduction in CV event rates. Interestingly, new Canadian and Australian guidelines are even more aggressive and recommend a target of $<120 \mathrm{~mm} \mathrm{Hg}$ in high risk patients. To aid in more accurate diagnoses for these important decisions, all guidelines now stress the use of ambulatory or home BP monitoring to help guide decision making.

\section{LS1-02 \\ The Steno-Stiffness Chart Deriving from Simultaneous Measurement of Ankle Brachial Pressure Index (ABI) and Brachial-Ankle Pulse Wave Velocity (baPWV) for the Wide Range Risk Stratification}

Akira Yamashina

Tokyo Medical University, Center for Health Surveillance and Preventive Medicine, Japan

Cardiovascular disease (CVD) remains major cause of morbidity and mortality in developed countries. However, it is not fully predicted by traditional risk factors. Thus, more specific and more accurate marker of subclinical organ damage is indispensable. Arterial stenosis and stiffness, two major aspects of vascular damages, are believed to be important risk markers of CVD.

$\mathrm{ABI}$ is known to be an indicator of arterial stenosis or occlusion. Subjects with ABI less than 0.9 are diagnosed as Peripheral Artery Disease (PAD) and subjects with ABI between 0.90 and 1.0 are classified PAD if their waveform of the ankle pulse is blunted, and their cardiovascular risk is very high.

Pulse Wave Velocity (PWV) is known to be an indicator of arterial stiffness. A metaanalysis of Japanese observational studies demonstrated the cut off value of brachial-ankle PWV (baPWV) of 1,800 cm/s for high and $1,400 \mathrm{~cm} / \mathrm{s}$ for moderate cardiovascular risk.

In this session, I introduce a new concept of steno-stiffness chart for risk stratification algorism deriving from simultaneous measurement of ABI and baPWV. First, a patient with an $\mathrm{ABI} \leq 0.9$ and patient with $\mathrm{ABI}$ between 0.9 and 1.0 with blunted pulse wave of ankle are considered at very high risk. Second, a patient with an $A B I>1.0$ and a baPWV $\geq 1,800 \mathrm{~cm} / \mathrm{s}$ is considered at high risk. Third, a patient with an $\mathrm{ABI}>1.0$ and baPWV between 1,800 and $1,400 \mathrm{~cm} / \mathrm{s}$ is considered at moderate risk. Finally, ABI $>1.0$ and baPWV $<1,400 \mathrm{~cm} / \mathrm{s}$ is considered at low risk. This stratification algorism is easily measured and would be applicable in the clinical or health screening setting.

\section{LS2-01 \\ The Clinical Evidence of CAVI and the Comparison of Reference Value in CAVI and baPWV in Coupling Registry}

Tomoyuki Kabutoya

Division of Cardiovascular Medicine, Jichi Medical University School of Medicine, Japan

Cardio-ankle vascular index (CAVI) is a measurement of arterial stiffness, and is widely used as indices of atherosclerosis. In Japan, brachial-ankle pulse wave velocity (baPWV) is generally used to measure PWV, and while the reference value for this parameter is specified 
in Japanese guidelines for the non-invasive vascular function test, the CAVI reference value has not been standardized.

We have been conducting Cardiovascular prognostic coupling study in Japan (Coupling Registry). We already measured CAVI and baPWV on the same day as a part of this ongoing nationwide registry. The association between CAVI and baPWV was positive and significant. We evaluated the CAVI reference value corresponding to baPWV 14 and $18 \mathrm{~m} / \mathrm{s}$ derived from the regression line.

The roles of CAVI are not only surrogate marker as vascular dysfunction, but also predictor of target organ damage and cardiovascular events. The usefulness of CAVI as a predictor of target organ damage and cardiovascular events has been shown in various studies. Based on past reports, we will propose criteria of CAVI for vascular failure. The criteria of CAVI was concordant with our findings in Coupling Registry. However, none of population-based longitudinal study has been reported the cutoff vale of CAVI for cardiovascular events. In now, it should be cautious to apply these cutoff values of CAVI to general population.

The results of this ongoing prospective study are expected to confirm the association between the CAVI reference value and cardiovascular events.

\section{LS2-02 \\ Evaluating the Benefits of Using CAVI as a Predictor of Cardiovascular Events in High-risk Patients in Japan (CAVI-J) \\ Toru Miyoshi \\ Department of Cardiovascular Medicine, Okayama University Hospital, Okayama, Japan}

The cardio-ankle vascular index (CAVI) was developed in Japan and is a blood pressureindependent index of arterial stiffness from the origin of the aorta to the ankle. In recent years, it has been studied by many researchers worldwide, and it is strongly anticipated that it will play a role as a predictive factor for arteriosclerotic diseases. This prospective multicenter study to evaluate the usefulness of the CAVI to predict cardiovascular events in Japan (CAVI-J) is a cohort study with central registration. Participants $(n=3,000)$ will be scheduled to enroll and data will be collected for up to 5 years from entry of participants into the study. To be eligible to participate in the CAVI-J study, individuals have to be aged between 40 and 74 years and have at least one of the following risk factors for arteriosclerosis: (1) type 2 diabetes mellitus; (2) high-risk hypertension; (3) metabolic syndrome; (4) chronic kidney disease (stage 3), or (5) history of coronary artery disease or noncardiogenic cerebral infarction. The primary endpoints of this study are cardiovascular death, nonfatal myocardial infarction, and stroke. The cutoff for CAVI against the incidence of cardiovascular events will be determined.

\section{LS2-03}

Arterial Stiffness, CKD, CVD

Raymond R. Townsend

University of Pennsylvania, Philadelphia, USA

In this segment we will review the role of arterial stiffness as a predictor of prevalent CVD, and as a predictor CD progression using the Chronic Renal Insufficiency Cohort of the USA, an NIH/NIDDK funded effort in the USA. We will cover the basics of how arterial stiffness 
is measured in CKD, and the findings in the CRIC Study which link arterial stiffness to prevalent and incident CKD, as well as CKD progression in the CRIC study. Both cross-sectional associations and longitudinal outcomes will be presented at this time. We will also draw upon studies of arterial stiffness in other populations, like those of the Japanese workforce study, to establish the role of arterial stiffness as a factor in incident CKD in that population.

\section{PL01-03 \\ Expert Panel Consensus Recommendations for Home Blood Pressure Monitoring in Asia: The HOPE Asia Network}

Sung-Ha Park

Division of Cardiology Severance Cardiovascular Hospital, Yonsei University College of Medicine, Seoul, Korea

During the 2016 International society of Hypertension Meeting in Seoul, South Korea, a panel of experts from the Asia/Pacific region joined together to put forth a consensus recommendation for the use of home BP monitoring for treatment of hypertension in the Asia/ Pacific region. The evidence was based on review of evidence from meta-analysis, randomized controlled trials and observation cohort studies with the emphasis given to studies in the region. We put forth 9 recommendations that will hopefully lead to more widespread use of home BP monitoring in the Asia/Pacific region. In this talk, we will discuss the each of the recommendations and the clinical evidence that served as the basis for each of the recommendations.

\section{PL01-04 \\ Morning Hypertension}

\section{Ji-Guang Wang}

Department of Hypertension, Ruijin Hospital, The Shanghai Institute of Hypertension, Shanghai Jiaotong University School of Medicine, Shanghai, China

Morning blood pressure monitoring could be a clinically relevant concept in the therapeutic management of hypertension and in the prevention of cardiovascular complications by defining and treating morning hypertension. Because antihypertensive medication is often taken in the morning, uncontrolled morning blood pressure during the trough effect hours could be a hallmark of inadequate choice of antihypertensive regimen, such as, the use of short- or intermediate-acting drugs, under-dosing of drugs, or no-use or under-use of combination therapy. To improve the management of hypertension in general and morning hypertension in particular, long-acting antihypertensive drugs should be used in appropriate often full dosages and in proper combinations. The clinical usefulness of antihypertensive drugs with specific mechanisms against morning blood pressure or split- or timeddosing of long-acting drugs in controlling morning blood pressure remains under investigation. 
Abstracts: The Pulse of Asia 2018 Kyoto

\author{
PL-02 \\ Blood Pressure Variability and Morning BP Surge \\ Guru Prasad Sogunuru \\ Apollo Hospitals, India, Kathmandu University, Nepal
}

Hypertension is a major risk factor for cardiovascular and cerebrovascular disease. Blood pressure has a circadian rhythm, has high measurements during the day period and less during night period. Also, the occurrences of cardiovascular events are frequent in the morning. Blood pressure variability (BPV) is the average variation of BP throughout the day, quantitated as the standard deviation (SD) of ambulatory blood pressure (ABPM) recordings. Morning blood pressure surge (MBPS), is the difference between morning pressure (measured 2 hours after awakening) and the lowest night time blood pressure. Many studies demonstrated MBPS is associated with adverse cerebrovascular and cardiovascular events. Target organ damages like left ventricular hypertrophy, arterial stiffness, carotid atherosclerosis, renal albuminuria have been associated with MBPS. As per the synergistic resonance hypothesis proposed by Kario et al, MPBS would be potentiated by various components that are responsible for adverse events. The chief contributors being the autonomic nervous system and renin-angiotensin-aldosterone system (RAAS). The variabilities in blood pressure and related hemodynamic stress, in the background of the diseased micro and macrovasculature sets the vicious cycle, leading to end organ damage and the adverse events of systemic hemodynamic atherothrombotic syndrome (SHATS). There is a 2.7 fold increase in the risk of a future stroke in older patients with hypertension who were in the top decile of the morning BP surge. In Asians, compared to Whites, stroke (especially hemorrhagic stroke) is more common than myocardial infarction and there is steeper association between blood pressure and cardiovascular disease. Exaggerated blood pressure surges, morning or nocturnal are certainly contributory to end-organ damage and there is significant evidence supporting this. To study these dynamic features of BPV and MBPS in Asian population, we under HOPE-Asia net work will be discussing the available evidence.

\title{
PL-03 \\ Worldwide Cardiovascular Risk and Hypertension A Partnership of HOPE Asia and the World Hypertension League (WHL) \\ Michael A. Weber \\ Professor of Medicine, Downstate College of Medicine, State University of New York, USA
}

The creation of HOPE Asia provides a major new force in dealing with the high rate of cardiovascular events worldwide. It is also important to note that HOPE Asia has become an important partner of the World Hypertension League in its work in addressing the hypertension and cardiovascular disease epidemic, and in aiding research, education and clinical strategies in underserved parts of the world. All countries are experiencing the issues that come with the aging of populations, a problem exaggerated by increased consumption of high calorie and sodium foods, progressively more sedentary lifestyles and the avoidance of physical activity. Lipid disorders, diabetes and hypertension are all exaggerated by these factors. Although obesity is a major risk in predominantly White and Black populations, we should note that Asian communities, although not so commonly having the phenotype of obesity, are at high risk from even modestly elevated body weight. Very clearly, awareness 
and management of hypertension in low income countries is well below what we see in wealthier countries. A major focus for HOPE Asia and the WHL is improving blood pressure measurement reliability. Recent guidelines address the issues of white coat hypertension (high clinic BPs but normal BPs outside the clinic) and masked hypertension (normal clinic BPs but high BPs elsewhere). Obviously ambulatory and home BP measurements, when available and affordable, can be important in establishing correct diagnoses. It is also now well accepted that ambulatory and home BP measurements are even better than clinic BPs in predicting cardiovascular outcomes. Pivotal work in Japan has emphasized the importance of different times of day for CV risk, in particular the morning BP surge and also nighttime BP fluctuations that can be associated with poor sleep and sleep apnea. New affordable devices for home BP measurement appear to offer important progress in identifying and caring for hypertension and are also associated with improved treatment adherence by patients. This pioneering work in Japan is leading new approaches for hypertension management worldwide.

\section{PL-04 \\ The First Report from a Study to Define the Distribution of Type of Hypertension in Asia by Blood Pressure Monitoring at Home -Asia BP@Home- Kazuomi Kario \\ Division of Crardiovascular Medicine, Jichi Medical University School of Medicine, Tochigi, Japan}

Home blood pressure (BP) monitoring is endorsed in multiple guidelines as a valuable adjunct to clinic BP measurements for the diagnosis and management of hypertension. Several reports confirmed even when clinic BP is well controlled, uncontrolled masked morning hypertension with residual cardiovascular risk should still be a target for the management of hypertension. Furthermore, data from Japanese cohort studies have shown that there is a strong association between morning BP surge and cardiovascular events, suggesting that Asians in general may benefit from more effective control of morning BP.

We designed the Asia BP@Home study with the intention of collecting and analyzing data from across 12 territories in Asia, not only to determine relevant indices of BP variability in the home setting, but also to undertake an epidemiological evaluation of masked morning hypertension, white-coat hypertension, well-controlled and uncontrolled hypertension, using the same validated home $\mathrm{BP}(\mathrm{HBP})$ monitoring device and the same standardized method of HBP measurements.

This is the first report from the Asia BP@Home study. 


\section{YIA-01 \\ Impact of Indoor Temperature on Morning Home Blood Pressure in Winter: Baseline Data from a Non-Randomized Controlled Trial in Japan \\ Wataru Umishio ${ }^{1}$, Toshiharu Ikaga ${ }^{1}$, Kazuomi Kario ${ }^{2}$, Yoshihisa Fujino ${ }^{3}$, Tanji Hoshi ${ }^{4}$, Shintaro Ando ${ }^{5}$, Masaru Suzuki ${ }^{6}$, Shuzo Murakami ${ }^{7}$ \\ ${ }^{1}$ Keio University, Kanagawa, Japan, ${ }^{2}$ Jichi Medical University, Tochigi, Japan, ${ }^{3}$ University of Occupational and Environmental Health, Fukuoka, Japan, ${ }^{4}$ Tokyo Metropolitan University, Tokyo, Japan, ${ }^{5}$ University of Kitakyushu, Fukuoka, Japan, ${ }^{6}$ Tokyo Dental College, Chiba, Japan, ${ }^{7}$ Institute for Building Environment and Energy Conservation, Tokyo, Japan}

Objective: Mortality due to cardiovascular disease rises sharply in winter. Known as excess winter mortality, this phenomenon is partially explained by cold exposure-induced high blood pressure. We aimed to analyze the relationship between indoor temperature and blood pressure.

Design and Method: A survey was administered to households receiving subsidies for insulation retrofit as part of a non-randomized controlled trial. We collected data on the indoor environment and home blood pressure (HBP) of participants before and after insulation retrofit in winter. HBP was measured by participants twice daily, in the morning and evening, for two weeks. Data from the baseline survey in winter were obtained from 1,663 households and 3,001 participants. We analyzed the relationship between indoor thermal environment and HBP using a multilevel linear regression model with random intercepts, in which repeatedly measured day-level variables obtained from actual measurements (e.g., living room temperature, quality of sleep) were nested within individual-level variables obtained from the questionnaire survey (e.g., age, sex, body massindex, vegetable consumption, alcohol consumption, smoking status). The objective variable was systolic BP (SBP) in the morning $(n=50,155)$.

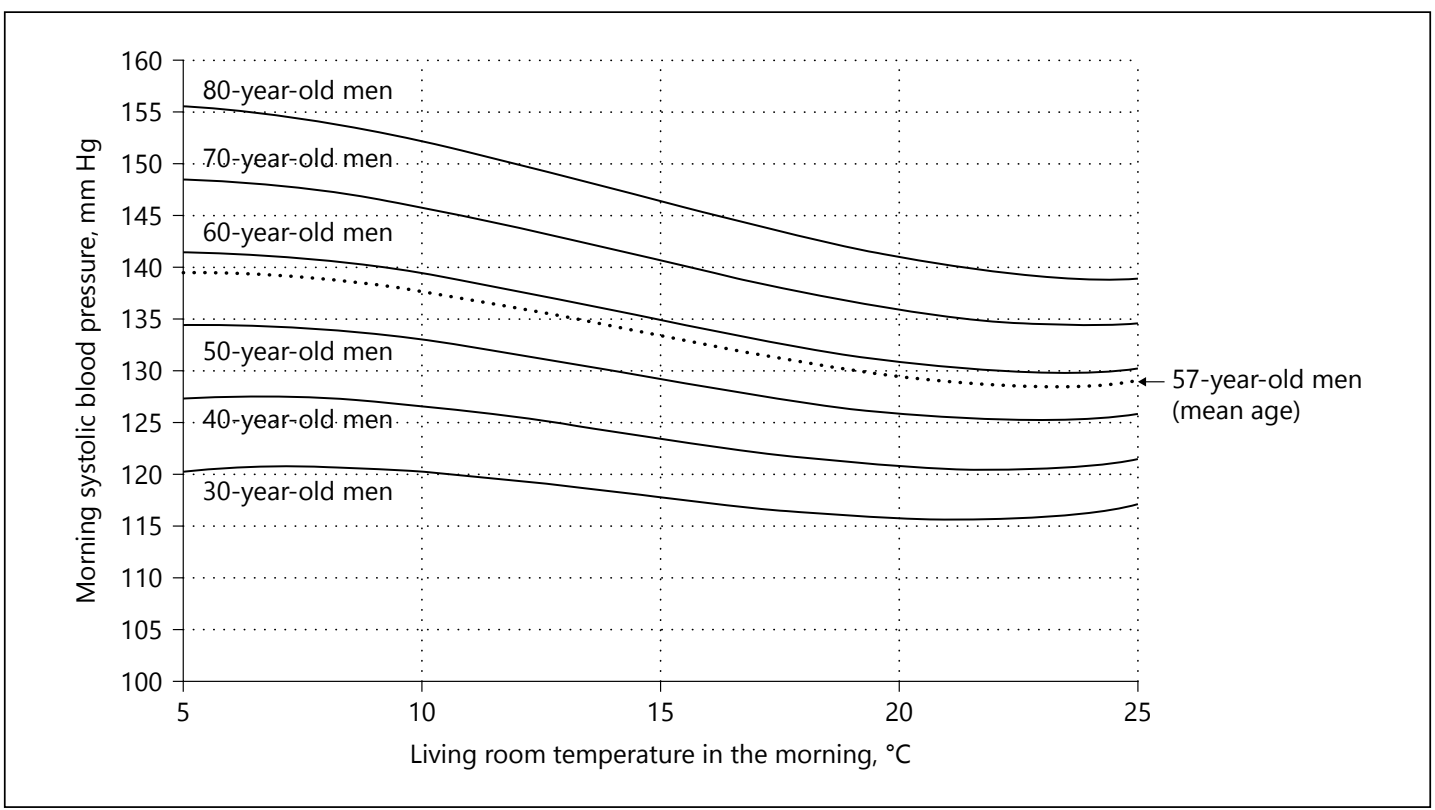

Fig. 1. The relationship between living room temperature and morning systolic blood pressure (in men). The average values for male participants were used. Vegetable consumption = regularly, exercise = rarely, current smoking status = non-smoker, alcohol consumption = every day, antihypertensive drug use = none (for Abstract no YIA-01). 
Results and Conclusion: There was a nonlinear relationship between morning SBP and living room temperature. The effect of room temperature on SBP was reduced at low and high room temperatures. Participants aged 57 years (mean age in this survey) had an average morning SBP increase of $6.5 \mathrm{~mm} \mathrm{Hg}$ per $10^{\circ} \mathrm{C}$ decrease in living room temperature. The temperature disparity between the living room and bedroom also affected SBP. There was a significant interaction between age and temperature, indicating a stronger effect of indoor temperature on SBP in older residents. These findings indicate that residents at high risk of cardiovascular events should maintain the indoor temperature within an appropriate range. They may also have important implications for managing hypertension and preventing cardiovascular events.

\section{YIA-02 \\ Association of Pulsatile Hemodynamics and Cognitive Dysfunction in Middle-Aged and Older Community Subjects: Results from The Longitudinal Aging Study of Taipei (LAST) \\ Chen-Hua Lin ${ }^{1}$, Hao-Min Cheng 1,3,4,5, Shih-Hsien Sung ${ }^{3,5}$ An-Chun Hwang ${ }^{2,8}$, Wei-Ju Lee ${ }^{2,9}$, Liang-Kung Chen ${ }^{2,8}$, Pie-Ning Wang ${ }^{9}$, Chen-Huan Chen ${ }^{1,3,5,7}$ \\ ${ }^{1}$ Institute of Public Health, ${ }^{2}$ Aging and Health Research Center and ${ }^{3}$ Faculty of Medicine, National Yang Ming University, Taipei, Taiwan; ${ }^{4}$ Center for Evidence-based Medicine, ${ }^{5}$ Department of Internal Medicine, ${ }^{6}$ Department of Neurology, ${ }^{7}$ Department of Medical Education, and ${ }^{8}$ Center for Geriatrics and Gerontology, Taipei Veterans General Hospital, Taipei, Taiwan; ${ }^{9}$ Department of Family Medicine, Taipei Veterans General Hospital Yuanshan Branch, Yuanshan Township, Yilan County, Taiwan}

Background/Objective: Increased arterial stiffness, wave reflection, and central blood pressure have been associated with worse cognitive performance. Excess pressure integral (XSPI), which derived from arterial reservoir-excess pressure analysis, is proposed as a novel index of circulatory dysfunction. The present study aimed to investigate whether XSPI may also associated with the cognitive functions.

Design and Method: In total, 692 community subjects (69\% female; mean age: $65.9 \pm$ 7.2; education $13.3 \pm 7.8$ years) from the Longitudinal Aging Study of Taipei (LAST) were enrolled. Participants completed echocardiography and applanation tonometry and XSPI was derived from the carotid pressure waveform. We used the Montreal Cognitive Assessment (MoCA) instrument Taiwan version to assess the global cognition and individual cognitive domains, including visual-spatial/executive function, naming, concentration, language, abstract, delayed recall and orientation. Cognitive impairment was defined by MoCA total score less than 26. Linear regression model were constructed to examine the relationship between pulsatile hemodynamic parameters and global cognition as well as individual cognitive domains.

Results: In univariate analysis, higher XSPI, carotid-femoral pulse wave velocity (cf-PWV), backward pressure amplitude (Pb), central systolic blood pressure (CSBP) and pulse pressure (CPP) were noted in 196 subjects with cognitive impairment. In linear regression analysis, only XSPI remained significantly associated with abnormal visual-spatial/executive function, after adjusting for other covariates, whereas none of pulsatile hemodynamic parameters significantly associated with the total MoCA score.

Conclusions: Our study demonstrated that subjects with impaired arterial reservoir function, increased arterial stiffness, more pronounced wave reflection and higher central blood pressure were associated with worse global cognitive performance. However, only XSPI were significantly associated with visual-spatial/executive function 
Abstracts: The Pulse of Asia 2018 Kyoto

independent of other covariates in multivariable adjustment. Excess pressure integral may be a novel biomarker in the relationship between vascular aging and cognitive dysfunction.

\section{YIA-03 \\ The Non-Dipper Blood Pressure Pattern Is an Independent Predictor of Cardiovascular Outcomes in Patients with Sleep-Disordered Breathing: The J-HOP Study \\ Hiroyuki Mizuno, Satoshi Hoshide, Kazuomi Kario \\ Division of Cardiovascular Medicine, Jichi Medical University School of Medicine, Japan}

Objective: In patient with sleep-disordered breathing (SDB), desaturations during sleep increase nighttime BP, which may result in increased cardiovascular (CV) events. However, the $\mathrm{CV}$ outcomes of SDB/non-dipper patients are unclear. The aim of the study is to investigate whether the non-dipper pattern is an independent predictor of CV outcomes in SDB patients.

Design and Method: We analyzed the 875 outpatients (mean age 62.7 years; $46 \%$ men; mean BMI 24.6) who have CV risk factors and have both ambulatory BP and pulse oximetry data. We classified the patients with a nocturnal systolic BP reduction $<10 \%$ as non-dippers, and the others as dippers. We defined the highest quartile of baseline $3 \%$ oxygen desaturation index (ODI) as the High ODI group (3\%ODI >10.1; $\mathrm{n}=214$ ) and the others as the Low ODI group (3\%ODI $\leq 10.1 ; \mathrm{n}=661$ ).

Results: During the mean 5.4-year follow-up, the incidence of CV events was significantly higher in the High ODI group compared to the Low ODI group $(6.5 \%$ [ $=14]$ vs. $3.5 \%$ [ $=$

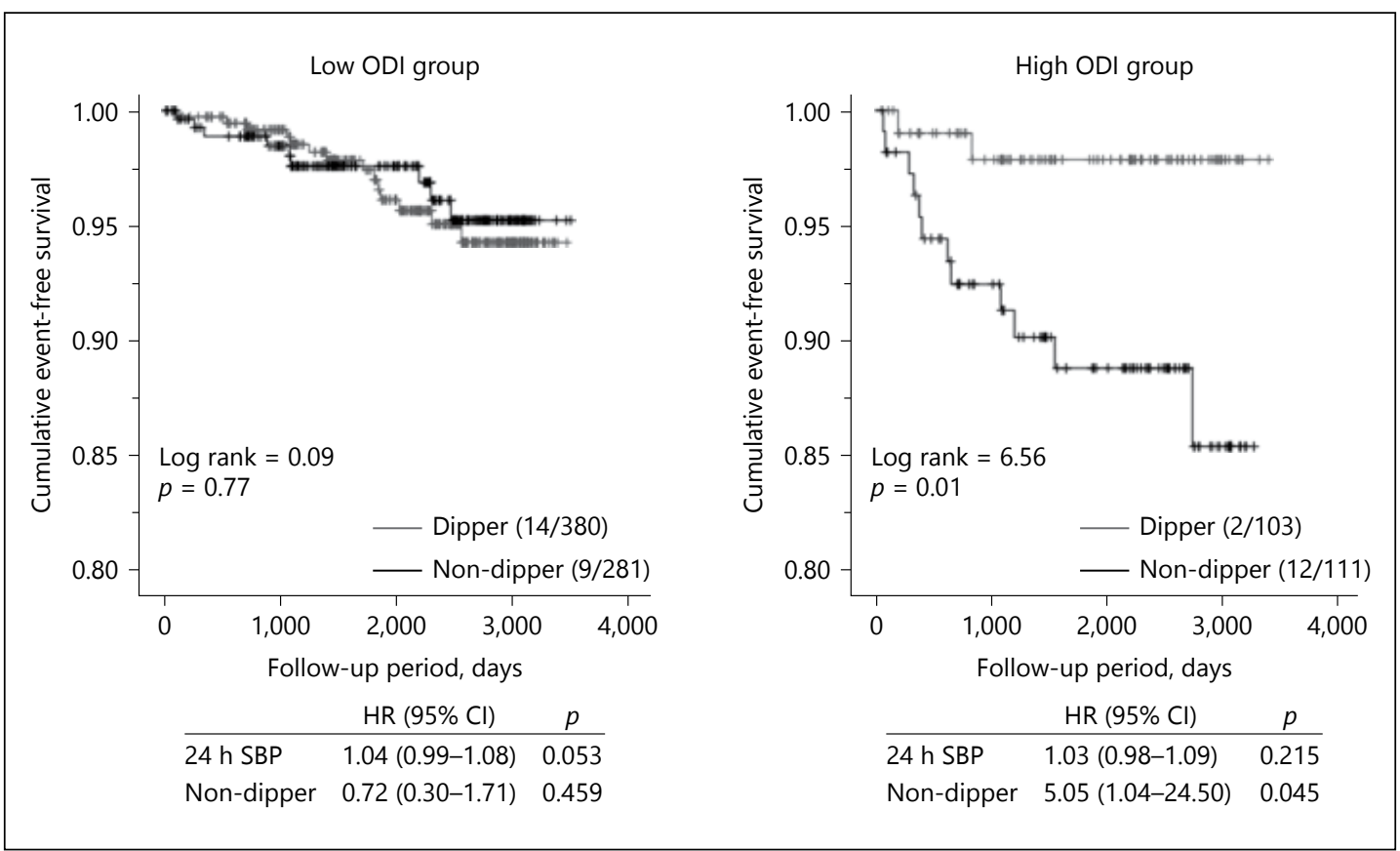

Fig. 1. Kaplan-Meier curve for cardiovascular events. Adjusted hazard ratios by age, sex, body mass index, smoking, alcohol, diabetes, hyperlipidemia, antihypertensive drugs, clinic systolic blood pressure (SBP) and 24-hr SBP (for Abstract no YIA-03). 
23], $\mathrm{p}=0.04)$. After adjustment for the other potential predictors, the non-dipper pattern was independently associated with increased CV events in the High ODI group (Hazard ratio 5.05, $95 \%$ confidence interval 1.04-24.50, $\mathrm{p}=0.04$ ) but not in the Low ODI group.

Conclusion: The non-dipper pattern was independently associated with increased CV events in the High ODI but not in the Low ODI group of Japanese with CV risk factors.

\author{
YIA-04 \\ The Association of Intracranial Arterial Stenosis with Home Blood Pressure \\ Level and Variability \\ Dongyan Zhang, Hui Chen, Qianhui Guo, Yibang Cheng, Qifang Huang, Changsheng Sheng, \\ Jiguang Wang, Yan Li \\ Shanghai Institute of Hypertension, China
}

Objective: Intracranial arterial stenosis (ICAS) is a major cause of ischemic stroke. However, the associations of ICAS with home blood pressure (BP) and variability remains unclear.

Design and Method: Outpatients not on antihypertensive medications were recruited from 2009 to 2013. ICAS was defined if the peak systolic flow velocities measured with transcranial Doppler sonography were respectively of at least $140 \mathrm{~cm} / \mathrm{s}, 120 \mathrm{~cm} / \mathrm{s}$, or $100 \mathrm{~cm} / \mathrm{s}$ at middle, anterior, or posterior and vertical cerebaral arteries. Home BP was self-measured by Omron HEM-7051 device for seven days. BP variability was assessed as variability independent of the mean, standard deviation, maximum-minimum difference, and average real variability.

Results: The prevalence of ICAS in the 801 participants (average age 51 years, 50\% males) was $7.9 \%$ (63 cases). Patients with ICAS compared to those without had significantly higher clinic (135.8 vs $131.9 \mathrm{~mm} \mathrm{Hg}, \mathrm{P}=0.01$ ) and home systolic BPs ( 134.8 vs $128.6 \mathrm{~mm} \mathrm{Hg}$, $\mathrm{P}<0.001)$. In multivariate-adjusted regression model, home systolic BPs, irrespective of at morning or evening, were associated with ICAS independently of other risk factors including any $\mathrm{BP}$ variability indices (OR, 1.47 to $1.82 ; \mathrm{P} \leq 0.005)$. However, after similar adjustment including home systolic BP, ICAS was only associated with seven-day morning systolic BP variability $(\mathrm{OR}, 1.35$ to 1.47 ; $\mathrm{P}<0.02)$, neither with evening $\mathrm{BP}$ variability $(\mathrm{P} \geq 0.47)$, nor any day-to-day BP variability indices ( $\mathrm{P} \geq 0.07$ ).

Conclusion: Asymptomatic ICAS was moderately prevalent in Chinese untreated patients. Both home morning and evening systolic BPs were important determinants of ICAS, and BP variability in the morning was also associated with ICAS.

\title{
0-01 \\ Hyperuricemia and Inflammation in the Increase in Arterial Stiffness and Development of Hypertension \\ Masatsune Fujii, Kazuki Shiina, Chisa Matsumoto, Shunsuke Komatsu, Kazutaka Kimura, Taishiro Chikamori, Akira Yamashina, Hirofumi Tomiyama \\ Department of Cardiology, Tokyo Medical University, Japan
}

Objective: Uric acid (UA) is a risk for the cardiovascular disease, but the underlying mechanisms of this development has not been fully clarified. The present prospective observational study examined whether UA is associated with the increase of arterial stiffness and the development of hypertension via inflammation. 
Method: In 3,274 middle-aged Japanese men (aged $42 \pm 9$ years old) without hypertension at the study baseline, the brachial-ankle pulse wave velocity (baPWV), blood pressure, serum C-reactive protein levels (CRP) and serum UA level were measured annually over a 9-year period.

Results: Hyperuricemia at the baseline was associated with a significant odds ratio for the presence of hypertension (odds ratio $=1.31 ; 95 \% \mathrm{CI}=1.16-1.49 ; \mathrm{p}<0.01$ ) and for the increase of the baPWV to the highest tertile range (odds ratio $=1.58 ; 95 \% \mathrm{CI}=1.02-2.46 ; \mathrm{p}=$ 0.01 ) at the end of the study period. The mixed model linear regression analysis revealed a significant and direct longitudinal association of hyperuricemia with each of increase of the baPWV (estimate $=6.59, \mathrm{p}=0.02$ ), the elevation of the blood pressure (estimate $=0.02, \mathrm{p}<$ 0.01 ) and elevation of the serum CRP level (estimate $=0.08 \times 10^{-1}, \mathrm{p}=0.02$ ). In addition, elevated serum CRP showed a significant direct longitudinal association with elevation of the blood pressure and increase of the baPWV (estimate $=91.30, \mathrm{p}<0.01$ ).

Conclusion: Hyperuricemia might also trigger affect inflammation, which can contribute, at least in part, to increased arterial stiffness and the development of hypertension. Thus, hyperuricemia may be a key player in the early stage of development of the cardiovascular disease.

\section{0-02 \\ Ascending to Descending Aortic Pulse Pressure Amplification in Children - Normal Aorta and Surgically Corrected Aorta \\ Tomoaki Murakami', Masahiro Shiraishi', Atsuhito Takeda ${ }^{2}$ \\ ${ }^{1}$ Department of Cardiology, Chiba Children's Hospital, ${ }^{2}$ Department of Pediatrics, Hokkaido University, Japan}

Objective: Pulse pressure amplification has been well known in adult, but not in children. Therefore, we examined it in patients with normal and surgically corrected aorta.

Design and Method: Fifty-four patients under 15 years old with a normal aorta were enrolled. The ascending and descending aortic pressure waveforms were recorded by a pressure sensor mounted catheter, and pulse pressure amplification, which was defined as the pulse pressure in the descending aorta minus that in the ascending aorta, was calculated. We also calculated the pulse pressure amplification in patients with aortic coarctation after surgical repair (15 patients) and transposition of the great arteries after arterial switch operation (12 patients), and compared the parameters with those in age-matched patients with normal aorta.

Results: In patients with normal aortic arch, the pulse pressure amplification was $4.5 \pm$ $2.7 \mathrm{~mm} \mathrm{Hg}$ and had a significant positive relationship with the patients' age $(r=0.36, \mathrm{p}=$ $0.009)$. The pulse pressure amplification was significantly attenuated in patients after aortic arch repair (aortic coarctation; $p<0.0001$, transposition of the great arteries; $p=0.0052$ ).

Conclusion: The degree of the pulse pressure amplification in children had a positive correlation with age, although it has a negative relationship with age in adults. The aortic surgery could attenuate the pulse pressure amplification. The patients after aortic surgery should be carefully observed, because it has been reported that the low pulse pressure amplification has a negative impact to the total mortality and the cardiovascular events. 
0-03

Which Is More Correlated with Hypertensive Organ Damage, Sleep Blood Pressure Assessed by Self-Measured at Home or Ambulatory Blood Pressure Monitoring?: The Japan Morning Surge-Home Blood Pressure (J-HOP) Study

Sirisawat Wanthong ${ }^{1,2}$, Tomoyuki Kabutoya ${ }^{1}$, Satoshi Hoshide ${ }^{1}$, Kazuomi Kario ${ }^{1}$

${ }^{1}$ Division of Cardiovascular Medicine, Jichi Medical University School of Medicine, Japan,

2Department of Medicine, Faculty of Medicine Siriraj Hospital, Mahidol University, Bangkok,

Thailand

Objective: To assess the associations with hypertensive target organ damage (TOD) of sleep SBP assessed by self-measured home blood pressure monitoring (HBPM) and ambulatory blood pressure monitoring (ABPM).

Method: Data of 1,008 participants in the J-HOP study who measured sleep BP using both HBPM, three times during sleep (2 AM, 3 AM and 4 AM) and ABPM during sleep were analyzed. Study participants were classified into 4 groups according to sleep SBP values: group 1, HBPM <120 mm Hg, ABPM <120 mm Hg; group 2, HBPM <120 mm Hg, ABPM >120

Fig. 1. $p$ value: unadjusted/adjusted age, gender/adjusted age, gender, office SBP (for Abstract no 0-03).

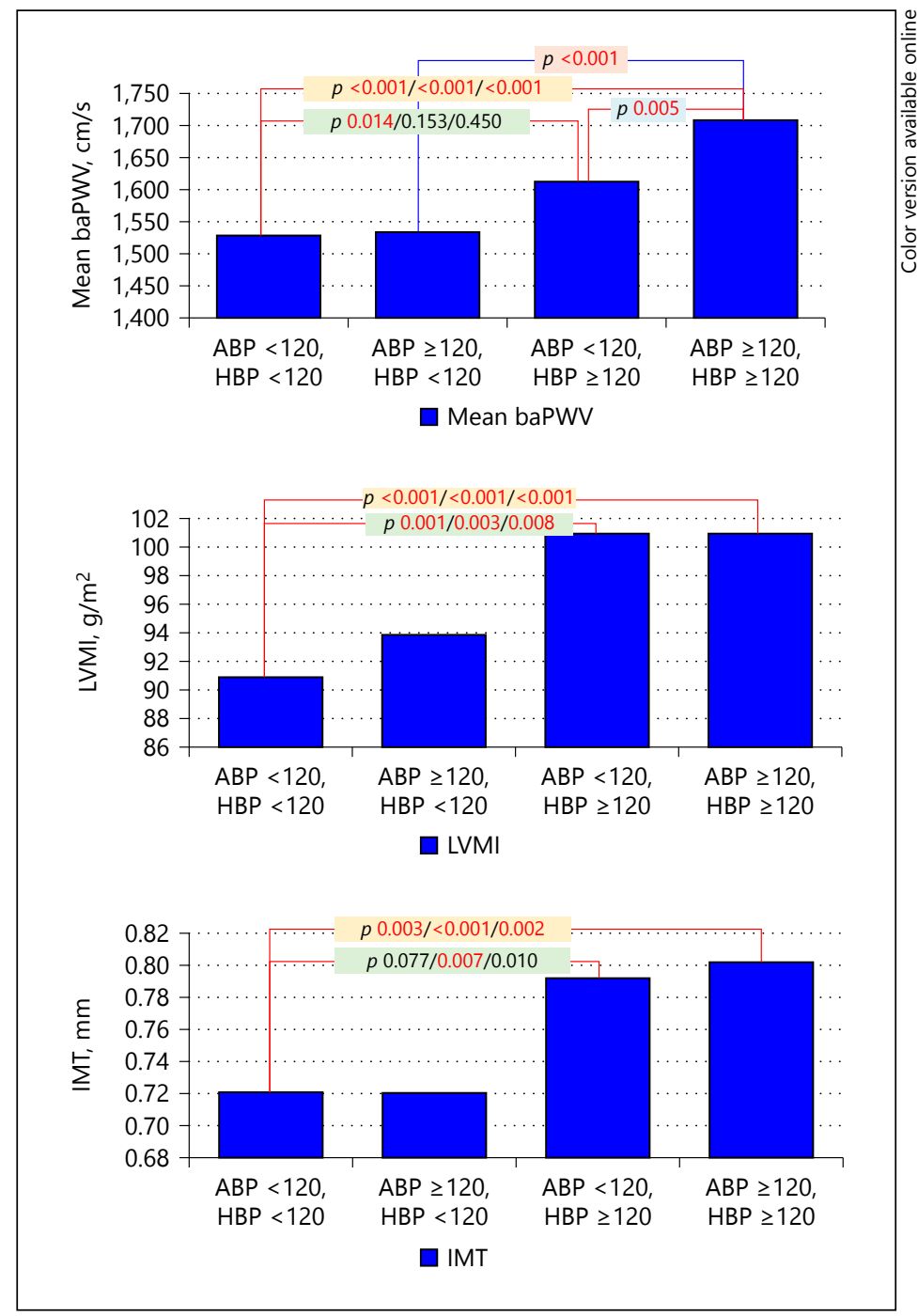


mm Hg; group 3, HBPM >120 mm Hg, ABPM <120 mm Hg and group 4, HBPM >120 mm Hg, ABPM $>120 \mathrm{~mm} \mathrm{Hg}$. Hypertensive TOD as indicated by brachial ankle pulse wave velocity (baPWV), left ventricular mass index (LVMI) and carotid intima media thickness (IMT), assessed in 946, 876 and 317 participants respectively, were compared among the 4 groups.

Results: Mean age was 63+11 years. The percentage of male participants was 49.9. Thirty-four, 10, 20 and 36 percent of the participants were classified into groups 1, 2, 3 and 4. For groups 1,2, 3 and 4, respectively, baPWV were 1,529 $\pm 293,1,536 \pm 265,1,616 \pm 255$ and $1,710 \pm 322 \mathrm{~cm} / \mathrm{sec}$, LVMI were $91 \pm 23,94 \pm 22,101 \pm 26$ and $101 \pm 28 \mathrm{~g} / \mathrm{m}^{2}$, and IMT were $0.73 \pm 0.14,0.72 \pm 0.18,0.79 \pm 0.15$ and $0.80 \pm 0.19 \mathrm{~mm}$. After age, gender and office SBP were adjusted, the baPWV, LVMI and IMT of groups 3 and 4 were significant higher than of group 1, In multivariate analyses, sleep SBP assessed by HBPM was an independent predictor of baPWV, LVMI and IMT, but that assessed by ABPM was an independent predictor of only baPWV.

Conclusion: Sleep SBP measured by HBPM was more closely associated with baPWV, LVMI and IMT than sleep SBP measured by ABPM.

\section{0-04 \\ Effects of a SGLT2 Inhibitor on Blood Pressure and Sympathetic Nervous Activity in Salt-Treated CKD rats}

Ningning Wan Akira Nishiyama

Department of Pharmacology, Kagawa University Medical School, Japan

Clinical studies have indicated that patients with chronic kidney disease (CKD) show saltdependent hypertension. In the present study, we investigated salt-sensitivity of blood pressure, its circadian rhythm and sympathetic nerve activity in experimental CKD rats. We also examined the effect of luseogliflozin, a selective inhibitor of sodium-glucose cotransporter 2 (SGLT2). Five-week-old male Wistar rats underwent uninephrectomy, and treated with adenine $(200 \mathrm{mg} / \mathrm{kg} /$ day) for 10 days. After 7 days stabilization with normal salt diet $(0.3 \% \mathrm{NaCl})$, high salt diet $(8 \% \mathrm{NaCl})$ was treated for 5 days. Thereafter, animals were again treated with normal salt diet $(0.3 \% \mathrm{NaCl})$. Blood pressure was continuously monitored by a telemetry system. We also analyzed a low frequency of systolic blood pressure, which reflects the sympathetic nerve activity. During treatment with normal salt diet, adenine-induced CKD rats showed normotensive non-dipper type of blood pressure. In these animals, high salt intake immediately increased mean arterial pressure (MAP). Five days after high salt diet intake, MAP was increased from 106 to $148 \mathrm{~mm}$ Hg. However, MAP was decreased to $96 \mathrm{~mm}$ $\mathrm{Hg}$ within 24 hours after switching to normal salt diet $(n=10)$. Treatment with a SGLT2 inhibitor (luseogliflozin: $10 \mathrm{mg} / \mathrm{kg} /$ day, p.o., $\mathrm{n}=10$ ) attenuated the high salt-induced elevation of MAP, which was associated with reduction in low frequency of systolic blood pressure. The present study demonstrates that CKD actually contributes to the development of saltdependent hypertension. Our data also suggest that treatment with a SGLT2 inhibitor attenuates the development of salt-induced hypertension by inhibiting sympathetic nerve activity in subjects with CKD. 


\title{
0-05 \\ Effect of Mean and Pulse Pressure on Fracture of Atherosclerotic Plaques Alberto Avolio', Alireza Rezvani-Sharif ${ }^{1,2}$, Mohammad Tafazzoli-Shadpour ${ }^{2}$ \\ ${ }^{1}$ Department of Biomedical Science, Faculty of Medicine and Health Sciences, Macquarie University, Sydney, Australia, ${ }^{2}$ Faculty of Biomedical Engineering, Amirkabir University of Technology, Tehran, Iran
}

Objective: Most myocardial infarctions occur as a result of atherosclerotic plaque rupture. Reliable evidence suggests that pulsatile blood pressure exposes atherosclerotic plaques to mechanical fatigue failure. In this study, the fatigue process and the subsequent crack propagation in realistic models of atherosclerotic plaques was investigated and the effect of mean and pulse pressure on the mechanism of plaque rupture was examined.

Design and Method: Eight atherosclerotic coronary arteries, obtained by endarterectomy surgery, were fixed in formalin and sliced by microtome. Sections were stained with Hematoxylin and Eosin and Elastica Van Gieson to highlight the plaque components including fibrous cap, lipid pool and calcium deposits. The geometry of atherosclerotic plaques was reconstructed in Abaqus software. After considering the material properties of plaque components and applying luminal pressure as an external load, the stress field within each model was estimated using finite element method. It was assumed that the initial crack occurred at the location of maximum stress concentration. The crack propagation was modeled in a stable way until it reached the lipid pool or the arterial wall. The number of fatigue cycles required for rupture of atherosclerotic plaques was calculated using fracture mechanic rules and the effect of pulse and mean pressure on fatigue life were examined.

Results: For the same pulse pressure of $40 \mathrm{~mm} \mathrm{Hg}$, increasing mean pressure from 93 to $133 \mathrm{~mm} \mathrm{Hg}$ resulted in reductions of fatigue life of approximately $25 \%$ in different cases. However, for the same mean pressure of $110 \mathrm{~mm} \mathrm{Hg}$, a decrease in the pulse pressure from 80 to $40 \mathrm{~mm} \mathrm{Hg}$ resulted in almost three-fold growth of fatigue life in different models.

Conclusion: Elevation of pulse pressure has greater influence than mean pressure on plaque rupture and can be considered as a risk factor for patients exposed to atherosclerosis disease.

\author{
MP-01 \\ Adhesion Molecules as a Novel Biomarker for Vascular Ageing \\ Sukrat Sinha \\ Centre of Biotechnology, University of Allahabad, Allahabad-211002, India
}

Adhesion molecules like CD2 play an important role in lymphocyte re circulation. In this study we took blood samples of males in the age group of 25-65 years from a local hospital in Allahabad. $1 \mathrm{ml}$ of blood was taken upon the ethical consent of the patient, family members and doctors. The lymphocytes were then separated by density gradient centrifugation. We focused on the expression of CD2 on CD4 and CD8 T cells of the subjects through FACS using fluorescent dyes. It was found with increase in the age there is a substantial decrease in the expression of CD2 which indicates this receptor may play an important role as a potential biomarker in vascular ageing. 
Abstracts: The Pulse of Asia 2018 Kyoto

MP-02

\section{A High Normal Ankle-Branchial Index Is Associated with Renal Small Artery Intimal Thickness in Chronic Kidney Disease}

Ryo Zamami, Akio Ishida, Tsuyoshi Miyagi, Masanobu Yamazato, Kentaro Kohagura, Yusuke Ohya

Department of Cardiovascular Medicine, Nephrology and Neurology, University of the Ryukyus School of Medicine, Japan

Objective: We have reported that ankle-branchial index (ABI) was positively correlated systolic pressure, pulse pressure and brachial-ankle pulse wave velocity (baPWV), indices of arterial stiffness, in a population of Japanese subjects with an $A B I \geq 1.0$. A high normal ABI $(1.20-1.39)$ was associated with proteinuria in younger subjects aged $<60$ years. The aim of this study was to investigate the relationship between ABI and renal small artery intimal thickness (SA-IT) in chronic kidney disease (CKD) patients.

Design and Method: We recruited 90 patients with CKD who underwent renal biopsy and ABI measurement by an automatic oscillometric method between 2010 and 2013. SA-IT severity was graded using semi-quantitative method. Patients aged $\geq 65$ years $(n=17)$ were excluded.

Results: The median age of patients was 42 years, with a range of 15-64 years (42\% women). Blood pressure was $122 / 74 \mathrm{~mm} \mathrm{Hg}$, and estimated glomerular filtration rate was $69 \mathrm{ml} / \mathrm{min} / 1.73 \mathrm{~m}^{2}$. Patients with high ABI ( $\geq 1.11$, median) had significantly higher age (47 vs. 38 years $\mathrm{P}<0.01)$, mean arterial pressure $(102 \mathrm{vs.} 92 \mathrm{~mm} \mathrm{Hg} \mathrm{P}<0.01)$, and baPWV $(1,446$ vs. $1,254 \mathrm{~cm} / \mathrm{s} \mathrm{P}<0.01)$ compared with patients with low ABI $(<1.11)$. Spearman's correlation analysis revealed a significant positive correlation between ABI and renal SA-IT grade $(\rho=0.39, \mathrm{P}<0.01)$. Multivariate logistic regression analysis showed that ABI was independently associated with high renal SA-IT grade (odds ratio per 0.1 increase in ABI was 1.11, $95 \%$ confidence interval $1.04-1.32, \mathrm{P}=0.01$ ).

Conclusion: A high normal ABI may reflect the existence of renal small artery sclerosis in patients with CKD.

MP-03

The Effect of Cardiac Valve Calcification on Long term Clinical Prognosis in Patients with Peripheral Vascular Disease Undergoing Percutaneous Transluminal Angioplasty

Ji Young Park' ${ }^{1}$, Seung-Woon Rha' ${ }^{2}$, Byoung Geol Choi', Ji Yeon Hong ${ }^{3}$

${ }^{1}$ Cardiology Department, Eulji General Hospital, Seoul, Korea, ${ }^{2}$ Cardiovascular Center, Korea

University Guro Hospital, Seoul, Korea, ${ }^{3}$ Cardiology Department, Hanil Hospital, Seoul, Korea

Background: Peripheral arterial disease (PAD) is known to be associated with poor outcomes, and cardiac valve calcification (CVC) is reported to be associated with future cardiovascular events.

Purpose: The aim of study is to evaluate the association of CVC with the long term clinical outcomes in patients (pts) with PAD who underwent percutaneous transluminal angioplasty (PTA).

Method: A total of 298 consecutive pts with symptomatic PAD who underwent PTA were enrolled for analysis. Study populations were divided into two groups; PAD with CVC $(\mathrm{n}=41)$ and PAD without CVC $(\mathrm{n}=257)$. CVC is defined as calcification and thickening of 
leaflets in aortic or mitral valve in the absence of obstruction of ventricular outflow. The incidence of restenosis, amputation rates and clinical outcomes were assessed at a follow-up of 2 years.

Results: Pts with CVC had higher incidence of wounds as the initial diagnosis for PAD (80.5\% vs. $56.0 \%, p=0.003)$, diabetes mellitus ( $92.7 \%$ vs.70.8\%, $\mathrm{p}=0.002)$, hypertension (87.8\% vs. $67.3 \%, \mathrm{p}=0.009)$, chronic kidney disease (43.9\% vs. $23.0 \%, \mathrm{p}=0.007$ ), need for dialysis (34.1\% vs. $16.0 \%, \mathrm{p}=0.009)$, and previous history of percutaneous coronary intervention $(26.8 \%$ vs. $13.6 \%, \mathrm{P}=0.037)$. At 8 months follow-up, patients with CVC had higher rate of total occlusion of the limb (83.3\% vs. 33.6\%, $p=0.023$ ). At 2-year follow up, the incidence of repeat PTA and major adverse cardiovascular events (MACE) was similar between the two groups, but the pts with CVC had higher amputation rate $(39.3 \%$ vs. $15.6 \%, p=0.005)$.

Conclusion: In this study, patients with CVC had more frequently presented with critical limb ischemia, higher rates of total occlusion and amputation rate at 2 years following successful PTA compared with those of PAD without CVC. More intensive therapies will be needed for this particular subset of risky patients.

\section{MP-04 \\ Association of Pulse Wave Velocity with Single Nucleotide Polymorphisms Related to Parathyroid Hormone \\ Yi-Bang Cheng ${ }^{1}$, Qian-Hui Guo ${ }^{1}$, Dong-Yan Zhang ${ }^{1}$, Ying Wang ${ }^{1}$, Qi-Fang Huang1, Chang-Sheng Sheng ${ }^{1}$, Ji-Guang Wang ${ }^{1}$, Jan A. Staessen ${ }^{2}$, Yan $L^{1}{ }^{1}$ \\ ${ }^{1}$ Shanghai Insititute of Hypertension, Ruijin Hospital, Shanghai Jiaotong University School of Medicine, ${ }^{2}$ Department of Cardiovascular Sciences, University of Leuven, Belgium}

Objective: Carotid-femoral pulse wave velocity (cfPWV) was associated with serum parathyroid hormone (PTH) in untreated Chinese. We investigated in the same cohort whether cfPWV, brachial-ankle (baPWV) and heart-brachial (hbPWV) pulse wave velocity (PWV) were associated with rs6127099 (CYP24A1) and rs4074995 (RGS14). A previously published genome-wide association study demonstrated that each additional copy of the T (rs6127099) or G (rs4074995) allele was associated with a 7\% or 3\% higher serum PTH, respectively.

Method: In 1,601 untreated Chinese patients (mean age, 51.0 years; $51.9 \%$ women), we measured cfPWV by tonometry (SphygmoCor) and baPWV and hbPWV by combined oscillometry and plethysmography (VP-2000 PWV/ABI analyser), serum PTH by an immunoassay, and genotypes by the SNapShot method.

Results: cfPWV, baPWV and hbPWV averaged 7.9, 14.6 and $5.5 \mathrm{~m} / \mathrm{s}$ and serum PTH 65.7 $\mathrm{pg} / \mathrm{mL}$. Genotype frequencies were in Hardy-Weinberg equilibrium, amounting to $41.7 \%$ (AA), 44.9\% (AT) and 13.4\% (TT) for rs6127099 and to 70.7\% (GG), 26.9\% (GA) and 2.3\% (AA) for rs4074995. With adjustments applied for sex, age, body mass index, heart rate and season, hbPWV was $0.05 \mathrm{~m} / \mathrm{s}(\mathrm{P}=0.042)$ lower with each additional copy of the minor allele (T) of rs6127099. In similarly adjusted analyses of 157 normotensive participants younger than 50 years, cfPWV was $0.32 \mathrm{~m} / \mathrm{s}(\mathrm{P}=0.004)$ higher per additional copy of the T allele. Sensitivity analyses additionally accounting for the total-to-HDL serum cholesterol ratio, plasma glucose, glomerular filtration rate and $24 \mathrm{~h}$ systolic blood pressure were consistent. No other association of PWV with the genetic variants reached significance.

Conclusion: With an increasing number of rs6127099 $\mathrm{T}$ alleles, arterial stiffness, as exemplified by PWV, was lower in all participants in a muscular artery (hbPWV), but higher in young normotensive participants in an elastic artery (cfPWV). 


\section{MP-05 \\ The Differential Impacts of Dialysis Vintage and Age on Hemodynamics and Autonomic Nerve Activity in Patients Under Hemodialysis}

Han-Kuei Wu ${ }^{1}{ }^{2}$, Hao-Min Cheng ${ }^{3}$, Ming-Yang Chang ${ }^{4}$, Hen-Hong Chang ${ }^{1,5}$

${ }^{1}$ School of Post-Baccalaureate Chinese Medicine, China Medical University, Taichung, Taiwan, ${ }^{2}$ Departments of Chinese Medicine, China Medical University Hospital Taipei Branch, Taipei, Taiwan, ${ }^{3}$ Department of Medical Education, Taipei Veterans General Hospital, Department of Medicine, National Yang-Ming University School of Medicine, Taipei, Taiwan, ${ }^{4}$ Kidney Research Center and Department of Nephrology, Chang Gung Memorial Hospital, Chang Gung University College of Medicine, Taoyuan, Taiwan, ${ }^{5}$ Department of Chinese Medicine, China Medical University Hospital, Taichung, Taiwan

Background: Cardiovascular event is one of the major morbidity and mortality in patients with end stage renal disease (ESRD). Both of age and dialysis vintage have impacts on cardiovascular function. The aim of this study is to investigate the relationships between heart rate variability (HRV) / pulse wave analysis (PWA) and age/dialysis vintage.

Method: We invited 70 patients with ESRD under regular hemodialysis. HRV and PWA were evaluated within one hour before hemodialysis. The relationships between HRV/PWA and age/dialysis vintage were analyzed by statistic methods.

Results: Baseline demographic characteristics showed negative correlation between dialysis vintage and dry weight, and negative correlation between age and phosphorus level. In HRV, there were positive correlation between dialysis vintage and InLF and LF\%, and negative correlation between age and LF\%. PWA data showed positive correlation between dialysis vintage and radial AI, and negative correlation between dialysis vintage and predialysis systolic blood pressure (SBP), central SBP, central MAP, and central pulse pressure. No correlation was noted between PWA and age.

Discussion: Higher AI represents a faster reflection wave and may implicate arterial stiffness, which is noted in the patients with longer dialysis vintage in our results. Furthermore, those patients had lower central blood pressure, which is possibly related to less cardiac output. Although, it is well known that aging is an important factor for higher AI and arterial stiffness, our results showed less significance in the association between age and AI. In the other way, LF\% as a factor of sympathetic activity had positive correlation with dialysis vintage but negative correlation with age. Higher sympathetic activity in patients with longer dialysis vintage may be consistent with the finding of higher AI.

Conclusion: Our study revealed that dialysis vintage may play a prominent role in sympathetic hyperactivity and arterial aging in patients with ESRD. 


\section{MP-06 \\ Lower Urinary Tract Symptoms in Men as an Early Phenotype of Endothelial Dysfunction}

Shogo Matsui ${ }^{1}$, Tatsuya Maruhashi ${ }^{1}$, Masato Kajikawa ${ }^{2}$, Shinji Kishimoto ${ }^{1}$, Haruki Hashimoto ${ }^{1}$, Takayuki Hidaka1, Ayumu Nakashima3 ${ }^{3}$ Kensuke Noma ${ }^{2}$, Yasuki Kihara ${ }^{1}$, Yukihito Higashi2,3

${ }^{1}$ Department of Cardiovascular Medicine, Hiroshima University Graduate School of Biomedical and Health Sciences, ${ }^{2}$ Division of Regeneration and Medicine, Medical Center for Translational and Clinical Research, Hiroshima University Hospital, ${ }^{3}$ Department of Cardiovascular Regeneration and Medicine, Research Institute for Radiation Biology and Medicine, Hiroshima University

Objective: To evaluate the relationships of vascular function and structure with lower urinary tract symptoms (LUTS) in men and women.

Method: This was a single center cross-sectional study. We investigated flow-mediated vasodilation (FMD) and nitroglycerine-induced vasodilation (NID) as vascular function, brachial-ankle pulse wave velocity (baPWV) as vascular structure, and LUTS assessed by International Prostate Symptom Score (IPSS) in 287 men and 147 women.

Results: IPSS was significantly correlated with conventional atherosclerotic factors, Framingham risk score, FMD, NID and baPWV. Moderate to severe LUTS was independently associated with the prevalence of cardiovascular disease (OR: 1.79, 95\% CI, 1.08-2.96; P = 0.02 ) after adjusting for traditional cardiovascular risk factors. In men, FMD and NID were lower in the moderate to severe LUTS group than in the none to mild LUTS group $(2.1 \pm$ $2.0 \%$ vs. $4.0 \pm 3.0 \%$ and $9.3 \pm 6.1 \%$ vs. $12.8 \pm 6.6 \%, \mathrm{P}<0.001$, respectively). baPWV was higher in the moderate to severe LUTS group than in the none to mild LUTS group (1722 \pm $386 \mathrm{~cm} / \mathrm{s}$ vs. $1509 \pm 309 \mathrm{~cm} / \mathrm{s}, \mathrm{P}<0.001$ ). After adjusting for confounding factors of LUTS, FMD was independently associated with decreasing IPSS $(\beta=-0.15, P=0.01)$, storage subscore $(\beta=-0.13, P=0.03)$ and voiding subscore $(\beta=-0.13, P=0.04)$ in men, but not in women. NID and baPWV were not independently associated with IPSS in either men or women.

Conclusion: In the present study, endothelial dysfunction was associated with LUTS in men. LUTS in men should be focused on as the early phenotype of atherosclerosis. Further studies are needed to determine whether LUTS can predict future cardiovascular events.

\section{MP-07 \\ Carotid Artery Atherosclerosis Is Closely Associated with Reduced Membrane Fluidity of Red Blood Cells in Normotensive and Hypertensive Subjects: An Electron Spin Resonance (ESR) Study}

Kazushi Tsuda

Cardiovascular Medicine, Kansai University of Health Sciences, Japan

It is well recognized that carotid artery atherosclerosis (CAA) may be involved in target organ damages in hypertension. On the other hand, it has been shown that abnormalities in physical properties of the cell membranes may strongly be linked to hypertension and other circulatory disorders. The present study was undertaken to investigate possible relationship between CAA and membrane fluidity (a reciprocal value of membrane microviscosity) in hypertensive subjects using an electron spin resonance (ESR)-method. The value of mean intima-media thickness (IMT) of the common carotid arteries determined by ultrasonograph was significantly greater in hypertensive subjects than in normotensive subjects (HT 1.140 \pm 
$0.063 \mathrm{~mm}, \mathrm{n}=30$, NT $0.947 \pm 0.039 \mathrm{~mm}, \mathrm{n}=34, \mathrm{P}<0.01)$. The order parameter $(\mathrm{S})$ for the spinlabel agent (5-nitroxide stearate) in red blood cells (RBCs) was significantly higher in hypertensive subjects $(0.730 \pm 0.002, \mathrm{n}=30)$ than in normotensive subjects $(0.715 \pm 0.002, \mathrm{n}=34$, $\mathrm{P}<0.001$ ), indicating that membrane fluidity was decreased in hypertension. The value of carotid IMT significantly correlated with the order parameters(S) of RBCs ( $r=0.441, n=64$, $\mathrm{P}<0.001)$. Furthermore, both carotid IMT and the order parameter (S) of RBCs correlated with plasma asymmetric dimethylarginine (ADMA:an endogenous nitric-oxide synthase inhibitor) levels, suggesting that CAA might be associated with impaired membrane microviscosity of RBCsand endothelial dysfunction. Multivariate regression analysisshowed that, after adjusting for confounding factors, carotid IMT might be an independent determinant of membrane fluidity of RBCs. The ESR study suggests that CAA might have a close correlation with impaired rheologic behavior of RBCs and microcirculatory dysfunction, at least in part, via an ADMAdependent mechanism.

\section{MP-08 \\ Different Impact of Nondipping Blood Pressure Pattern for Cardiovascular Outcome Between the Population with and Without Carotid Atherosclerosis: The Japan Morning Surge-Home Blood Pressure Study \\ Praew Kotruchin $^{1,2}$, Satoshi Hoshide ${ }^{1}, K_{\text {Kazuomi Kario }}{ }^{1}$ \\ ${ }^{1}$ Division of Cardiovascular Medicine, Jichi Medical University School of Medicine, Tochigi, Japan, ${ }^{2}$ Division of Emergency Medicine, Faculty of Medicine, Khon Kaen University, Khon Kaen, Thailand}

Risk of nondipping blood pressure (BP) in populations with different degree of carotid atherosclerosis on cardiovascular (CV) outcome is uncertain. We tested the hypothesis that differential impact of nondipping BP pattern would provide prognostic power for CV outcome in the populations with and without carotid atherosclerosis. From the Japan Morning SurgeHome Blood Pressure study, 493 patients (mean age 67.9 years, $47.5 \%$ male) with ambulatory BP monitoring (ABPM) and carotid intima media thickness (CIMT) data were analyzed. Nondipping BP pattern was independently associated with CV outcome in the population with CIMT $<1.1 \mathrm{~mm}$. This association was not found in the population with CIMT $\geq 1.1 \mathrm{~mm}$. Therefore, in hypertensive populations without CIMT at risk, physicians should consider ABPM to determine nocturnal BP patterns, which is alternative approach to assess CV outcome.

\section{MP-09 \\ Nocturnal Pulse Pressure Was Associated with Adverse Outcomes in Heart Failure with Preserved Ejection Fraction of Clinical Scenario 1 \\ Takahiro Komori, Satoshi Hoshide, Toshinobu Saito, Kazuomi Kario \\ Division of Cardiovascular Medicine, Jichi Medical University School of Medicine, Japan}

Background: The clinical scenario (CS) 1 heart failure (HF) had increased arterial stiffness and vascular failure, and frequently showed heart failure with preserved ejection fraction (HFpEF). Pulse pressure (PP) was associated with arterial stiffness and future cardiovascular events. We aimed to clarify that high PP is associated with adverse outcomes in CS1 HFpEF patients. 
Fig. 1. Kaplan-Meyer curves for main composite endpoints in the groups subdivided according to dipping BP patterns in subjects with (a) CIMT $<1.1 \mathrm{~mm}$ and (b) CIMT $1.1 \mathrm{~mm}$ (for Abstract no MP08).

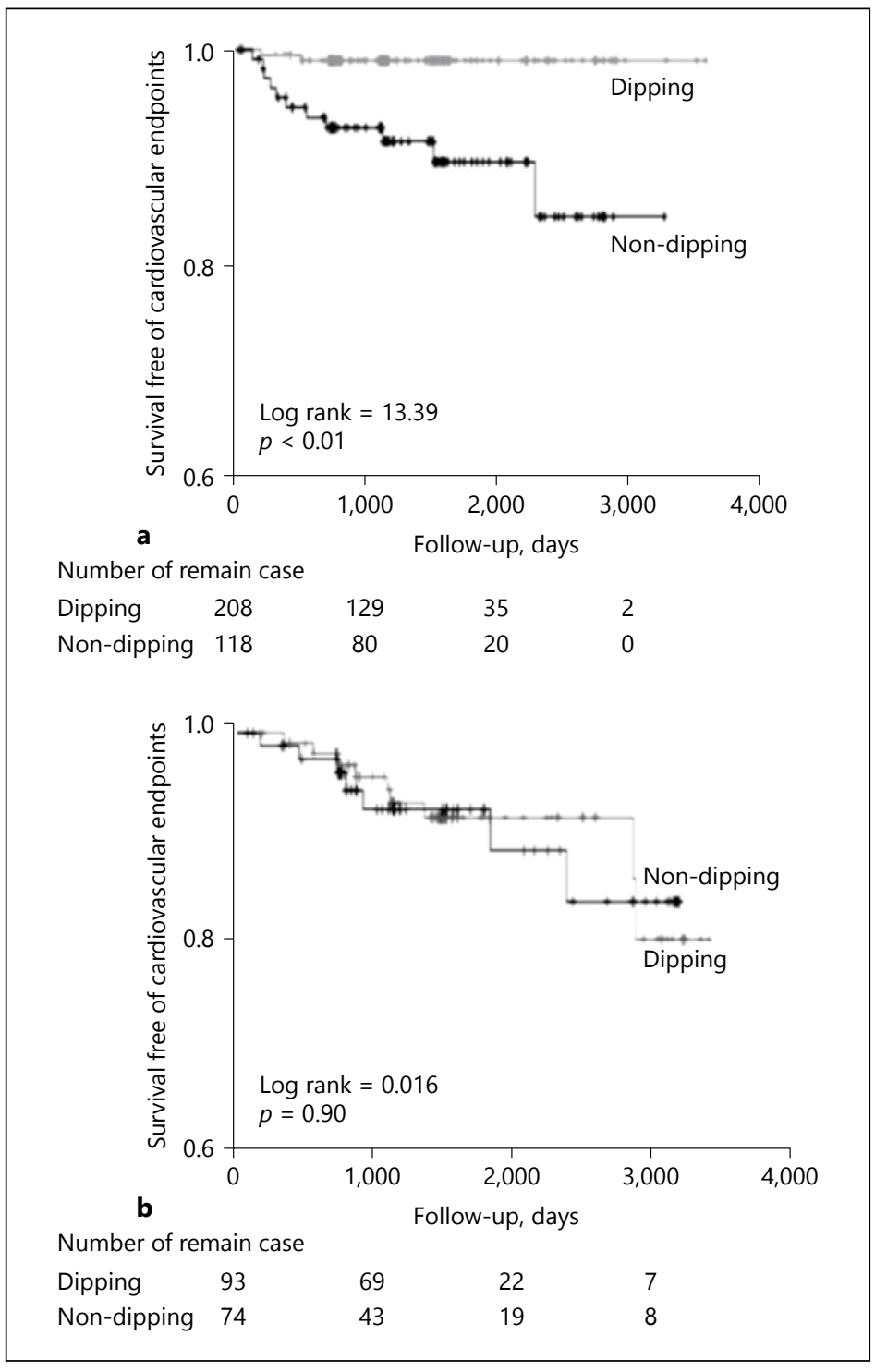

Methods: We enrolled 536 of hospitalized HF patients in our hospitals, and 285 of CS1 patients were analyzed (age, $71 \pm 12$ years; $60 \%$ male). Ambulatory blood pressure monitoring (ABPM) was performed and follow-up for $24 \pm 14$ months.

Result: Combined outcomes consists of all-cause mortality and cardiovascular events, and were seen in 122 cases. In HFpEF patients, nocturnal PP (per $10 \mathrm{~mm} \mathrm{Hg}$ increase) was significantly associated with combined outcomes (Hazard ratio 1.25, 95\% confidence interval [1.01-1.54], $\mathrm{p}=0.04$ ) adjusting for covariates, but was not in those with in HF with reduced ejection fraction.

Conclusions: Nocturnal PP was associated with outcomes in CS1 HFpEF patients. In CS1 HFpEF patients, PP was a marker of vascular failure and adverse outcomes. 


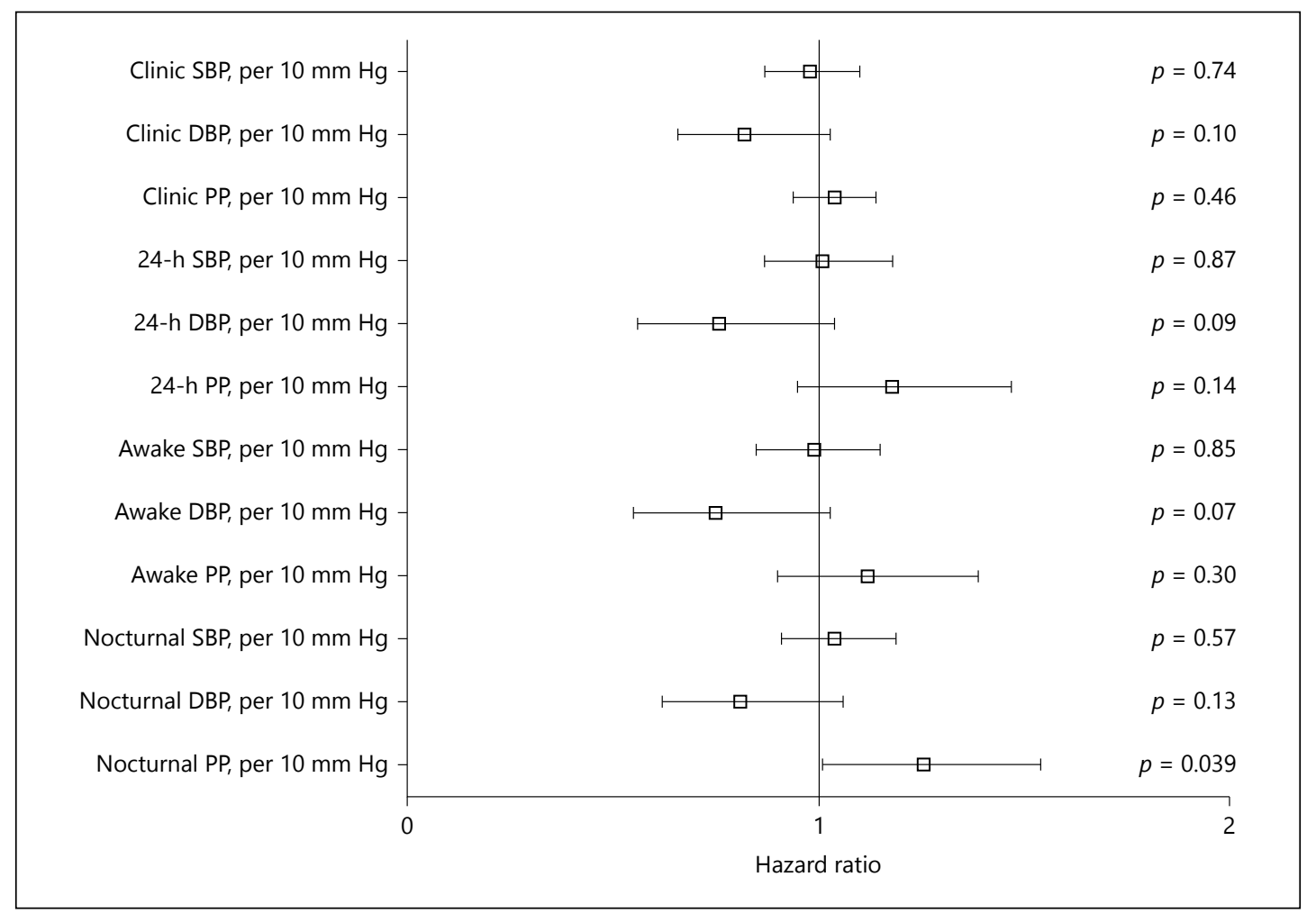

Fig. 1. Cox regression analysis for combined endpoints. SBP, systolic blood pressure; DBP, diastolic blood pressure; PP, pulse pressure. *Adjusted by hematocrit and creatinine, which were selected in the preliminary analysis with stepwise method included significant variables, such as age, sex, BMI, hematocrit, and creatinine (for Abstract no MP-09).

\title{
MP-10
}

Developing and Validating a New Precise Risk-Prediction Model for New-Onset Hypertension: The Jichi Genki Hypertension Prediction Model (JG Model)

\author{
Hiroshi Kanegae ${ }^{1}$, Takamitsu Oikawa ${ }^{1}$, Kenji Suzuki ${ }^{2}$, Yukie Okawara ${ }^{3}$, Kazuomi Kario $^{3}$
}

${ }^{1}$ Genkiplaza Medical Center For Health Care, ${ }^{2}$ The Japan Health Promotion Foundation, ${ }^{3}$ Jichi Medical University School of Medicine, Japan

Objective: No integrated risk assessment tools that include lifestyle factors and uric acid have been developed.

Design and Method: In accordance with the Industrial Safety and Health Law in Japan, a follow-up examination of 63,495 normotensive individuals (mean age 42.8 years) who underwent a health checkup in 2010 was conducted every year for 5 years. The primary endpoint was new-onset hypertension (systolic blood pressure/diastolic blood pressure $\geq 140 / 90 \mathrm{~mm} \mathrm{Hg}$ and/or the initiation of antihypertensive medications with self-reported hypertension).

Results and Conclusion: During the mean 3.4-years of follow-up, 7,402 subjects (11.7\%) developed hypertension. The prediction model included age, sex, body mass index, systolic blood pressure, diastolic blood pressure, lowdensity lipoprotein cholesterol, uric acid, proteinuria, current smoking, alcohol intake, eating rate, diastolic blood pressure by age, and body mass index by age at baseline and was created by using Cox proportional hazards models to calculate 
3-year absolute risks. The derivation analysis confirmed that the model performed well both with respect to discrimination and calibration $(n=63,495$; C-statistic $=0.885,95 \%$ confidence interval $=0.865-0.903 ; \chi^{2}$ statistic $=13.6$, degree of freedom $=7$ ). In the external validation analysis, moreover, the model performed well both in its discrimination and calibration characteristics $\left(\mathrm{n}=14,168 ; \mathrm{C}\right.$-statistic $=0.846 ; 95 \%$ confidence interval $=0.775-0.905 ; \chi^{2}$ statistic $=$ 8.7, degree of freedom = 7). Adding low-density lipoprotein cholesterol, uric acid, proteinuria, alcohol intake, eating rate, and body mass index by age to the base model yielded a significantly higher C-statistic, net reclassification improvement (NRI), and integrated discrimination improvement, especially NRInon-event $(\mathrm{NRI}=0.127,95 \%$ confidence interval $=0.100-0.152$; NRInon-event $=0.108,95 \%$ confidence interval $=0.102-0.117$ ). In conclusion, a highly precise model with good performance was developed for predicting incident hypertension using the new parameters of eating rate, uric acid, proteinuria, and body mass index by age.

\section{MP-11 \\ Prevalence and Characteristics of Exaggerated Morning Surge and Morning Hypertension in Chinese Patients: China Ambulatory and Home Blood Pressure Registry (ABPR)}

Qian-Hui Guo ${ }^{1}$, Yan Li ${ }^{1}$, Yuan-Yuan Kang ${ }^{1}$, Jian-Feng Huang ${ }^{1}$, Yu Dou ${ }^{2}$, Yan-Lun Su${ }^{3}$, Li-Juan Zhang ${ }^{4}$, Mei-Sheng Zheng ${ }^{5}$, Yi-Bang Cheng ${ }^{1}$, Ji-Guang Wang ${ }^{1}$

${ }^{1}$ Shanghai Institute of Hypertension, Ruijin Hospital, ${ }^{2}$ Department of Cardiology, Jiangsu Provincial Authorities Hospital, ${ }^{3}$ Department of Cardiology, Lianyungang Hospital of Chinese Medicine, ${ }^{4}$ Department of Electrocardiology, First Hospital of Xiamen City, ${ }^{5}$ Department of Hypertension, Wuhu Hospital of Chinese Medicine

Background: Exaggerated morning blood pressure surge (MBPS) and morning hypertension (MHT) was associated with worse prognosis. Little is known about the size of MBPS and the prevalence and determinants of MBPS and MHT in Chinese.

Design and Method: In the China Ambulatory and Home BP Registry (ABPR), we performed 24-h ambulatory BP monitoring. Exaggerated MBPS was a sleep-trough MBPS $\geq 35$ mm Hg. MHT was a mean ambulatory BP $\geq 135 / 85 \mathrm{~mm} \mathrm{Hg}$ in the first 2 hours after awakening.

Results: In the 3,575 registered patients (mean age, 56.7 years; women, 49.1\%; hypertension, 86.8\%), sleep-trough systolic MBPS averaged (SD) 20.5 (13.5) $\mathrm{mm} \mathrm{Hg}$, and 13.5\% had an exaggerated MBPS. MBPS was significantly associated with female sex, 24-h systolic $B P$, season and region distribution $(P \leq 0.03$ ). MBPS was higher in spring (effect size $\beta \pm S E$, $1.17 \pm 0.40 \mathrm{~mm} \mathrm{Hg}, \mathrm{P}=0.004)$ and winter $(1.75 \pm 0.38 \mathrm{~mm} \mathrm{Hg}, \mathrm{P}<0.001)$, and lower in summer $(-2.48 \pm 0.44 \mathrm{~mm} \mathrm{Hg}, \mathrm{P}<0.001)$ as compared to the average level of MBPS of whole population. MBPS also varied according to the regions of China, being higher in the Southeast $(1.63 \pm 0.49 \mathrm{~mm} \mathrm{Hg}, \mathrm{P}<0.001)$, and lower in the Northwest $(-2.64 \pm 0.70 \mathrm{~mm} \mathrm{Hg}, \mathrm{P}<0.001)$ as compared to the average level of the whole population. MHT was observed in $54.4 \%$ of all patients and $42.4 \%$ of treated hypertensive patients with office BP $<140 / 90 \mathrm{~mm} \mathrm{Hg} \mathrm{n}=$ 1225). MHT was significantly ( $\mathrm{P} \leq 0.01$ ) associated with male sex, age, body mass index, antihypertensive therapy, and region distribution $(\mathrm{P}<0.04)$. In contrast to the trend for MBPS, the prevalence of MHT was higher in the Northeast and lower in the Southeast, and the difference between the South and the North was significant $(\mathrm{P} \leq 0.03)$ in all seasons except autumn.

Conclusion: The size of MBPS in Chinese is modest, however, MHT is prevalent. MBPS and MHT had different determinants and differed across regions of China. 


\author{
MP-12 \\ Metabolic Syndrome Status Over 2 Years Predicts Incident Chronic Kidney \\ Disease: A 10-Year Prospective Cohort Study \\ Jang Young Kim, Ji Hye Hur, So Jin Lee \\ Department of Cardiology, Wonju College of Medicine, Yonsei University, Korea
}

Background: Single assessments have shown an association between metabolic syndrome (MetS) status and the development of chronic kidney disease (CKD), but the relationship between longitudinal changes in MetS status and the risk for CKD remains unclear. We investigated whether early changes in MetS status over two years modify the 10-year risk of CKD and proteinuria.

Method: A prospective cohort study was conducted in 7,251 subjects aged 40-70 years without CKD at baseline. The subjects were categorized into three groups according to their MetS status at two visits over two years: non-MetS (no MetS at either visit), intermittent MetS (positive for MetS at one assessment), and persistent MetS (positive for MetS at two assessments). The hazard ratio (HR) of new-onset CKD over a 10-year follow-up period was calculated using Cox models.

Results: During the 10-year follow-up period, 923 (12.7\%) developed CKD. Compared to the non-MetS group, the fully adjusted HR for new-onset CKD was the highest in the persistent MetS group (HR, 1.53; 95\% CI, 1.23-1.90), followed by the intermittent MetS group (HR, 1.29; 95\% CI, 1.04-1.59) ( $\mathrm{P}$ for trend <0.001). The HR for developing proteinuria was 1.79 (95\% CI, 1.15-2.79) in the persistent MetS group and 0.70 (95\% CI, 0.42-1.19) in the intermittent MetS group when the non-MetS group was considered as the reference group.

Conclusion: Changes in MetS status over the first two years influenced the 10-year risk of incident CKD and proteinuria. The risks of CKD and proteinuria increased when MetS was persistent during two visits over two years. Our findings suggest that monitoring and strictly controlling MetS are important in preventing renal function decline.

\author{
MP-13 \\ Gender Differences in Five Years Long Term Clinical Outcomes of Coronary \\ Artery Spasm in Korean Population Using Propensity Matching Analysis \\ Ji Young Park ${ }^{1}$, Seung Woon Rha' ${ }^{2}$ Byoung Geol Choi', Ji Yeon Hong ${ }^{3}$ \\ ${ }^{1}$ Departmet of Cardiology, Eulji Hospital, Eulji University, ${ }^{2}$ Cardiovascular Center, Korea \\ University Guro Hospital, Seoul, Korea, ${ }^{3}$ Cardiology Department, Hanil Hospital, Seoul, Korea
}

Background: Gender difference (GD) of cardiovascular disease have demonstrated in several studies.

Purpose: The aim of this study is to investigate GD in five years long term prognosis of coronary artery spasm (CAS) in Korean population.

Method: A total of 3,110 patients (1,575 men and 1,535 women) with CAS on intracoronary acetylcholine provocation test between 2004 and 2014 were enrolled for this study. Primary endpoint was major adverse cardiac events (MACE) including death, myocardial infarction, and revascularization, and secondary endpoint was recurrent angina requiring repeat coronary angiography.

Results: To adjust for confounders, we use the propensity score matching (PSM) analysis. Two matched groups (713 pairs, $n=1426$ ) were generated and baseline characteristics were balanced. The incidences of MACE and recurrent angina up to five years were similar between 
Abstracts: The Pulse of Asia 2018 Kyoto

Table 1. Clinical outcomes up to 5 years (for Abstract no MP-13)

\begin{tabular}{|c|c|c|c|c|c|c|}
\hline \multirow[t]{2}{*}{ Variables, N (\%) } & \multicolumn{3}{|c|}{ Entire Patients } & \multicolumn{3}{|c|}{ Matched Patients } \\
\hline & $\begin{array}{l}\text { Male } \\
(n=1,575)\end{array}$ & $\begin{array}{l}\text { Female } \\
(n=1,535)\end{array}$ & Log Rank & $\begin{array}{l}\text { Male } \\
(n=713)\end{array}$ & $\begin{array}{l}\text { Female } \\
(n=713)\end{array}$ & Log Rank \\
\hline MACE & $13(1.2)$ & $7(0.6)$ & 0.201 & $4(0.8)$ & $3(0.6)$ & 0.688 \\
\hline Total death & $5(0.4)$ & $3(0.3)$ & 0.502 & $1(0.1)$ & $2(0.4)$ & 0.581 \\
\hline Cardiac death & $2(0.1)$ & $1(0.0)$ & 0.578 & $0(0.0)$ & $1(0.1)$ & 0.322 \\
\hline Myocardial infarction & $4(0.2)$ & $2(0.1)$ & 0.432 & $1(0.2)$ & $0(0.0)$ & 0.316 \\
\hline Revascularization & $5(0.6)$ & $2(0.1)$ & 0.278 & $3(0.7)$ & $0(0.0)$ & 0.081 \\
\hline Recurrent angina & 104 (10.7) & 77 (7.8) & 0.059 & $41(9.4)$ & $29(5.3)$ & 0.127 \\
\hline
\end{tabular}

MACE, major adverse cardiac events; CAS, coronary artery spasm: PCI, percutaneous coronary intervention.

Table 2. Predictors of MACE up to 5 years using Multivariable Cox-Proportional Hazard Ratio Model Analysis (for Abstract no MP-13)

\begin{tabular}{|c|c|c|c|c|}
\hline \multirow[t]{2}{*}{ Variables } & \multicolumn{2}{|l|}{ Male } & \multicolumn{2}{|l|}{ Female } \\
\hline & $\begin{array}{l}\text { Hazard ratio } \\
95 \% \text { CI }\end{array}$ & $\mathrm{p}$ value & $\begin{array}{l}\text { Hazard ratio } \\
95 \% \text { CI }\end{array}$ & $\mathrm{p}$ value \\
\hline Age & 1.09 [1.02-1.17] & 0.006 & $0.98[0.90-1.08]$ & 0.767 \\
\hline Hypertension & $0.96[0.29-3.11]$ & 0.953 & $2.24[0.35-14.0]$ & 0.388 \\
\hline Uncontrolled blood pressure & 0.89 [0.27-2.87] & 0.847 & $1.20[0.21-6.84]$ & 0.837 \\
\hline Diabetes mellitus & $1.00[0.25-4.00]$ & 0.997 & $1.22[0.10-14.3]$ & 0.873 \\
\hline Dyslipidemia & $1.65[0.49-5.55]$ & 0.417 & $1.09[0.15-7.75]$ & 0.931 \\
\hline Current smokers & $2.18[0.65-7.23]$ & 0.201 & $7.77[0.52-115.4]$ & 0.136 \\
\hline Current alcohol drinkers & $0.80[0.24-2.59]$ & 0.710 & $1.11[0.10-11.7]$ & 0.927 \\
\hline Myocardial Bridge & $2.15[0.65-7.12]$ & 0.209 & $0.47[0.04-5.03]$ & 0.533 \\
\hline \multicolumn{5}{|l|}{ Insignificant stenosis } \\
\hline Mild (<30\%) & 1.08 [0.24-4.79] & 0.919 & $1.73[0.17-16.9]$ & 0.636 \\
\hline Mild (30-50\%) & $0.93[0.08-9.74]$ & 0.954 & $18.1[1.14-287.3]$ & 0.040 \\
\hline Moderate (50-70\%) & $6.15[1.12-33.5]$ & 0.036 & - & 0.994 \\
\hline
\end{tabular}

CI, Confidence interval; ARB, angiotensin receptor blockers; ACE inhibitors, angiotensin converting enzyme inhibitors.

the two groups (Table 1). In cox regression analysis for predicting five years MACE, old age (HR 1.09, $\mathrm{p}=0.006)$ and moderate stenosis $(50-70 \%$, HR 6.15, $\mathrm{P}=0.036)$ were significant predictors in male patients, and moderate stenosis $(30-50 \%$, HR 18.1, $\mathrm{p}=0.04)$ was significant predictor in female patients (Table 2). In cox regression analysis for predicting five years recurrent angina, moderate stenosis $(50-70 \%$, HR $2.83, \mathrm{p}=0.002)$ was significant predictor in male patients and myocardial bridge (HR 1.7, $\mathrm{p}=0.034)$ was significant predictor in female patients (Table 3).

Conclusion: In this study, GD was not found in five years long term clinical outcomes in CAS patients. However, in male and female patients, remaining moderate stenotic lesion is associated with five years MACE, and myocardial bridge in female patients and remaining moderate stenotic lesion are associated with recurrent angina during five years follow up. 
Abstracts: The Pulse of Asia 2018 Kyoto

Table 3. Predictors of Recurrent Angina up to 5 years using Multivariable Cox-Proportional Hazard Ratio Model Analysis (for Abstract no MP-13)

\begin{tabular}{|c|c|c|c|c|}
\hline \multirow[t]{2}{*}{ Variables } & \multicolumn{2}{|l|}{ Male } & \multicolumn{2}{|l|}{ Female } \\
\hline & $\begin{array}{l}\text { Hazard ratio } \\
{[95 \% \mathrm{CI}]}\end{array}$ & $\mathrm{p}$ value & $\begin{array}{l}\text { Hazard ratio } \\
{[95 \% \mathrm{CI}]}\end{array}$ & $\mathrm{p}$ value \\
\hline Age & 1.01 [0.99-1.03] & 0.232 & $1.00[0.98-1.02]$ & 0.728 \\
\hline Hypertension & $1.01[0.66-1.54]$ & 0.958 & $1.07[0.64-1.79]$ & 0.788 \\
\hline Uncontrolled blood pressure & $1.02[0.68-1.54]$ & 0.888 & $0.82[0.51-1.33]$ & 0.445 \\
\hline Diabetes mellitus & $0.80[0.47-1.36]$ & 0.420 & $0.76[0.39-1.49]$ & 0.433 \\
\hline Dyslipidemia & $1.07[0.67-1.71]$ & 0.749 & $1.67[0.99-2.82]$ & 0.053 \\
\hline Current smokers & 1.29 [0.86-1.95] & 0.212 & $1.30[0.39-4.28]$ & 0.658 \\
\hline Current alcohol drinkers & $0.70[0.46-1.06]$ & 0.093 & $0.57[0.25-1.27]$ & 0.174 \\
\hline Myocardial Bridge & $0.72[0.44-1.17]$ & 0.192 & $1.70[1.04-2.78]$ & 0.034 \\
\hline \multicolumn{5}{|l|}{ Insignificant stenosis } \\
\hline Mild $(<30 \%)$ & $0.93[0.58-1.49]$ & 0.770 & $0.73[0.43-1.25]$ & 0.263 \\
\hline Mild $(30-50 \%)$ & $1.06[0.49-2.28]$ & 0.867 & $1.43[0.66-3.09]$ & 0.353 \\
\hline Moderate (50-70\%) & $2.83[1.48-5.41]$ & 0.002 & $1.72[0.63-4.72]$ & 0.286 \\
\hline
\end{tabular}

CI, Confidence interval; ARB, angiotensin receptor blockers; ACE inhibitors, angiotensin converting enzyme.

\section{MP-14 \\ Relationship Between an Increased Urinary Albumin Excretion and Abnormal Electrocardiogram in the General Population}

Satoshi Konno Masanori Munakata

Division of Hypertension, Tohoku Rosai Hospital, Japan

Objective: Previous studies have reported relationships between an increased urinary albumin excretion and electrocardiogram (ECG) abnormalities, however, there are only a few studies conducted in Asian populations. The aim of this study was to examine whether elevated urinary albumin excretion is associated with the risk of atrial fibrillation and premature atrial contraction in the general Japanese population.

Design and Method: The study population consisted of 3,065 individuals from the general population who participated in an annual health check-up in 2016. We measured anthropometric parameters and sitting blood pressures after 5-min rest. A standard 12-lead ECG was recorded in the supine position. Fasting blood samples for lipids and glucose measurements, as well as morning spot urine samples for albumin-creatinine ratio were obtained. After excluding subjects with missing data, multi-variate logistic regression analysis was performed with the presence of atrial fibrillation and premature atrial contraction as dependent variables.

Results: Urinary albumin excretion (log-transformed) was significantly associated with the risk of atrial fibrillation (odds ratio: $2.187,95 \%$ confidence interval: 1.208-3.832) after controlling for known coronary risk factors. On the other hand, systolic blood pressure was a significant predictor for the presence of premature atrial contraction, although urinary albumin excretion was not.

Conclusion: An increased urinary albumin excretion may be associated with the risk of atrial fibrillation in the general Japanese population. 


\section{MP-15 \\ A Comparison of Brachial Artery Diameter with Flow-Mediated Vasodilation as Vascular Marker for Cardiovascular Risk Assessment: FMD-J Study \\ Tatsuya Maruhashi ${ }^{1}$, Masato Kajikawa ${ }^{2}$, Shinji Kishimoto ${ }^{1}$, Shogo Matsui ${ }^{1}$, Haruki Hashimoto ${ }^{1}$, Kensuke Noma 2,3, Hirofumi Tomiyama ${ }^{4}$, Akira Yamashina ${ }^{4}$, Yasuki Kihara ${ }^{1}$, Yukihito Higashi ${ }^{2,3}$ \\ ${ }^{1}$ Department of Cardiovascular Medicine, Graduate School of Biomedical and Health Sciences, Hiroshima University, ${ }^{2}$ Division of Regeneration and Medicine, Hiroshima University Hospital, ${ }^{3}$ Department of Cardiovascular Regeneration and Medicine, Research Institute for Radiation Biology and Medicine, ${ }^{4}$ Department of Cardiology, Tokyo Medical University}

Objective: Baseline brachial artery diameter has been reported to be a confounding factor of flow-mediated vasodilation (FMD). The purpose of this study was to investigate the relationships between baseline brachial artery diameter and cardiovascular risk factors and compare the diagnostic accuracy of baseline brachial artery diameter for subjects without cardiovascular risk factors and patients with cardiovascular disease (CVD) with that of FMD in men.

Method: We measured baseline brachial artery diameter and FMD in 5,695 male subjects. In addition, we retrospectively investigated the incidence of cardiovascular events using a separate population sample consisting of 440 male subjects to compare the accuracy of baseline brachial artery diameter for predicting cardiovascular events with that of FMD.

Results: Baseline brachial artery diameter and FMD significantly correlated with age, body mass index, systolic blood pressure, diastolic blood pressure, triglycerides, high-density lipoprotein cholesterol, and glucose as well as Framingham risk score. The prevalence of cardiovascular risk factors and CVD increased with increase in baseline brachial artery diameter and FMD. Area under the curve (AUC) value of the receiver operating characteristic (ROC) curve for baseline brachial artery diameter to diagnose subjects without cardiovascular risk factors $(0.59$ vs. $0.62, p=0.001)$ or patients with CVD $(0.58$ vs. $0.64, \mathrm{p}<0.001)$ was significantly lower than that for FMD. In the retrospective study, the AUC value of the ROC curve for baseline brachial artery diameter to predict first major cardiovascular events was significantly lower than that of FMD 0.50 vs. $0.62, \mathrm{p}=0.03$ ).

Conclusion: In men, baseline brachial artery diameter wasinferior to FMD for assessment of cardiovascular risk.

\section{MP-16}

\section{Comparison of Indices of Central and Peripheral Blood Pressure for Prediction of Cardiovascular Events in Short Term (25 Months) Follow-Up}

Junli Zuo ${ }^{1}$, Shaoli Chu², Guili Chang ${ }^{2}$, Isabella Tan ${ }^{3}$, Mark Butlin $^{3}$, Alberto Avolio 3

${ }^{1}$ Department of Hypertension, Geriatrics, Ruijin Hospital North, Shanghai Jiaotong University School of Medicine, Shanghai, China, ${ }^{2}$ Department of Hypertension, Ruijin Hospital North, Shanghai Jiaotong University School of Medicine, Shanghai, China, ${ }^{3}$ Department of Biomedical Sciences, Faculty of Medicine and Health Sciences, Macquarie University, Sydney, Australia

Objective: Prediction of haemodynamic parameters for cardiovascular events is conventionally assessed in follow-up studies of approximately five years or more. Since central aortic pressure may have stronger association with cardiac and cerebral function and may provide 
additional information compared to peripheral blood pressure, the aim of this study was to asses indices of central and peripheral pressure for prediction of cardiovascular events over a relatively shorter mean follow-up period of approximately two years.

Method: The longitudinal study cohort consisted of 668 patients (age $61 \pm 9$ years, 425 males, mean follow-up period $25 \pm 3.9$ months) was drawn from an initial cohort of 850 inpatients with primary hypertension. Noninvasive measuremenst were obtained for carotidfemoral pulse wave velocity, central aortic and brachial pressure, augmentation index (AIx), and pressure (AP) (SphygmoCor) and intima-media thickness (IMT).

Results: After adjusting for confounding factors, central systolic (cSP) and pulse pressure (cPP) showed higher hazard ratios $(\mathrm{HR} / 10 \mathrm{~mm})$ for cardiovascular events compared to peripheral pressure indices (pSP, pPP) at age $>60$ years (cSP: HR $=1.18$, pSP: HR $=1.17, \mathrm{p}=$ 0.034; $\mathrm{cPP}: \mathrm{HR}=1.28 \mathrm{pPP}, \mathrm{HR}=1.2 \mathrm{p}=0.019$ ), each SD increase in IMT and in AP entailed a 1.4 times higher risk of increased total events in older patients. For males, each SD increase in AP was associated with 1.36 times higher risk of increased total events. However, for females each SD decrease in central aortic AIx and AP was associated with 0.4 and 0.5 times lower risk of increased total and major cardiovascular events, respectively. This sex difference is most likely due to lack of age-related increase of AIx in females after age $>60$ years compared to males.

Conclusion: Central pressure improved prediction of $\mathrm{CV}$ events compared to peripheral pressure during a relatively short-term follow-up approximatey 2 years at age $60>$ years.

\section{MP-17 \\ Usefulness of Echocardiographic-Based Left Ventricular Hypertrophy in New Onset Atrial Fibrillation \\ Jiyeon Hong \\ Department of Cardiology, Hanil General Hospital, Korea}

Background: Left ventricular hypertrophy ( $\mathrm{LVH})$ is a risk factor for major cardiovascular events and mortality in patients with atrial fibrillation (AF).

Method: We evaluated echocardiographic (Echo) LVH prevalence, clinical factors and outcomes of patients with new onset non-valvular AF (NOAF). Among 35,611 patients with normal sinus rhythm who underwent Echo from August 2005 to June 2014, we enrolled 216 patients diagnosed with NOAF, who had completed data to diagnose LVH.

Results: We found that $34 \%$ of all NOAF patients had LVH. Patient with LVH had more female and hypertension (HT). LV ejection fraction, LV mass index by body surface area (LVMI), LV end diastolic dimension, left atrial dimeter (LAd), RV systolic pressure, E/e' and relative wall thickness (RWT) $\geq 0.42$ were associated with LVH. HT was more associated with LVH in men and RVT $\geq 0.42$ was more associated with LVH in women. During 10-year follow-up, patients with LVH received more percutaneous coronary intervention (PCI) after diagnosed with NOAF ( $\mathrm{p}<0.004)$. The relationship between LVH and follow up PCI was more prominent in women. Logistic regression analysis demonstrated that female $(\mathrm{p}<$ $0,000)$, HT $(p=0.006)$, increased LAd $(p=0.042)$ and LVMI $(p<0.000)$ were associated with LVH.

Conclusion: LVH was associated with NOAF. NOAF with LVH were related to female, HT, increased LAd and LVMI and associated with adverse cardiovascular outcomes such as higher incidence of PCI. 
Table 1. (for Abstract no MP-17)

\begin{tabular}{|c|c|c|c|c|}
\hline \multirow[t]{2}{*}{ Variable } & \multirow[t]{2}{*}{$\begin{array}{l}\text { All patients } \\
(\mathrm{n}=216)\end{array}$} & \multicolumn{2}{|c|}{$\begin{array}{l}\text { Left ventricular } \\
\text { hypertrophy }\end{array}$} & \multirow[t]{2}{*}{$P$ value } \\
\hline & & $\begin{array}{l}\text { Yes } \\
(n=75)\end{array}$ & $\begin{array}{l}\text { No } \\
(n=141)\end{array}$ & \\
\hline Age (years), mean+SD & $62.3+12.1$ & $67.0+9.3$ & $59.9+12.7$ & $<0.000$ \\
\hline Women (\%) & $103(47.7 \%)$ & $49(65.3 \%)$ & $54(38.3 \%)$ & $<0.000$ \\
\hline Body Mass Index $\left(\mathrm{Kg} / \mathrm{m}^{2}\right)$, mean+SD & $24.0+3.2$ & $24.7+3.4$ & $23.7+3.0$ & 0.018 \\
\hline Hypertension (\%) & $81(37.5 \%)$ & $37(49.3 \%)$ & $44(31.2 \%)$ & 0.009 \\
\hline Diabetes mellitus (\%) & $38(17.6 \%)$ & $18(24.0 \%)$ & $20(14.2 \%)$ & 0.071 \\
\hline Serum creatinine $(\mathrm{mg} / \mathrm{dL})$ & $1.2+1.8$ & $1.6+2.6$ & $0.9+1.2$ & 0.0171 \\
\hline \multicolumn{5}{|l|}{ Echocardiographic parameters } \\
\hline \multirow{2}{*}{\multicolumn{5}{|c|}{ Left ventricular mass index by body surface area $\left(\mathrm{g} / \mathrm{m}^{2}\right)$}} \\
\hline & & & & \\
\hline mean+SD & $102.7+33.6$ & $136.4+33.4$ & $84.7+14.2$ & $<0.000$ \\
\hline Relative Wall Thickness & $0.39+0.08$ & $0.41+0.10$ & $0.37+0.07$ & 0.003 \\
\hline \multicolumn{5}{|l|}{ Left ventricular inter End-diastolic dimension (mm), } \\
\hline mean+SD & $49.2+6.2$ & $52.5+6.7$ & $47.5+5.2$ & $<0.000$ \\
\hline Left atrial dimeter $(\mathrm{mm})$, mean+SD & $39.9+7.4$ & $43.2+7.7$ & $38.2+6.6$ & $<0.000$ \\
\hline $\mathrm{E} / \mathrm{e}^{\prime}$ & $11.4+6.0$ & $14.7+7.9$ & $9.7+3.7$ & $<0.000$ \\
\hline Right ventricular systolic pressure (mm Hg) & $32.0+9.4$ & $34.1+10.6$ & $30.9+8.6$ & 0.019 \\
\hline
\end{tabular}

MP-18

Renal Resistive Index Is an Early Predictor of Future Development of Anemia in Patients with Hypertension and Chronic Kidney Disease

Muneyoshi Tanimura, Kaoru Dohi, Ryuji Okamoto, Eitaro Fujii, Masaaki Ito

Department of Cardiology and Nephrology, Mie University Graduate School of Medicine, Japan

Objective: Chronic kidney disease (CKD) concerns a progression of anemia in various ways. Renal resistive index (RI) is known as a simple index of renal vascular resistance and also known to be associated with renal interstitial fibrosis, arteriosclerosis and renal function decline. We examined whether RI is associated with the presence and severity of anemia, and can predict its future development in patients with hypertension and CKD.

Method: We retrospectively examined 175 patients with hypertension (mean age $67 \pm$ 11 years, 32-85 years, 134 males) who underwent renal ultrasonography. Anemia was defined as a reduction in the concentration of hemoglobin $<13.0 \mathrm{~g} / \mathrm{dL}$ for men and $<12.0 \mathrm{~g} /$ $\mathrm{dL}$ for women. The renal RI was measured in the interlobar arteries.

Results: Anemia was present in $37 \%$ of men and $34 \%$ of women. The mean estimated glomerular filtration rate (eGFR) was $58 \pm 23 \mathrm{ml} / \mathrm{min} / 1.73 \mathrm{~m}^{2}$ (range: $16-168 \mathrm{ml} / \mathrm{min} / 1.73$ $\mathrm{m}^{2}$ ) and the mean renal RI was $0.70 \pm 0.09$ (range: 0.45-0.92). Proteinuria was present in $29 \%$ of patients. Both eGFR and RI correlated significantly with hemoglobin levels. In the multivariate analysis, RI was associated with hemoglobin levels independently of potential confounders including eGFR. During the follow-up period (1,325 \pm 822 , range: 6-3,314 days), age, renal RI and the presence of urine protein at baseline were independently correlated with the future development of anemia (age: odds ratio 1.03, 95\% CI 1.00-1.06; renal RI: odds ratio 2.15 , 95\% CI 1.26-3.66; urine protein: odds ratio 1.85, 95\% CI 1.18-2.91), and KaplanMeier curves demonstrated that patients with both renal RI $>0.68$ and positive proteinuria had higher incidence of the future development of anemia than other patients (Figure 1). 
Fig. 1. (for Abstract no MP-18).

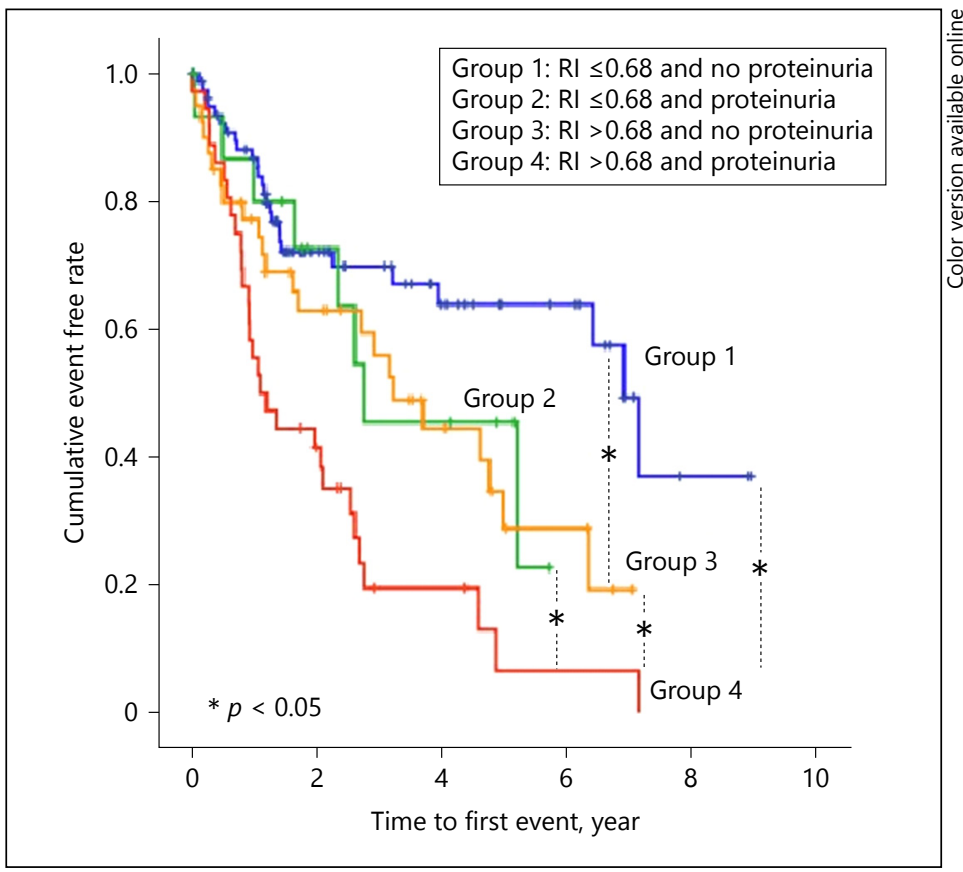

Conclusion: Measurement of renal RI can be useful for inferring its potential risk in early-stage CKD associated with hypertension.

MP-19

\section{Novel Measurement of Endothelial Function Assessed by Short Time Flow-Mediated Vasodilation: Comparison with Conventional Flow-Mediated Vasodilation}

Shogo Matsui ${ }^{1}$, Tatsuya Maruhashi, Masato Kajikawa ${ }^{2}$, Shinji Kishimoto ${ }^{1}$, Haruki Hashimoto ${ }^{1}$, Takayuki Hidaka', Kensuke Noma ${ }^{3}$, Yasuki Kihara ${ }^{1}$, Takeo Matsumoto ${ }^{4}$, Yukihito Higashi ${ }^{3}$

${ }^{1}$ Department of Cardiovascular Medicine, Graduate School of Biomedical Sciences, Hiroshima University, ${ }^{2}$ Division of Regeneration and Medicine, Medical Center for Translational and Clinical Research, Hiroshima University Hospital, ${ }^{3}$ Department of Cardiovascular Regeneration and Medicine, Research Institute for Radiation Biology and Medicine, Hiroshima University, ${ }^{4}$ Department of Electrical and Mechanical Engineering, Graduate School of Engineering, Nagoya University, Japan

Objective: Measurement of flow-mediated vasodilation (FMD) has been clinically established as a method for assessing endothelial dysfunction, leading to cardiovascular events. To shorten and simplify the measurement of FMD, we have developed a new device for assessment of endothelium-independent vasodilation, named short time FMD (stFMD). The objective of this study was to evaluate the validity of stFMD in comparison with conventional FMD.

Design and Method: We evaluated stFMD and conventional FMD in 82 subjects, including patients with atherosclerotic risk factors and cardiovascular disease (66 men and 16 women, $57 \pm 16$ yr) between December 2016 and February 2017. The measurements of stFMD and conventional FMD were carried out in random order. The observers were blind to the form of examination.

Results: In univariate regression analysis, stMD was significantly correlated with age $(\mathrm{r}=$ $-0.45, \mathrm{P}<0.001)$, systolic blood pressure $(\mathrm{r}=-0.42 \mathrm{P}<0.001)$, diastolic blood pressure $(\mathrm{r}=$ 
$-0.30, P=0.007)$, baseline brachial artery diameter $(r=-0.32, P=0.002)$ and conventional FMD $(r=0.76 \mathrm{P}<0.001)$ with consistent findings in conventional FMD. Bland-Altman plot analysis showed good agreements between stFMD and conventional FMD. Moreover, stFMD in the at risk group and that in the cardiovascular disease group were significantly lower than that in the no risk group ( $4.6 \pm 2.3 \%$ and $4.4 \pm 2.2 \%$ vs. $7.3 \pm 1.9 \%, p<0.001$, respectively). Optimal cutoff value of stFMD for diagnosing atherosclerotic patients is $7.0 \%$ (sensitivity, 71.0\%; specificity, $85.0 \%$ ).

Conclusion: Measurement of stFMD, a novel and simple method is useful for assessing endothelial function. stFMD may be suited for screening of atherosclerotic status in a large general population.

\section{MP-20 \\ Cuff Based Oscillometric Measurements of the Augmentation Index in Normotensive Japanese Individuals: Influence of Cuff Suitability on the Augmentation Index

\author{
Masakazu Obayashi', Shigeki Kobayashi', Michihiro Kohno3, Michiaki Kohno 1,2, \\ Tomoko Nakashima ${ }^{1}$, Masafumi Yano ${ }^{2}$ \\ ${ }^{1}$ Department of Cardiovascular Medicine, Sanyo-Onoda Municipal Hospital, ${ }^{2}$ Department of \\ Cardiovascular Medicine, Yamaguchi University School of Medicine, ${ }^{3}$ Kohno Clinic, Japan
}

Mobil-O-Graph is a cuff-based oscillometric device that can assess central augmentation index (AIx), and its usefulness in Europeans has been reported. However, the arm circumferences in Japanese are smaller than those in Europeans. We enrolled 110 normotensive volunteers (50 men; age range, 21-76 years). The left radial arterial waveform was recorded with the HEM-9000AI. The Mobil-0-Graph was placed on the left arms, and the arm circumferences were measured to allow the correct choice of cuff (two sizes available; $20-24 \mathrm{~cm}$ and 24-32 cm). We performed multiple regressions for central AIx and key variables in the MobilO-Graph. The arm circumferences (mean \pm SD) in men and women were $25.9 \pm 2.0 \mathrm{~cm}$ and $23.5 \pm 2.1 \mathrm{~cm}$, respectively. Both radial and central AIx in men were smaller than those in women. In men, central AIx using the larger cuff was significantly decreased compared with that using the smaller cuff $(14.5 \pm 6.3 \%$ vs $25.1 \pm 5.9 \%)$. In women, no difference was found in central AIx between both cuffs. No difference was observed in blood pressure between the two cuffs in both sexes. In multiple regression analysis, central AIx in men $\left(\mathrm{R}^{2}=0.549\right)$ was significantly associated with age $(\beta=0.185, P=0.002)$, height $(\beta=-0.380, P=0.029)$, and cuff size $(\mathrm{P}<0.001)$. Central AIx in women $\left(\mathrm{R}^{2}=0.222\right)$ tended to be associated with age $(\beta=$ $0.156, P=0.078)$, and was significantly associated with height $(\beta=-0.615, P=0.007)$ and heart rate $(\beta=-0.264, P=0.025)$. In conclusion, the brachial cuff-based waveform recordings were useful for Japanese normotensive individuals. However, the mean value of their arm circumference was closer to the lower limit of the arm circumference recommended for the larger cuff, and the measurement of lower central AIx using the larger cuff was less sensitive. This suggests that the influence of cuff suitability on central AIx would depend on brachial pressure waveform. 
MP-21

High Discordancy Between Unobserved Automated Office Blood Pressure and Ambulatory Blood Pressure in a Tertiary Hospital Cohort

Jiwon Seo, Chan Joo Lee, Donghoon Choi, Sungha Park

Cardiology Division, Severance Cardiovascular Hospital and Cardiovascular Research Institute, Yonsei University College of Medicine, Korea

Objective: Recently, automated office blood pressure (AOBP) has been shown to eliminate white coat effect and is more concordant with ambulatory blood pressure monitor (ABPM) and home BP measurements. The purpose of this study is to investigate the difference between blood pressure (BP) measured by AOBP compared with ABPM and to assess the prevalence of masked uncontrolled hypertension and white coat hypertension.

Design and Method: The study participants were recruited from a prospective cohort study (Cardiovascular and Metabolic Disease Etiology Research Center-High Risk Cohort [CMERC-HI]; clinicaltrials.gov: NCT02003781). 1,429 participants who had undergone both AOBP and ABPM within 7 days of each measurement were analyzed for this study.

Results: The 95\% limits of agreement between systolic AOBP and daytime ABPM systolic blood pressure (SBP) was -35.02 to $20.21 \mathrm{~mm} \mathrm{Hg}$ (mean difference $=-7.40$ ). The mean differences in quintiles of AOBP distributions increased with decreasing AOBP measurements [-17.7 \pm 11.2 (Q1, systolic AOBP <113), $-11.3 \pm 11.4$ (Q2, systolic AOBP 113-121), $-8.3 \pm$

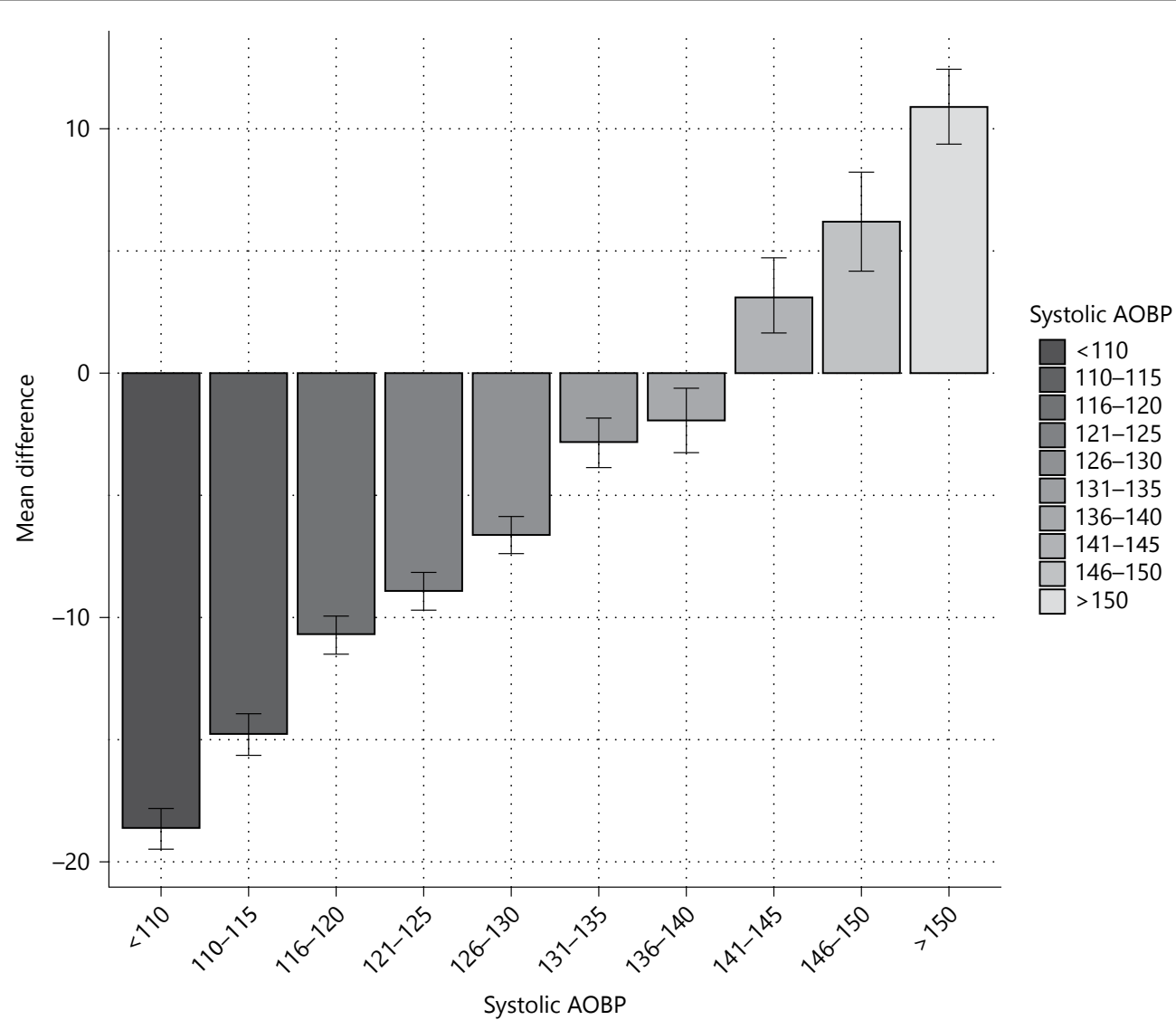

Fig. 1. (for Abstract no MP-21). 
10.7(Q3, systolic AOBP 121-128), $-4.4 \pm 12.1$ (Q4, systolic AOBP 128-137), $4.7 \pm 14.2$ (Q5, systolic AOBP >137)]. The prevalence of masked uncontrolled hypertension was $386(27 \%)$ and the prevalence of white coat effect was 105 (7.3\%). The prevalence of white coat effect in subjects with systolic AOBP $\geq 135 \mathrm{~mm} \mathrm{Hg}$ was 105 (31.5\%).

Conclusion: Lower range of systolic AOBP demonstrated large discrepancy with daytime ABPM SBP and high prevalence of masked hypertension. Status of BP control should be confirmed with out of office BP measurement.

\author{
MP-22 \\ The Predicting Value of WHtR to Atherosclerotic Cardiovascular Disease \\ Liang Zheng, Huimin Fan, Yuzhen Zhang \\ Shanghai East Hospital, China
}

Objective: To estimate the best cutoff value of waist-to-height ratio (WHtR) to atherosclerotic cardiovascular disease (ASCVD) and to compare the predicting value inWHtR, WC and BMI to ASCVD.

Method: Receiver operating characteristic (ROC) curve was performed to determine an optimal WHtR value to discriminate between individuals with intermediate and high 10-year estimated risk of ischaemic CVD fromlow-risk individuals. Then, the value with the highest sum of sensitivity and specificity (Youden index) was identifiedas the cut-off point. Multivariate logistic regression analyses were used to show the association of CVD risk factors with odds ratios (ORs) and 95\% confidence intervals (CI).

Results: The 3,950 participants were included in estimatingthe relationships between general and abdominal obesity indices with ASCVD, 21.5\% hadASCVD, 56.2\% had body mass index (BMI) $\geq 24 \mathrm{~kg} / \mathrm{m}^{2}, 50.1 \%$ had high waist circumference (WC). The prevalenceof abdominal obesitywas significantly greater in women $(81.2 \%)$ than in men $(71.8 \%, \mathrm{P}<$ 0.001 ). A significant increase in the prevalence of WHtR $\geq 0.50$ was found with increasing age in both genders, especially in women, reaching $91.7 \%$ in the women over 80 years. ROC analysis indicated that optimalWHtR cut-off value to predict the risk of ASCVDwas 0.53 with a prevalence of $55.8 \%$. Multivariate logistic regression analysesshowed that abdominal obesity indices of WHtR might be better markers than the general obesity index of BMI for the increased risk of ASCVD in elderly Chinese community population. The OR (95\% CI) of WHtR and BMI to ASCVD were 1.30 (1.01-1.43) and 1.21 (1.09-1.57), respectively.

Conclusion: The best cutoff value of WHtR predicting ASCVD was 0.53. Abdominal obesity and in particular a WHtR value above 0.53 was strongly associated with prevalent ASCVD and its risk factors with a higher value for odds ratio than WC and BMI in elderly Chinese subjects. 


\section{MP-23 \\ Comparison Between 24-Hour Brachial and Central Blood Pressures and Variability in their Associations with Target Organ Damage in Untreated Chinese}

Ying Wang Qianhui Guo, Dongyan Zhang, Yibang Cheng, Qifang Huang, Changsheng Sheng, Tingyan Xu, Jiguang Wang, Yan Li

Shanghai Institute of Hypertension, China

Background: 24-hour central blood pressure (BP) monitoring is now available for clinic use. However, its added value to 24-hour brachial BP monitoring is unclear. We therefore compared the associations between 24-hour brachial and central BPs and variability in their associations with target organ damage in untreated Chinese.

Method: Consecutive untreated patients referred for ambulatory BP monitoring to the Hypertension Outpatient Clinic of Ruijin Hospital were recruited from Sep 2013 to Dec 2015. The 24-hour brachial and central BPs were measured by oscillometric and automated devices (Mobil-O-Graph), left ventricular mass index and left ventricular diastolic function (E/e') by echocardiography, the urinary albumin-to-creatinine ratio (ACR) by immunobiochemical test, carotid intima-media thickness (IMT) by carotid ultrasonography, and aortic pulse wave velocity (PWV) by tonometry. Blood pressure variability (BPV) was assessed as standard deviation (SD), the coefficient of variation (CV), average real variability (ARV), the variability independent of the mean (VIM), and the difference between maximum and minimum BP (MMD). Effect sizes (standardized $\beta$ ) were computed and compared using multivariate regression models.

Results: The 24-hour brachial and central systolic/diastolic BP of the 315 participants (47.9\% males, mean age 53 years) averaged 126.2/82.3 $\mathrm{mm} \mathrm{Hg}$ and 118.3/83.6 $\mathrm{mm} \mathrm{Hg}$, respectively. All the brachial BPV indices of systolic BP were significantly $(\mathrm{P}<0.001)$ greater than their central counterparts. Multivariate regression analysis showed that both brachial and central systolic BP were significantly $(\mathrm{P} \leq 0.012)$ and similarly $(\mathrm{P} \leq$ 0.008) associated with E/e', PWV, log-transformed ACR, but the association with Carotid IMT was only observed for brachial systolic BP. However, there was no significant association of target organ measures with any BPV indices irrespective of measurement site $(\mathrm{P} \geq 0.07)$.

Conclusion: Both brachial and central BPs, but not BPV, were important determinants for target organ damage. Further research is needed to illustrate the added value of the 24-h central BP monitoring.

\section{MP-24 \\ A Case Report: Usefulness of Cibenzorin in Reducing Obstruction at Left Ventricular Outflow was Demonstrated by Pulse Wave Analysis \\ Masafumi Satoh Tomoyuki Kabutoya, Kazuomi Kario \\ Division of Cardiovascular Medicine, Jichi Medical University School of Medicine, Japan}

Objective: Cibenzoline is effective to reduce obstruction at left ventricular outflow due to hypertrophic cardiomyopathy. However, the detail assessment of pulse wave form before and after cibenzoline administration.

Methods: We evaluated pulse wave form before and after cibenzoline administration. The patient torelated $300 \mathrm{mg}$ three times a day. 
Results: Cibenzoline was effective in reduction of pressure gradient. Blood pressure was changed: $123.5 / 76.5 \mathrm{~mm} \mathrm{Hg}$ to $133.5 / 87.5 \mathrm{~mm} \mathrm{Hg}$. Upstroke time of right brachial artery was reduced $239 \mathrm{~ms}$ to $173 \mathrm{~ms}$, and that of left brachial artery was not changed ( 244 to $245 \mathrm{~ms}$ ).

Conclusions: Usefulness of cibenzorin in reducing obstruction at left ventricular outflow was demonstrated by pulse wave analysis.

\section{MP-25 \\ Comparative Effects of Valsartan Plus Either Cilnidipine or Hydrochlorothiazide on Nocturnal Home Blood Pressure in Patients with Morning Hypertension}

Takeshi Fujiwara ${ }^{1}$, Naoko Tomitani ${ }^{1}$, Hiroshi Kanegae ${ }^{2}$, Kazuomi Kario ${ }^{1}$

${ }^{1}$ Division of Cardiovascular Medicine, Jichi Medical University School of Medicine, Japan,

${ }^{2}$ Genki Plaza Medical Center for Health Care, Tokyo, Japan

Background: We tested our hypothesis that a valsartan/cilnidipine combination would reduce and optimize nocturnal home systolic blood pressure (HSBP), assessed by information and communication technology (ICT)-based home BP monitoring device, compared to a valsartan/hydrochlorothiazide combination in patients with morning hypertension, defined as morning SBP $\geq 135 \mathrm{~mm} \mathrm{Hg}$ or diastolic $\mathrm{BP} \geq 85 \mathrm{~mm} \mathrm{Hg}$.

Method: This study was a 8-week prospective, multicenter, randomized, open-label clinical trial conducted in Japan. Patients were asked to measure nocturnal HSBP over 3 nights prior to the study randomization and the end of treatment. Eligible patients were randomly allocated to either the valsartan/cilnidipine $(80 / 10 \mathrm{mg})$ or valsartan/hydrochlorothiazide (80/12.5 mg) combination groups.

Results: Forty-eight patients (mean 69.6 years, 39.6\% male) and fifty patients (mean 67.0 years, $46.0 \%$ male) were assessed in the valsartan/cilnidipine group and the valsartan/hydrochlorothiazide group, and their baseline nocturnal HSBP were $124.3 \pm 15.6$ and $125.8 \pm 15.2 \mathrm{~mm}$ $\mathrm{Hg}$, respectively ( $\mathrm{p}=0.597$ ). Although nocturnal HSBP was significantly reduced from baseline in both treatment groups, the valsartan/hydrochlorothiazide group significantly reduced nocturnal HSBP compared to the valsartan/cilnidipine group ( $-5.0 \mathrm{vs} .-10.0 \mathrm{~mm} \mathrm{Hg}, \mathrm{p}=0.035)$. However, the significant interaction between treatment groups concerning the baseline nocturnal HSBP level and the change in nocturnal HSBP was observed ( $p=0.047)$, which indicated that the valsartan/cilnidipine combination did not reduce nocturnal HSBP in patients with already controlled, while it could reduce nocturnal HSBP in patients with uncontrolled.

Conclusion: Valsartan/cilnidipine combination could reduce and optimize nocturnal HSBP levels more effectively than the valsartan/hydrochlorothiazide combination. 
Abstracts: The Pulse of Asia 2018 Kyoto

\section{MP-26 \\ Omega-3 Fatty Acid Improves Peripheral Artery Disease by Lowering Remnant-Like Lipoprotein Cholesterol in Hemodialysis Patients with Dyslipidemia: A Randomized Controlled Trial \\ Yusuke Kobayashi ${ }^{1}$, Tetsuya Fujikawa ${ }^{2}$, Tamio Iwamoto ${ }^{1}$, Nobuhito Hirawa ${ }^{3}$, Yoshiyuki Toya ${ }^{3}$, Kouichi Tamura ${ }^{3}$ \\ ${ }^{1}$ Division of Nephrology and Hypertension, Saiseikai Yokohamashi Nanbu Hospital, ${ }^{2}$ Center for Health Service Sciences, Yokohama National University, ${ }^{3}$ Department of Medical Science and Cardiorenal Medicine, Yokohama City University Graduate School of Medicine, Japan}

Objective: To evaluate the therapeutic effect of omega-3 fatty acid (EPA/DHA), which have potent anti-platelet and lipid lowering effects, on peripheral artery disease (PAD) in hemodialysis patients with dyslipidemia.

Design and Method: In this open-label, randomized parallel-group study, 38 hemodialysis patients with dyslipidemia were randomly assigned to receive either add-on of EPA/ DHA (1860 mg of EPA and $1500 \mathrm{mg}$ of DHA) with conventional therapy (EPA/DHA group: $\mathrm{n}=$ 19) or conventional therapy alone (control group: $n=19$ ) for 3 months. Remnant-like lipoprotein cholesterol (RLP-C) as an atherogenic lipid parameter, and ankle-brachial index (ABI) as an index of PAD were measured before and after the intervention. The changes of RLP-C and ABI in 3 months intervention were compared between the EPA/DHA group and the control group.

Results: After 3 months intervention, the mean RLP-C changed by $-3.25 \pm 3.15 \mathrm{mg} / \mathrm{dL}$ in the EPA/DHA group and by $0.44 \pm 2.53 \mathrm{mg} / \mathrm{dL}$ in the control group (between-group difference, 3.69; $\mathrm{p}<0.001$ ). The mean ABI changed by $0.07 \pm 0.11$ in the EPA/DHA group and by $-0.02 \pm$ 0.09 in the control group (between-group difference, $0.09 ; \mathrm{p}=0.007$ ). There was a significant negative correlation between the change in RLP-C and the change in ABI in the EPA/DHA group $(r=-0.475, p=0.040)$. In multiple regression analysis, the change in RLP-C was an independent determinant of the change in $\mathrm{ABI}$ after adjustment for age, gender and use of statin in EPA/DHA group ( $\mathrm{p}=0.042)$.

Conclusion: Even in hemodialysis patients with dyslipidemia undergoing conventional therapy such as use of statin, EPA/DHA add-on may be effective on improving PAD by lowering RLP-C.

\section{MP-27 \\ Severity of Obstructive Sleep Apnea Is Not Associated with Nocturnal BP Fluctuation in Elderly Hypertensive Patients Receiving Amlodipine \\ Satoko Nozato, Koichi Yamamoto, Kazuhiro Hongyo, Norihisa Ito, Hiromi Rakugi \\ Department of Geriatric and General Medicine, Osaka University Graduate School of Medicine, Japan}

Background: It was reported that obstructive sleep apnea (OSA) is associated with increased variability of nocturnal blood pressure (BP). Calcium channel blockers (CCBs) are shown to be superior to the other classes of antihypertensives in decreasing BP variability.

Aim: We investigated if severity of OSA is associated with nocturnal BP variability in elderly hypertensive patients treated with CCBs.

Method: This is a retrospective analysis of our previous prospective study targeting 23 hypertensive patients receiving amlodipine aged 65 years or older. Patients measured systolic 
and diastolic BPs, and pulse rate (PR) automatically during sleep at an interval of an hour once a week with an electronic sphygmomanometer (Medical Link, Omron) once a week. We calculated the coefficient of variation (CV=Standard deviation (SD) /mean value) in data from 4 consecutive measurements. All patients underwent home-based portable sleep study with SAS-2100 (Teijin Pharma limited).

Results: We classified patients into those with no-mild OSA and with moderate-severe OSA based on parameters of respiratory disturbances and desaturation using RDI and 3\% ODI, respectively. BMI was significantly higher in moderate-severe group than mild group in the categorization by RDI but not by ODI. There were no difference between groups in BPs, their variabilities, and PR in both categorization. The CV of PR in moderate-severe group was lower than mild group in the categorization by ODI but not by RDI. BMI was correlated with RDI and less with ODI ( $r=0.58$ and 0.50 , respectively). These parameters were not correlated with CV of BPs. CV in PR was significantly correlated with both RDI and ODI ( $r=0.42$ and 0.45 , respectively).

Conclusion: The severity of OSA correlated with variability of PR but not of BP in older patients receiving CCB. Our results would motivate future studies to determine if CCBs can suppress the influence of OSA on BP fluctuation during sleep.

\section{MP-28 \\ Fimasartan, an Angiotensin II Receptor Blocker, is Better Effective in Reducing Pulse Pressure with Similar Safety in Elderly Patients with Hypertension: K-MetS Study}

Jeong Bae Park ${ }^{6}$, Eun Joo Cho ${ }^{1}$, Ki Chul Sung ${ }^{2}$, Seok Min Kang ${ }^{3}$, Mi-Seung Shin ${ }^{4}$, Seung Jae Joo ${ }^{5}$

${ }^{1}$ Division of Cardiology, St. Paul's Hospital, Catholic University of Korea, Seoul, 'Division of Cardiology, Kangbuk Samsung Hospital, Sungkyunkwan University, Seoul, ${ }^{3}$ Division of Cardiology, Severance Cardiovascular Hospital, Yonsei University, Seoul, ${ }^{4}$ Division of Cardiology, Gachon University Gil Medical Center, Incheon, ${ }^{5}$ Division of Cardiology, Jeju National University Hospital, Jeju, ${ }^{6}$ Medicine, JB lab and clinic, Seoul, Korea

Guidelines recommend angiotensin II receptor blockers (ARBs) particularly in young patients with hypertension. But comparative safety and efficacy in elderly patients have not been established. This study was to determine the efficacy of ARB in elderly patients (age $\geq 60$ year-old) with hypertension using fimasartan, an ARB, by measuring clinic and home blood pressure (BP) and the safety and compared to non-elderly patients. In K-MetS study which is a nation-wide prospective study where patients enrolled from primary care clinic to tertiary care hospital, elderly patients (age $\geq 60$ year-old) who were treated for 1 year with fimasartan were recruited, and BPs measured at clinic and home were evaluated. A total of 2,363 elderly patients (46.9\% males, mean age 67.3 \pm 5.7 years) among 6,399 enrolled patients were evaluated. Overall, fimasartan in elderly patients reduced systolic and diastolic BPs (SBP and DBP) in clinic from $144.1 \pm 17.3$ to $127.7 \pm 12.9 \mathrm{~mm} \mathrm{Hg}$ and from $85.1 \pm 10.4$ to $76.8 \pm 8.4 \mathrm{~mm}$ $\mathrm{Hg}$ (all $\mathrm{p}<0.0001$ ) in 1 year. This efficacy of trend was similar at home. These findings were similarly seen in patients with non-elderly, but lower clinic DBP of $5.3 \mathrm{~mm} \mathrm{Hg}$ in elderly versus no-elderly patients at baseline showed less prominent drop of DBP through a year. However, pulse pressure, a better predictor of cardiovascular events in elderly, showed more prominent reduction of $-8.2 \pm 0.3 \mathrm{~mm} \mathrm{Hg}$ in elderly versus of $-7.0 \pm 0.2 \mathrm{~mm} \mathrm{Hg}$ (p value $<0.001$ ) in non-elderly after adjustment for sex, body mass index, diabetes mellitus, alcohol, and smoking. Adverse events were similar in elderly and non-elderly. In conclusion, a year treatment of fimasartan, an ARB, showed a better reduction of pulse pressure with similar 
efficacy of clinic and home BPs and similar safety in elderly patients with hypertension. Therefore, an ARB can be recommended regardless age or more recommended in high pulse pressure in elderly.

\section{MP-29 \\ The Gender Difference of the Effect of Cocoa-Derived Procynidin on Blood Pressure and Glucose Metabolism in Pre-Diabetic Subjects \\ Kazuki Shiina, Chisa Matsumoto, Masatsune Fujii, Shunsuke Komatsu, Hirofumi Tomiyama, Taishiro Chikamori \\ Department of Cardiology, Tokyo Medical University, Tokyo, Japan}

Objective: The present study was conducted to examine the effect of CACAO-derived antioxidant supplement on blood pressure, glucose/lipid metabolism and vascular function is subjects with early stage of abnormal glucose metabolisms, and also examined the gender difference of such effect by double-blind cross-over manner.

Method: In twenty men and women aged 40-69 years with pre-diabetic (HbA1c 5.8-6.5\%), CACAO procynidin $73.2 \mathrm{mg}$ /day or control (placebo) were prescribed for 4 weeks. Blood pressure, vascular functions (arterial stiffness and endothelial function), and other cardiovascular risk factors were measured at the beginning and the end of each intervention period.

Results: As a whole study subjects, no difference of any variables was observed between CACAO and CACAO-control interventions. As a sub-analysis, CACAO supplement reduced office diastolic blood pressure, homeostasis model assessment insulin resistance index and serum insulin levels in women, but these changes were not observed in men.

Conclusion: In pre-diabetic Japanese subjects, oxidative stress may affect the elevation of blood pressure and abnormalities in glucose/lipid metabolisms in postmenopausal women rather than men. In there, CACAO-derived antioxidant supplement may provide beneficial effect on these abnormalities.

\section{MP-30 \\ Case Report: Increasing Ambulatory Blood Pressure Variability and Pulse Pressure with Reverse Remodeling in Heart Failure}

Keisuke Narita ${ }^{1}$, , Satoshi Hoshide ${ }^{2}$, Kazuomi Kario ${ }^{2}$

${ }^{1}$ Department of cardiology, Karatsu Red Cross Hospital, Japan; ${ }^{2}$ Department of cardiology, Jichi Medical University, Tochigi, Japan

Blood pressure variability (BPV) and pulse pressure (PP) are generally decreased in heart failure with reduced left ventricular systolic function. Meanwhile, left ventricular reverse remodeling or improving systolic function increases BPV and PP.

We report a case of increasing BPV and PP with reverse remodeling in heart failure caused by hypertensive heart disease. A 30-year-old man had presented fatigue and weight gain lasting from one year ago. Although he had been indicated elevated blood pressure $(180 / 100 \mathrm{~mm} \mathrm{Hg})$ in an annual comprehensive medical examination since 5 years ago, he had not visited a health care facility. As a result of intensive examinations, he was diagnosed congestive heart failure caused from hypertensive heart disease. After initiating administration of beta-blocker, angiotensin-converting enzyme and others, left ventricular diameter 
was decreased and systolic function was improved. The increase of BPV and PP with reverse remodeling were observed by a repeat measuring of ambulatory blood pressure monitoring. Left ventricular reverse remodeling with using beta-blocker is often occurred in heart failure especially due to hypertensive heart disease. However, mechanisms of reverse remodeling in using beta-blocker or angiotensin-converting enzyme inhibitor (ACEI) is still unclear. We speculated a part of mechanisms of reverse remodeling in point of ambulatory blood pressure variability and pulse pressure in this case.

\section{MP-31 \\ Novel Hypertension Management Model of Care Resulting in Decreased Blood Pressure in a West African Population \\ Isaac Kofi Owusu ${ }^{1}$, Fred Adomako-Boateng ${ }^{2}$, Fred Kueffer ${ }^{3}$, Molly Guy $^{3}$, Chemuttaai Lang'at ${ }^{1}$, Teri Whitman ${ }^{3}$, Keith Holloman ${ }^{3}$, Douglas Hettrick ${ }^{3}$, K.O. Opare-Sem ${ }^{1}$ \\ ${ }^{1}$ Department of Medicine, Kwame Nkrumah University of Science and Technology, Kumasi, Ghana; ${ }^{2}$ Regioanl Health Directorate, Ghana Health Service, Kumasi, Ghana; ${ }^{3}$ Medtronic Inc., Minneapolis, MN, USA}

Background: A novel locally appropriate hypertension management model of care was developed and tested in the Republic of Ghana to address specific socioeconomic and cultural barriers to blood pressure control and to improve patient management efficiency and outcomes.

Methods: Patients with a history of hypertension were enrolled in the program and each agreed to visit a participating local community pharmacy at least once per week for 6 months for a BP check, symptom questions, and medication adherence monitoring. Weekly pharmacy visit data were manually logged into a mobile application that generated immediate personalized feedback. The patient's primary physician viewed real-time BP assessment data, and provided feedback directly to patients via text messages sent through the application and prescriptions sent directly to the pharmacy, as appropriate.

Results: Baseline BP of the 150 enrolled subjects ( $57 \pm 8$ years; $73 \%$ Female) was $135 \pm$ $18 / 84 \pm 10 \mathrm{~mm}$ Hg. During the 6-month voluntary program period, the number of scheduled office visits decreased by $60 \%$ compared to standard follow up schedules. Patient compliance with weekly BP assessments was $65 \%$ and 2,734 total pharmacy BP assessments were conducted. Despite fewer in clinic visits, population systolic blood pressure (SBP) dropped by $-4.8 \pm 18.2 \mathrm{~mm} \mathrm{Hg}(\mathrm{p}<0.01)$. The application directly referred 25 patients to a health facility for further evaluation. No deaths occurred, and six patients required inpatient hospitalization. Improvement in overall health awareness was reported in $82 \%$ of the patients and $95 \%$ indicated a desire to continue using the model of care in the future.

Conclusions: The multifaceted hypertension care model resulted in fewer clinic visits, high patient and clinician satisfaction and significant and sustained decreases in blood pressure without major safety concerns in this West African hypertensive population. 


\section{P-01 \\ Determinants of Inaccuracy of Validated Blood Pressure Monitors on Standardized Conditions}

Lei Lei

The Shanghai Institute of Hypertension, Ruijin Hospital, Shanghai Jiaotong University School of Medicine, China

Objective: In validation studies of blood pressure monitors, even validated devices may have a number of reading with a difference greater than 5,10 , or $15 \mathrm{~mm} \mathrm{Hg}$. In the present study, we investigated determinants of these inaccurate measurements using the data from validation studies at the study subject level.

Method: We pooled data from 16 validation studies according to the European society of Hypertension on protocols or the British Hypertension society protocol. Systolic and diastolic blood pressure was sequentially measured with the standard mercury sphygmomanometer and validated devices. Each study subject had 3 comparisons for systolic and diastolic blood pressures. In univariate and multivariate analysis, we considered sex, age, BMI, arm circumference and blood pressure level as possible determinants of blood pressure differences.

Results: Of the 628 study participants with 1,884 blood pressure comparisons, 418, 170 and 54 had at least one systolic or diastolic blood pressure difference greater than 5,10 and $15 \mathrm{~mm} \mathrm{Hg}$, respectively. If a blood pressure difference greater than $10 \mathrm{~mm} \mathrm{Hg}$ was chosen for analysis, the prevalence of inaccuracy was higher in those with an arm circumference $\geq 32 \mathrm{~cm}$ than those with an arm circumference $<32 \mathrm{~cm}(52.6 \%$ vs $31.0 \%, \mathrm{P}=0.048)$, and with higher systolic and diastolic blood pressure.(P for trend $\leq 0.007$ ) In multivariate analysis, the level of systolic and diastolic blood pressure remained statistically significant as determinants of inaccuracy readings.(for each $10 \mathrm{~mm} \mathrm{Hg}$ increment, $\mathrm{OR}=1.36$ and 1.28 for SBP and DBP, respectively).

Conclusion: Higher blood pressure level is a driving factor for the deviation of a validated device from standard mercury sphygmomanometer in blood pressure measurement.

\section{P-02 \\ Effects of the Valsartan/Amlodipine Combination and the Nifedipine GITS Monotherapy on Ambulatory Blood Pressure, Arterial Stiffness and Wave Reflection in Patients Inadequately Controlled with Monotherapy}

Shaokun Xu, Wei-Fang Zeng, Yan Li, Ji-Guang Wang

The Shanghai Institute of Hypertension, China

Objective: In a randomized controlled trial, we investigated effects of the valsartan/ amlodipine single-pill combination and the nifedipine GITS monotherapy on ambulatory blood pressure (BP), arterial stiffness and wave reflection in Chinese hypertensive patients who were inadequately controlled with monotherapy.

Method: In a subset of the study subjects enrolled in a 12-week, multicenter, open-label, active-controlled, parallel-group comparative study between valsartan/amlodipine single-pill combination and nifedipine GITS, we performed ambulatory BP monitoring $(n=150)$ and measurements of brachial-ankle pulse wave velocity ( $b-a P W V, n=118)$ and radial augmentation index (AI) ( $\mathrm{n}=70)$ at baseline and 12-week of follow-up. With the 24-h ambulatory BP, we also computed the ambulatory arterial stiffness index (AASI) as a measure of arterial stiffness. 
Results: At 12-week of follow-up, 24-h, daytime, nighttime mean systolic/diastolic BP decreased from 130.5/83.1, 134.7/86.3 and 122.8/77.7 $\mathrm{mm}$ Hg at baseline by 4.8/3.5, 3.8/2.6, and 7.2/5.2 $\mathrm{mm} \mathrm{Hg}$, respectively, in the valsartan/amlodipine group $(\mathrm{n}=75, \mathrm{P} \leq 0.046)$ and from 129.1/83.4, 132.5/86.0 and 121.4/78.0 $\mathrm{mm} \mathrm{Hg}$ at baseline by $2.7 / 1.8,1.7 / 1.1$, and $3.2 / 2.4 \mathrm{~mm} \mathrm{Hg}$ in the nifedipine GITS group $(\mathrm{n}=75, \mathrm{P} \leq 0.23)$. The least-square mean differences between the two groups were $2.1 / 1.7,2.0 / 1.5$, and $4.0 / 2.8 \mathrm{~mm} \mathrm{Hg}$, respectively $(\mathrm{P} \geq$ 0.06). AASI, however, did not change in either valsartan/amlodipine $(0.41 \mathrm{~m} / \mathrm{s}$ at baseline and change by $0.01 \mathrm{~m} / \mathrm{s}$ ) or nifedipine GITS ( 0.39 and $0.02 \mathrm{~m} / \mathrm{s}$, respectively). At week 12 , b-aPWV decreased from 16.7 and $15.7 \mathrm{~m} / \mathrm{s}$ at baseline by 1.6 and $0.7 \mathrm{~m} / \mathrm{s}$ in the valsartan/ amlodipine ( $\mathrm{n}=59, \mathrm{P}<0.0001)$ and nifedipine GITS groups $(\mathrm{n}=59, \mathrm{P}=0.067)$, respectively, while radial $\mathrm{AI}$ increased from $77.0 \%$ and $83.2 \%$ at baseline by $4.5 \%(\mathrm{P}=0.124)$ and $4.4 \%$ $(\mathrm{P}=0.046)$, respectively. The least square mean differences between the two groups did not reach statistical significance either for b-aPWV or AI.

Conclusion: Both the valsartan/amlodipine combination and nifedipine GITS reduced ambulatory BP and arterial stiffness, with a slightly more favorable effect for the combination therapy.

\section{P-03 \\ Role of Bisphenol a Exposure During Gestation and Lactation on the Emotional Behavior and the Content of Brain Neurotransmitters in Rat Offspring}

Arun Gandhi, R.K. Khanna

Department of Basic and Applied Biology, Vivekananda Global University, India

Background: Bisphenol A (BPA) is a well-known endocrine disruptor, and is recognized as having both estrogenic and anti-estrogenic effects, in addition to anti-androgenic and antithyroid effects. It has been also reported that BPA is transported to the fetus and neonatal infant through the placenta and breast milk. Objectives: The purpose of this study was to clarify the effects of BPA $(0,0.1$ and $1.0 \mathrm{ppm})$ exposure during the gestation and lactation stages on the behavior and the brain neurotransmitter content in rat offspring.

Method: The female rats were treated with 0.1 and $1.0 \mathrm{ppm}$ BPA in drinking water during gestational period ( 3 weeks) and lactational period ( 3 weeks), and the control group was given tap water throughout the experiment. In this study, six male offspring of each group were used. After open field-test and elevated plus-maze test (17-weeks old), the rats were killed with microwave exposure under anesthesia. The brains were dissected into five regions according to the method of Glowinski-Iversen, and each region was deprotenized. The content of neurotransmitters (acetylcholine) was measured by HPLC using electrochemical detector.

Results: In an open field-test, the group of BPAtreated offspring showed low nocturnal motor activity. In addition, this group tended to spend longer time on the open arm in the elevated plus-maze test. The content of acetylcholine in the striatum decreased. The content of noradrenalin and serotonin increased in the frontal cortex.

Conclusion: These results indicate that exposure to BPA during gestation and lactation may affect native behavior, and leads to an imbalance of neurotransmitters in the brain of offspring. 


\section{P-04}

Ethnic Difference in Carotid-Femoral Pulse Wave Velocity and Its Risk Profiles - Results from Two Community-Based Elderly Cohort Studies

Qian-Hui Guo ${ }^{1}$, Iram Faqir Muhammad ${ }^{2}$, Yan Borné2, Chang-Sheng Sheng1, Margaretha Persson 2, Ji-Guang Wang1', Gunnar Engström², Yan Li', Peter M. Nilsson ${ }^{2}$

${ }^{1}$ Shanghai Institute of Hypertension, ${ }^{2}$ Department of Clinical Sciences, Malmö, Lund University, Sweden

Objective: Carotid-femoral pulse wave velocity (cfPWV) and its risk factors may differ among various populations. However, few studies have directly compared the absolute value of cfPWV, and especially its associated risk profiles in different ethnicities.

Design and Method: The study population included 1,272 community-dwelling residents (age $75.0 \pm 6.5$ years, female 57.3\%) from Qingpu district, Shanghai, China, and 3,049 subjects (age $72.5 \pm 5.5$ years, female 60.4\%) from the Malmö Diet and Cancer Study re-examination, Malmö, Sweden. cfPWV was assessed by the SphygmoCor device in the same way in both cohorts.

Results: Multivariate linear regression analyses showed that cfPWV was positively associated with older age, body mass index (BMI), anti-hypertensive therapy, mean arterial pressure (MAP), heart rate, fasting glycose and triglycerides $(\mathrm{P}<0.05)$ in both populations. The effect size associated with age was greater in Swedish than in Chinese subjects $(\beta, 0.091$ vs $0.048, \mathrm{P}<0.001)$, while those associated with BMI (0.046 vs $0.008, \mathrm{P}<0.001)$, MAP $(0.079$ vs $0.067, \mathrm{P}=0.02)$, and heart rate $(0.060$ vs $0.039, \mathrm{P}=0.001)$ were larger in Chinese. No difference was observed in the effect size associated with fasting glucose and triglycerides. cfPWV was associated with male sex, total cholesterol and serum uric acid only in the Swedish subjects $(\mathrm{P} \leq 0.025)$. Multivariate-adjusted $\mathrm{cfPWV}$ was higher in Swedish than in Chinese (adjusted mean \pm standard error, $10.6 \pm 0.1$ vs $9.2 \pm 0.1 \mathrm{~m} / \mathrm{s}, \mathrm{P}<0.001$ ), and the ethnic difference remained significant in subgroups stratified by age, blood pressure, BMI and blood glucose.

Conclusion: Although Chinese and Swedish subjects shared similar major risk factors associated with arterial stiffness, ethnic differences existed in cfPWV and the strength of its associations with risk factors.

\section{P-05 \\ Systolic Blood Pressure Difference Between Arms and Chronic Kidney Disease in the Community Based 10-Year Cohort Study}

JongKwon Seo, Gwang-Sil Kim

Cardiology, Inje University Sanggye Paik Hospital, Korea

Objective: An increased inter-arm systolic blood pressure difference (IASBPD) is known to be related with the risk of cardiovascular (CV) events as well as peripheral vascular disease attributed to subclavian stenosis. Patients with chronic kidney disease is also known to have high cardiovascular risk. We investigate the association between the IASBPD and the incidence of chronic kidney disease (CKD) in a Korean population.

Design: This study conducted on 8,780 adults within a cohort of Korean population. We calculated the hazard ratios (HRs) for incident CKD using Cox proportional hazards models with potential confounding variables.

Method: IASBPD was defined as $\geq 15 \mathrm{~mm} \mathrm{Hg}$ using the average of initial and repeat blood pressure measurements obtained in both arms. [according to National Institute for Health and Clinical Excellence (NICE) guidelines] and CKD was defined as estimated glomerular filtration 
rate $<60 \mathrm{~mL} / \mathrm{min}$ per $1.73 \mathrm{~m}^{2}$. We assessed the predictable value of IASBPD for the incidence of CKD and also investigated for CV events including coronary heart disease and stroke.

Results: The prevalence of IASBPD was $6.6 \%$. The median duration of follow-up after first visit was 8.7 years. The incidence of the new development of CKD was higher in the IASBPD group than in the non-IASBPD group $(16.5 \%$ vs. $12.6 \%, p=0.013)$, and myocardial infarction was also more frequently noted in the IASBPD group than it was in the non-IASPBD group $(1.5 \%$ vs. $0.7 \%, \mathrm{p}=0.044)$. IASBPD proved to be a risk factor for incident CKD [Hazard ratio (HR) $=1.372,95 \%$ confidence interval (CI) 1.08-1.65, p-value 0.007] and the HR was also robust [HR 1.275, 95\% CI 1.03-1.58] after adjusting for potential confounders including age, hypertension, diabetes, obesity.

Conclusion: In this community-based cohort, increased IASBPD is associated with a significant risk predictor of the development of CKD in Korean general population.

\section{P-06 \\ Higher Cardioankle Vascular Index in 2nd Trimester of Pregnancy Predicts Maternal Complications}

Jeong Bae Park' ${ }^{1}$, Sua Kim²

${ }^{1} \mathrm{JB}$ lab and clinic, ${ }^{2}$ Department of Critical Care Medicine, Samsung Medical Center, Sungkyunkwan University, Korea

Objective: Cardiovascular adaptation differs significantly between normotension and hypertension in pregnancy and therefore, maternal outcomes differ. Because blood pressures (BP) are so variable during each trimester of pregnancy, we evaluated the usefulness of the measurement of BP-independent index of hemodynamics, cardioankle vascular index (CAVI) for the prediction of pregnancy-associated complication.

Design and Method: Diagnosis of hypertension was decided after delivery in hypertensive patients who referred to cardiology department and normotensive controls are agematched volunteers. Cardio-ankle vascular index (CAVI) and pulse wave velocity (PWV) was evaluated using VASERA VS-1000 (Fukuda-Denshi, Japan).

Table 1. Cardioankle vascular index and pulse wave velocity in each trimesters of pregnancy (for Abstract no P-06)

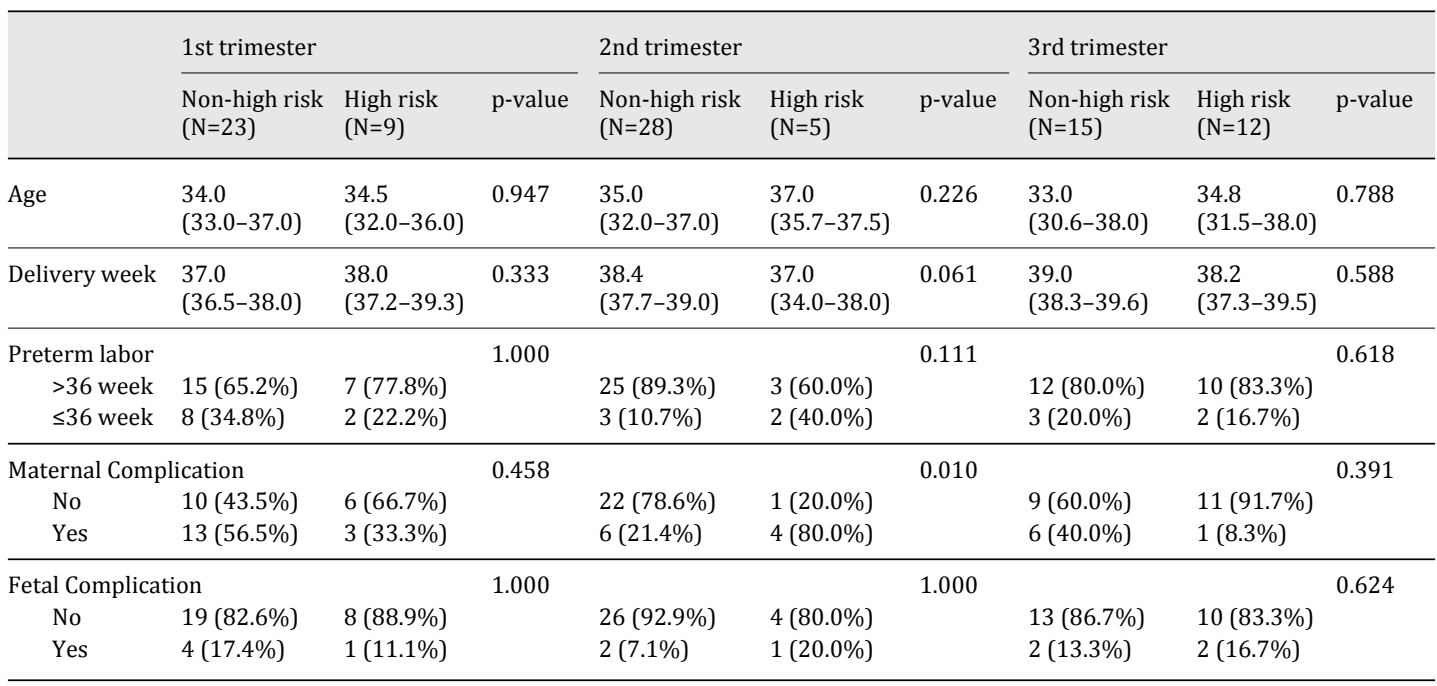


Results: Seventy-eight hypertensive patients (median age, 35.0 years (interquartile range, 32.0-37.0) and 41 normotensive subjects (median age, 34.0 years (interquartile range, 31.0-37.0)) were enrolled. The mean systolic and diastolic BPs were significantly higher in hypertensives than those of normotensives in most trimesters. CAVI was significantly higher in 1st and 3rd trimesters of hypertensives than normotensives, but PWV was higher only in the 1st trimester. According to cutoff values of SBP $130 \mathrm{~mm} \mathrm{Hg}$ and CAVI 6.0, high risk group (SBP and CAVI, $130 \mathrm{~mm} \mathrm{Hg}$ and 6.0 and higher) compared to non-high risk group showed significantly more maternal complications in 2nd trimester, but these findings were not found in 1st and 3rd trimesters.

Conclusion: Higher CAVI in the 2nd trimester during pregnancy, in which BPs are usually low and not different between normotensives and hypertensives, may help predict the maternal complications.

\section{P-07 \\ The Relationship Between Nocturnal Dipping Pattern and Morning Blood Pressure Surge in Hypertensive Patients}

Bae Keun Kim ${ }^{1}$, Jinho Shin'2, Sang Chil Lee ${ }^{3}$, Gi Soo Park ${ }^{3}$

${ }^{1}$ Division of cardiology, Department of Internal Medicine, Sung Ae General Hospital, ${ }^{2}$ Division of cardiology, Department of Internal Medicine, College of Medicine, Hanyang University, ${ }^{3}$ Division of cardiology, Department of Internal Medicine, Gwangmyeong Sung Ae General Hospital, Korea

Objective: The morning surge was considered as a physiological phenomenon in the morning. However, it may be associated with vascular damage and cardiovascular disease. Therefore, the aim of this study is to define the relationship between nocturnal dipping pattern and morning surge.

Method: Among ABPM database, data of 435 patients who underwent ABPM and were analyzed retrospectively. We classified the hyptensive patients into four groups using the following criteria: extreme dippers, a nocturnal SBP reduction rate of $\geq 20 \%$; dippers, a nocturnal SBP reduction rate of $10 \%$ to $<20 \%$; nondippers, a nocturnal SBP reduction rate of $0 \%$ to $<10 \%$; and risers, a nocturnal SBP reduction rate of $<0 \%$. Morning surge was quantified as the sleep-trough surge.

Results: Morning surge of hypertesive patients was $33.1 \pm 17.8 \mathrm{~mm} \mathrm{Hg}$. Percentage of extreme dippers, dippers, nondipper, and riser in hypertensive patients were $16.8 \%, 35.9 \%$, $32.4 \%$ and $14.9 \%$, respectively. In a multiple linear regression model for morning surge in hypertensive patients, dipping $(\beta=0.43, \mathrm{p}<0.001) 24$ hour systolic $\mathrm{BP}(\beta=0.16, \mathrm{p}<0.001)$ and antihypertensive medication $(\beta=7.03, p<0.001)$ were independently associated with morning surge. In a multiple logistic regression model for the high morning surge $(>41.4 \mathrm{~mm}$ $\mathrm{Hg}$; highest quartile) of hypertensive patients, odds ratio of extreme dipper was 3.01 (95\% CI: $1.46-6.10, p=0.002$ ), odds ratio of riser was 0.22 (95\% CI: $0.07-0.63, p=0.005$ ). 1.02 was odds ratio of 24 hour systolic BP (95\% CI: 1.00-1.04, $\mathrm{p}=0.017), 2.43$ was odds ratio of antihypertensive medication (95\% CI: 1.39-4.26, $\mathrm{p}=0.002$ ).

Conclusion: In hypertensive patients, abnormal dipping pattern, especially in riser group, was associated with morning surge. Maybe, abnormal dipping pattern and morning surge shared similar pathologic mechanism of vascular damage and cardiovascular disease. 


\section{P-08}

Supplementation of Vitamin D in Hypertensive Patients with CKD Stage 3 and Vitamin D-Deficiency Does Not Improve Arterial Stiffness

Enrique Rodilla Jose Antonio Costa, Jose Maria Pascual

Departamento de Medicina Interna, Hospital de Sagunto, Valencia, Spain

Objective: The role of supplementation of VitD in improving arterial stiffness is controversial. Aim of this study is to analyze if the supplementation of VitD in never before treated hypertensive patients with CKD stage 3 and Vitamin D-deficiency modifies arterial stiffness.

Method: Longitudinal study in the Hypertension Unit of a district hospital in 221 consecutive hypertensive patients with CKD stage 3 (CKD-EPI 30-60 ml/min), not treated before with VitD and with VitD-levels $<20 \mathrm{ng} / \mathrm{ml}$. Arterial stiffness was measured as Pulse Wave Velocity (PWV, m/s) and Augmentation Index (\%) in all the patients by brachial oscillometry (MOBIL-O-GRAPH ${ }^{\circledR}$, IEM, Stolberg, Germany). Oral Calcifediol (266 mircrog/month) was prescribed to all the patients. A follow-up visit was performed after 12 months.

Results: 126 (57\%) patients were women, 95 men (43\%). Mean age was 74.5 (SD \pm 9.5 ), years, renal function measured by CKD-EPI was $49.3 \mathrm{ml} / \mathrm{min}$ (SD \pm 7.2 ), BP averaged 130/66 $\mathrm{mm} \mathrm{Hg}$, mean number of antihypertensive drugs was 3.5. Mean levels of baseline VitD were $15.6 \mathrm{ng} / \mathrm{ml}(\mathrm{SD} \pm 6.6)$, mean $\mathrm{PWV}$ was $11.0 \mathrm{~m} / \mathrm{s}(\mathrm{SD} \pm 1.8)$. At follow-up after one year, mean levels of VitD in the whole group increased to 24.2 (DE \pm 11.5 ), PWV was $11.1 \mathrm{~m} / \mathrm{s}(\mathrm{DE} \pm 1.8$ ). $63.8 \%$ of the patients were still under supplementation with VitD at follow-up while $36.2 \%$ had stopped the treatment. In the former, VitD increased to $29.0 \mathrm{ng} / \mathrm{ml}$ versus $15.3 \mathrm{ng} / \mathrm{ml}$ in the latter. Although the significant difference between those patients who kept the treatment and those who stopped were highly significant, no significant change of PWV was found between the two groups at follow-up.

Conclusion: Correction of VitD-deficiency in hypertensive patients with CKD stage 3 is readily feasible, but in our study it did not translate into a significant change in vascular damage, measured as decrease of PWV, suggesting that the relationship between variables of arterial stiffness and VitD metabolism is not causal. 